\title{
On the wellposedness of some McKean models with moderated or singular diffusion coefficient
}

\author{
Mireille Bossy ${ }^{1}$ and Jean-François Jabir*2 \\ ${ }^{1}$ Université Côte d'Azur, Inria, France \\ ${ }^{2}$ Department of Statistics and Data Analysis, Higher School of Economics, Moscow, Russia
}

September 7, 2018

\begin{abstract}
We investigate the well-posedness problem related to two models of nonlinear McKean Stochastic Differential Equations with some local interaction in the diffusion term. First, we revisit the case of the McKean-Vlasov dynamics with moderate interaction, previously studied by Méléard and Jourdain in [16], under slightly weaker assumptions, by showing the existence and uniqueness of a weak solution using a Sobolev regularity framework instead of a Hölder one. Second, we study the construction of a Lagrangian Stochastic model endowed with a conditional McKean diffusion term in the velocity dynamics and a nondegenerate diffusion term in the position dynamics.
\end{abstract}

Key words: Weak-strong wellposedness problems; McKean-Vlasov models; Singular McKean diffusions.

\section{Introduction}

In this paper, we are interested in the wellposedness problem of some singular nonlinear McKean SDEs in the McKeanVlasov sense in $\mathbb{R}^{d}$, in the particular situation where the diffusion term carries the singular McKean nonlinear dependency. General form of nonlinear McKean SDEs is given by

$$
d X_{t}=b\left(X_{t}, \operatorname{Law}\left(X_{t}\right)\right) d t+\sigma\left(X_{t}, \operatorname{Law}\left(X_{t}\right)\right) d W_{t}, \quad \mu_{0}=\operatorname{Law}\left(X_{0}\right)
$$

where $\left(W_{t} ; t \geq 0\right)$ is a $\mathbb{R}^{d}$-valued standard Brownian motion, independent of $X_{0}$, and $\mu_{0}$ is a given probability measure on $\mathbb{R}^{d}$.

The class of singular McKean models we want to consider here are models for which the corresponding interacting particle system approximation (at least given formally, when the mean field limit is not yet established) gives rise to some singularity in the kernel function. In the context of the model (1.1), this means that for any $N \geq 2$, for any family of mollifiers $g_{\varepsilon}:=\varepsilon^{-d} g\left(\frac{x}{\varepsilon}\right)$ with parameter $\varepsilon>0$, the mapping

$$
x=\left(x^{0}, x^{1}, \ldots, x^{N}\right) \in \mathbb{R}^{(N+1) \times d} \mapsto b \text { or } \sigma\left(x^{0},\left(g_{\varepsilon} * \mu_{x}^{N}\right)\right) \in \mathbb{R}^{d}, \text { with } \mu_{x}^{N}=\frac{1}{N} \sum_{i=1}^{N} \delta_{x^{i}}
$$

attains its maximum norm on the subset $\bigcup_{i=1}^{N}\left\{x^{0}=x^{i}\right\}$ which tends to $+\infty$ as $\varepsilon$ tends to zero. Well known examples of McKean SDEs with singular drift kernel $b$ are those of the probabilistic interpretation of Burgers' equation (Sznitman [32]), the stochastic vortex method model for fluid flow (see e.g. Chorin [9], Méléard [25] among others), or

*The second author has been supported by the Russian Academic Excellence Project "5-100". 
more recently the probabilistic interpretation of the Keller Segel equation for chemotaxis modeling (see e.g. Fournier and Jourdain [19]).

More precisely, we are interested in the wellposedness of the following coupled processes $\left(X_{t}, Y_{t} ; t \geq 0\right)$ on a probability space $(\Omega, \mathcal{F}, \mathbb{P})$ satisfying the conditional McKean SDE

$$
\left\{\begin{array}{l}
X_{t}=X_{0}+\int_{0}^{t} b\left(X_{s}, Y_{s}\right) d s+\int_{0}^{t} \sigma\left(X_{s}\right) d B_{s} \\
Y_{t}=Y_{0}+\int_{0}^{t} \mathbb{E}_{\mathbb{P}}\left[\ell\left(Y_{s}\right) \mid X_{s}\right] d s+\int_{0}^{t} \mathbb{E}_{\mathbb{P}}\left[\gamma\left(Y_{s}\right) \mid X_{s}\right] d W_{s}
\end{array}\right.
$$

The initial condition $\left(X_{0}, Y_{0}\right)$ is distributed according to a given initial law $\mu_{0},\left(W_{t} ; t \geq 0\right)$ and $\left(B_{t} ; t \geq 0\right)$ are two independent $\mathbb{R}^{d}$ standard Brownian motions, $x \mapsto \sigma(x)$ is a $\mathbb{R}^{d} \times \mathbb{R}^{d}$ valued function. Before briefly describing our hypotheses on the coefficients of (1.3), let us make some comments on such models.

Our particular interest for the study of singular dynamics as (1.2) is motivated by the wellposedness problem related to the class of Lagrangian stochastic models for turbulent flows. This class of models have been introduced in the general framework of the statistical description of fluid motions and aimed to describe the main characteristic properties (position, velocity, ...) of a generic particle of a particular fluid flow. From the turbulent modeling viewpoint, such SDEs are known as Lagrangian "fluid-particle" models and are translation in a Lagrangian point of view (SDE) of some Eulerian PDE turbulence models (see e.g. Pope [29], [30], Durbin and Speziale [10]). These models involve a particular family of nonlinear McKean-Vlasov SDEs where the McKean nonlinearities are of conditional form. Such particular form of nonlinearity models the influence of the macroscopic components of the flow on the particle motion. In some of our recent works, [4], [5], we have studied toy-version models of conditional McKean SDEs where the singularity is concentrated in the drift term. From a mathematical viewpoint, the wellposedness results obtained in [4],[5] are still far from covering the complexity of a meaningful 'fluid-particle' model, as such Lagrangian models contain conditional McKean nonlinearity in both drift and diffusion components. In this paper, we focus on singular McKean diffusive characteristic that motivates our interest in new wellposedness results in that direction.

In [4],[5] and for the construction of (numerical) approximation (we refer to Bossy et al. [6], [7] for some numerical description cases and experiments), we analyze the SDE (1.2) in the framework of an apriori existing density $\rho_{t}(x, y) d x d y=\mathbb{P}\left(X_{t} \in d x, Y_{t} \in d y\right)$. The model (1.2) thus becomes

$$
\left\{\begin{array}{l}
X_{t}=X_{0}+\int_{0}^{t} b\left(X_{s}, Y_{s}\right) d s+\int_{0}^{t} \sigma\left(X_{s}\right) d B_{s} \\
Y_{t}=Y_{0}+\int_{0}^{t} \Lambda\left[X_{s} ; \rho_{s}\right] d s+\int_{0}^{t} \Gamma\left[X_{s} ; \rho_{s}\right] d W_{s}
\end{array}\right.
$$

with $\Lambda$ and $\Gamma$ defined, for $(x, f)$ in $\mathbb{R}^{d} \times L^{1}\left(\mathbb{R}^{d} \times \mathbb{R}^{d}\right)$, as

$$
\Lambda[x ; f]=\frac{\int_{\mathbb{R}^{d}} \ell(y) f(x, y) d y}{\int_{\mathbb{R}^{d}} f(x, y) d y} \mathbb{1}_{\left\{\int_{\mathbb{R}^{d}} f(x, y) d y \neq 0\right\}} \quad \text { and } \quad \Gamma[x ; f]=\frac{\int_{\mathbb{R}^{d}} \gamma(y) f(x, y) d y}{\int_{\mathbb{R}^{d}} f(x, y) d y} \mathbb{1}_{\left\{\int_{\mathbb{R}^{d}} f(x, y) d y \neq 0\right\}}
$$

In comparison, our wellposedness result for the solution of (1.2), presented in Section 3, uses a $L^{2}(\Omega)$-fixed point construction and a suitable Girsanov transformation that relies on the strong ellipticity assumption on $\sigma$. Essentially, our working hypotheses will be to assume boundedness and Lipschitz continuity of $b, \sigma, \ell$ and $\gamma$ for the wellposedness of a weak solution to (1.2), and some $L^{p}$ density condition on the initial distribution and a uniform elliptic property on $\gamma$ to handle pathwise uniqueness. At all time $t$, the time-marginal distributions $\operatorname{Law}\left(X_{t}, Y_{t}\right)$ of this strong solution further admit a density function $\rho_{t}$, and so our constructed solution to (1.2) is also solution to (1.3).

In the context of complex flow modeling, we would like to emphasise that a targeted form of (1.2) is a coupled position-velocity $\left(X_{t}, U_{t} ; 0 \leq t \leq T\right)$ kinetic process with degenerate diffusion in the $X$-component together with a 
linear drift $b(x, y)=y$ :

$$
\left\{\begin{array}{l}
X_{t}=X_{0}+\int_{0}^{t} U_{s} d s \\
U_{t}=Y_{0}+\int_{0}^{t} \mathbb{E}_{\mathbb{P}}\left[\ell\left(U_{s}\right) \mid X_{s}\right] d s+\int_{0}^{t} \mathbb{E}_{\mathbb{P}}\left[\gamma\left(U_{s}\right) \mid X_{s}\right] d W_{s}
\end{array}\right.
$$

But unbounded drift case, degenerate diffusion and singular McKean kernel are a mixture of difficulties that are quite hard to overcome jointly.

For future works, to overcome the strong ellipticity assumption on $\sigma$ in (1.2), we further investigate some weaker characterisation method based on mild-equation formulation as in [5]. In Section 2, we present a step further in that direction, applying such technique for our second study case on moderated McKean local diffusion equation:

$$
\left\{\begin{array}{l}
X_{t}=X_{0}+\int_{0}^{t} \sigma\left(u\left(s, X_{s}\right)\right) d W_{s}, \quad 0 \leq t \leq T, \\
d \operatorname{Law}\left(X_{t}\right)=u(t, x) d x \text { with } u \in L^{\infty}\left((0, T) \times \mathbb{R}^{d}\right) \cap L^{2}\left((0, T) \times \mathbb{R}^{d}\right), \\
u(0, x)=u_{0}(x), x \in \mathbb{R}^{d}, \text { where } u_{0} \text { is a given probability density function on } \mathbb{R}^{d},
\end{array}\right.
$$

for any arbitrary time horizon $0<T<\infty$. Nevertheless, our existence proof based on approximation method needs some strict monotonicity assumption which still coincides with the strong ellipticity in the one dimensional framework. In [16], Jourdain and Méléard studied a moderately interacting model such as (1.4), extending a previous work from Oelschläger [28] on a moderately interacting model, where both the drift and diffusion coefficients depend locally on the time marginal densities of the law of the solution that are supposed to be smooth enough. Whenever the nonlinearity is reduced to the diffusion part, the model in [16] reduces to:

$$
\left\{\begin{array}{l}
X_{t}=X_{0}+\int_{0}^{t} \sigma\left(p\left(s, X_{s}\right)\right) d W_{s}, \quad 0 \leq t \leq T \\
d \operatorname{Law}\left(X_{t}\right)=p(t, x) d x \text { with } p \in \mathcal{C}_{b}^{1,2}\left([0, T] \times \mathbb{R}^{d}\right), \\
p(0, \cdot) \text { is a given probability density that belongs in the Hölder space } H^{2+\alpha}\left(\mathbb{R}^{d}\right) \text { with } 0<\alpha<1 .
\end{array}\right.
$$

In Section 2, we prove the wellposdness of a strong solution of (1.4), mainly replacing the condition $p \in \mathcal{C}_{b}^{1,2}([0, T] \times$ $\left.\mathbb{R}^{d}\right)$ by $p \in L^{\infty}\left((0, T) \times \mathbb{R}^{d}\right) \cap L^{2}\left((0, T) \times \mathbb{R}^{d}\right)$ (replacing the strong ellipticity condition on $\sigma$ needed for the equation (1.5) by a strict monotonicity condition). Our proof is focused on the simple case where the diffusion component is given by $\sigma(r) I_{d}$ for a scalar function $\sigma:[0, \infty) \rightarrow[0, \infty)$. Extensions to further multidimensional diffusion component are discussed at the end of Section 2.

Our main results are Theorem 2.3 in Section 2 which states the strong wellposedness for the moderated McKean local diffusion equation (1.4), and Theorem 3.3 in Section 3 for the strong wellposedness of conditional McKean SDEs (1.3). In the two cases, we obtained weak uniqueness of the solution with slighty weaker conditions.

We end this introductory section with a short review of results and approaches from the literature for SDEs with McKean diffusion term, in order give some insights to the reader with the two particular cases that we are addressing in this paper.

\section{Review of some wellposedness results for nonlinear SDEs with McKean diffusion term}

We consider McKean-Vlasov SDEs of the following specific form in $\mathbb{R}^{d}$,

$$
X_{t}=X_{0}+\int_{0}^{t} \sigma\left(X_{s}, \operatorname{Law}\left(X_{s}\right)\right) d W_{s}, 0 \leq t \leq T,
$$

up to a (possibly infinite) horizon time $T$.

Under the assumptions that $\mathbb{E}\left[\left|X_{0}\right|^{p}\right]<\infty, 1 \leq p<\infty$, and $\sigma$ is continuous on $\mathbb{R}^{d} \times \mathcal{P}_{p}\left(\mathbb{R}^{d}\right)$ for $\mathcal{P}_{p}\left(\mathbb{R}^{d}\right)$ being the space of probability measures with $p$-th finite moments, Funaki [13] showed the existence, on any arbitrary time 
interval, of a weak solution to (1.6) in terms of a martingale problem. Uniqueness of the solution to the martingale problem holds under the assumption that

$$
\|\sigma(x, \mu)-\sigma(y, \nu)\| \leq C|x-y|+\kappa\left(\mathcal{W}_{p}(\mu, \nu)\right),
$$

where $\mathcal{W}_{p}$ is the Wasserstein distance endowed with the cost function $|x-y|^{p}$, and $\kappa:[0, \infty) \rightarrow[0, \infty)$ is a strictly increasing function such that $\kappa(0)=0$ and $\lim _{\epsilon \rightarrow 0^{+}} \int_{\epsilon}^{\infty} 1 / \kappa^{2}(\sqrt{r}) d r=\infty$. Oelschläger [27] considered the analog situation where $\sigma$ is bounded and Lipschitz for the metric

$$
\|\mu-\nu\|=\sup \left\{\int f(x)(\mu(d x)-\nu(d x)): \max _{x \in \mathbb{R}^{d}}|f(x)| \leq 1 \text { and }\|f\|_{\text {Lip }}:=\sup _{x \neq y} \frac{|f(x)-f(y)|}{|x-y|} \leq 1\right\},
$$

and proved the existence of a solution in law, as well as a weak propagation of chaos result for the related stochastic particle system. Both cases include the particular situation when the interaction kernel has the form: $\sigma(x, \mu)=$ $\int_{\mathbb{R}^{d}} \sigma(x, y) \mu(d y)$. Moreover, in this framework, Méléard [24] showed, through a fixed point argument in the space $\left(\mathcal{P}_{2}\left(\mathcal{C}\left([0, T] ; \mathbb{R}^{d}\right)\right), W_{2}\right)$, that whenever, $\sigma: \mathbb{R}^{d} \times \mathbb{R}^{d} \rightarrow \mathbb{R}^{d \times d}$ is Lipschitz continuous w.r.t. the two variables, the pathwise wellposedness and strong-pathwise propagation of chaos holds for the related stochastic particle system.

Jourdain and Méléard [16] extended the work of Oelschläger [28] on the moderately interacting drift term model and prove the wellposedness of (1.5) with the following assumptions:

○ $p(0, x)=p_{0}(x)$ where $p_{0}$ belongs to the Hölder space $H^{2+\alpha}\left(\mathbb{R}^{d}\right)$ with $0<\alpha<1$;

○ $\sigma: r \in \mathbb{R} \mapsto \sigma(r) \in \mathbb{R}^{d \times d}$ is a Lipschitz function, $\mathcal{C}^{3}$ on $\mathbb{R}$, with values in the space of symmetric non-negative matrices $d \times d$;

- Strong ellipticity holds for $\sigma$ : there exists $m_{\sigma}>0$ such that $\forall x \in \mathbb{R}^{d}, \forall r \in \mathbb{R}, \quad x^{*} \sigma(r) x \geq m_{\sigma}|x|^{2}$;

○ Non negativity holds for the diffusion matrix leading to the Fokker-Planck equation written on divergence form:

$$
\forall x \in \mathbb{R}^{d}, \forall r \in \mathbb{R}, \quad x^{*}\left(\left(\sigma \sigma^{*}\right)^{\prime}(r) r+\left(\sigma \sigma^{*}\right)(r)\right) x \geq 0 ;
$$

(This latest assumption is used to derive the uniqueness from the Fokker-Planck equation related to (1.5), written in divergence form:

$$
\left\{\begin{array}{l}
\frac{\partial p}{\partial t}=\sum_{i=1}^{d} \frac{\partial}{\partial x_{i}}\left(\frac{1}{2} \sum_{j=1}^{d}\left(\left(\sigma \sigma^{*}\right)_{i j}^{\prime}(p) p+\left(\sigma \sigma^{*}\right)_{i j}(p)\right) \frac{\partial p}{\partial x_{j}}\right) \text { on }(0, T) \times \mathbb{R}^{d} \\
p(0, x)=p_{0}(x), x \in \mathbb{R}^{d}
\end{array}\right.
$$

from maximum principle argument.)

○ Strong ellipticity holds on the leading matrix: there exists $m_{\text {div }}>0$ such that

$$
\forall x \in \mathbb{R}^{d}, \forall r \in \mathbb{R}, \quad x^{*}\left(\left(\sigma \sigma^{*}\right)^{\prime}(r) r+\left(\sigma \sigma^{*}\right)(r)\right) x \geq m_{\mathrm{div}}|x|^{2} .
$$

(With this additional assumption, the Cauchy problem (1.7) has a solution in $H^{1+\frac{\alpha}{2}, 2+\alpha}\left(\mathbb{R}^{d}\right)$, and the nonlinear SDE admits a unique strong solution.)

Kohatsu-Higa and Ogawa in [20] considered nonlinear McKean-Vlasov dynamic in convolution form

$$
X_{t}=X_{0}+\int_{0}^{t} A\left(X_{s}, \sigma * \operatorname{Law}\left(X_{s}\right)\right) d W_{s} .
$$

Assuming that $A$ and $\sigma$ are Lipschitz with at most linear growth, they prove the wellposedness of a strong solution and particle-time discrete approximation. 
Jourdain and Reygner in [17] considered particular cases of scalar equation related to, and around, porus media equation which correspond to the case of equation (1.8) with $\sigma(x)=\mathbb{1}_{\{x \geq 0\}}$ and $A(x, u)=A(u)>0$. The case $A(u) \geq 0$ is also studied using the limit of a reordered particle system.

Recently, Mishura and Veretennikov in [26] consider a model of the form

$$
d X_{t}=\sigma\left[t, X_{t} ; \operatorname{Law}\left(X_{t}\right)\right] d W_{t}, X_{0} \sim \mu_{0}
$$

where

$$
\sigma[t, x ; \mu]=\int \sigma(t, x, y) \mu(d y)
$$

for $\sigma:(0, \infty) \times \mathbb{R}^{d} \times \mathbb{R}^{d} \rightarrow \mathbb{R}^{d \times d}$. Assuming that $X_{0}$ has finite fourth order moments, $(x, y) \mapsto \sigma(t, x, y)$ has linear growth, uniformly in $t$, and $(t, x, y) \mapsto \sigma(t, x, y) \sigma^{*}(t, x, y)$ is uniformly strongly elliptic, the SDE admits at least one weak solution.

We end this review by mentioning some recent works in the direction of the wellposedness of the following system of SDE

$$
\left\{\begin{array}{l}
\frac{d S_{t}}{S_{t}}=r d t+\frac{a\left(Y_{t}\right)}{\sqrt{\mathbb{E}\left[a^{2}\left(Y_{t}\right) \mid S_{t}\right]}} \sigma_{\text {Dup }}\left(t, S_{t}\right) S_{t} d W_{t}, \\
d Y_{t}=\alpha\left(t, Y_{t}\right) d B_{t}+\xi(t) d t .
\end{array}\right.
$$

Such models arise in mathematical finance for the calibration of local and stochastic volatility models, and where $\sigma_{\text {Dup }}(t, y)$ is the Dupire's local volatility function (see Gyongy [15]). We emphasise that a major difference with the model (1.2) is in the conditioning variable which is the coupled variable $X_{t}$ in our case and the unknown $S_{t}$ in the volatility calibration model. This particular case generates different and yet hard difficulties compared to (1.2). The existence of a local-in-time solution to the Fokker-Planck equation associated to this model has been established by Abergel and Tachet in [1], while Jourdain and Zhou[18] recently obtained a first global-in-time wellposedness result in the case when $Y$ is a (constant in time) discrete valued random variable.

\section{Some notations}

Hereafter, $\mathcal{C}$ denotes the space of continuous functions equipped with the uniform norm $\|f\|_{\infty}=\max |f|$ and $\mathcal{C}^{k}$ denotes the space of $k$ times continuously differentiable functions. $\mathcal{C}_{c}$ and $\mathcal{C}_{c}^{k}$ respectively refer to the corresponding compactly supported subsets. For $m \geq 1$, and $1 \leq p \leq \infty, L^{p}\left(\mathbb{R}^{m}\right)$ denotes the Lebesgue space of all Borel (measurable) functions $f: \mathbb{R}^{m} \rightarrow \mathbb{R}$ such that $\|f\|_{L^{p}\left(\mathbb{R}^{m}\right)}^{p}:=\int|f(z)|^{p} d z<\infty$, and $W^{1, p}\left(\mathbb{R}^{m}\right)$ denotes the Sobolev space

$$
W^{1, p}\left(\mathbb{R}^{m}\right)=\left\{f \in L^{p}\left(\mathbb{R}^{m}\right) \text { s.t. }\left\|\nabla_{z} f\right\|_{L^{p}\left(\mathbb{R}^{m}\right)}<\infty\right\}
$$

equipped with the norm $\|f\|_{W^{1, p}\left(\mathbb{R}^{m}\right)}:=\|f\|_{L^{p}\left(\mathbb{R}^{m}\right)}+\left\|\nabla_{z} f\right\|_{L^{p}\left(\mathbb{R}^{m}\right)}$. As usual, $H^{1}\left(\mathbb{R}^{m}\right)$ stands for the particular space $W^{1,2}\left(\mathbb{R}^{m}\right)$. For $1 \leq q \leq \infty, L^{q}\left((0, T) ; W^{1, p}\left(\mathbb{R}^{m}\right)\right)$ denotes the space of Borel functions $f$ defined on $(0, T) \times$ $\mathbb{R}^{m}$ such that

$$
\|f\|_{L^{q}\left((0, T) ; W^{1, p}\left(\mathbb{R}^{m}\right)\right)}:=\|f\|_{L^{q}\left((0, T) ; L^{1, p}\left(\mathbb{R}^{m}\right)\right)}+\|\nabla f\|_{L^{q}\left((0, T) ; L^{1, p}\left(\mathbb{R}^{m}\right)\right)}
$$

is finite. Finally, the index loc will refer to local integrability property, namely $f$ belongs in $L_{l o c}^{p}$ if for all $0 \leq R<\infty$, $f \mathbb{1}_{\{B(0, R)\}}$ is in $L^{p}\left(\mathbb{R}^{m}\right)$.

\section{The moderated McKean local diffusion equation revisited}

In this section, we consider the wellposedness problem, up to an arbitrary finite horizon time $T>0$, for the following SDE:

$$
\begin{aligned}
& X_{t}=X_{0}+\int_{0}^{t} \sigma\left(u\left(s, X_{s}\right)\right) d W_{s}, \quad 0 \leq t \leq T, \\
& d \operatorname{Law}\left(X_{t}\right)=u(t, x) d x \text { where } u \text { belongs in } L^{\infty}\left((0, T) \times \mathbb{R}^{d}\right) \cap L^{2}\left((0, T) \times \mathbb{R}^{d}\right), \\
& u(0, x)=u_{0}(x), x \in \mathbb{R}^{d}, \text { where } u_{0} \text { is a given probability density function. }
\end{aligned}
$$


For the sake of simplicity, from now on, we restrict ourselves to the case of a diffusion matrix $\sigma$ mainly diagonal; that is $\sigma(r)=\sigma(r) I_{d}$ for $\sigma: \mathbb{R} \rightarrow \mathbb{R}$ and $I_{d}$ the identity $d \times d$ matrix. Extensions to more general diffusion matrices will be discussed at the end of this section (see Subsection 2.4).

Let us further point out that the notion of solution to (2.1) is intentionally restricted to the class of solutions satisfying (2.1b). We consider the class of solutions of continuous processes satisfying (2.1a) and whose time-marginal distributions admit a representant in $L^{2}\left(\mathbb{R}^{d}\right)$ for a.e. $0 \leq t \leq T$, and in $L^{\infty}\left((0, T) \times \mathbb{R}^{d}\right)$. The choice of working with this particular class is mainly motivated by the use of comparison principles and energy estimates techniques (see e.g. Evans [11] and Vasquez [34]) for the time-marginal distributions solution to the Fokker-Planck equation related to (2.1). Energy estimates will enable us to construct a suitable approximation to (2.1) and also to deduce the uniqueness of the marginal distributions $u(t), 0 \leq t \leq T$. We emphasise that the divergence form for the Fokker-Planck equation makes appear as a coefficient the map $r \mapsto \alpha(r)$ defined as

$$
\alpha(r):=\left(\sigma^{2}(r) r\right)^{\prime}=2 \sigma^{\prime}(r) \sigma(r) r+\sigma^{2}(r), \text { for } r \in \mathbb{R}^{+},
$$

which our main hypothesis is based on.

Remark: In the case when $\sigma$ and $\alpha$ are bounded, our proof arguments and subsequent wellposedness results can be extended to the class of solutions to (2.1a) satisfying $u \in L^{2}\left((0, T) \times \mathbb{R}^{d}\right)$ in place of $(2.1 \mathrm{~b})$.

Throughout this section, Equation (2.1) is considered under the following set of assumptions:

\section{Hypothesis 2.1.}

$\left(\boldsymbol{A}_{0}\right) u_{0}$ is a probability density function in $L^{1}\left(\mathbb{R}^{d}\right) \cap L^{\infty}\left(\mathbb{R}^{d}\right)$ such that $\int_{\mathbb{R}^{d}}|x|^{2} u_{0}(x) d x<\infty$.

$\left(\boldsymbol{A}_{1}\right)$ The map $r \mapsto \sigma(r) \in \mathbb{R}$ is continuously differentiable on $\mathbb{R}^{+}$.

$\left(\boldsymbol{A}_{2}\right)$ The map $r \mapsto \alpha(r)$ is continuous on $\mathbb{R}^{+}$, and there exists some constant $\eta>0$ such that

$$
\alpha(r) \geq \eta>0, \forall r \geq 0
$$
$\sigma^{2}$ :

Assumption $\left(A_{2}\right)$ ensures that $\sigma$ is in $\mathcal{C}^{1}([0, \infty))$ and implies a classical assumption on the uniform positivity of

$$
\sigma^{2}(r) \geq \eta, \forall r \geq 0
$$

which further implies the uniform ellipticity of $\sigma^{2} I_{d}$. Yet, most of the time, we will also make use of the following assumption which, together with a monotonic property of $\alpha$ (see Theorem 2.3 and Proposition 2.10 below), allows possible degeneracy of the diffusion $\sigma$ at point $r=0$ :

\section{Hypothesis 2.2.}

(A $\boldsymbol{A}_{2}$-weakened) The map $r \mapsto \alpha(r)$ is continuous on $\mathbb{R}^{+}$, and

$$
\alpha(r) \geq 0, \forall r \geq 0 \text {. }
$$

The main result of this section is the wellposedness for equation (2.1) given by the following theorem:

Theorem 2.3. Under Hypothesis 2.1, there exists a unique strong solution to (2.1). Uniqueness in law holds true under $\left(A_{0}\right),\left(A_{1}\right),\left(A_{2}\right.$-weakened) with the additional hypothesis that $r \mapsto \alpha(r)$ is strictly increasing.

Main ingredients and steps of the proof. The rest of this section is devoted to our proof of Theorem 2.3 that relies on the following three main ingredients:

1. An appropriate form of $\varepsilon$-nondegenerate approximation of the diffusion $\sigma$. In a first step, we show the wellposedness of a family of $\varepsilon$-approximation $\left\{\left(u^{\varepsilon}(t), X_{t}^{\varepsilon} ; 0 \leq t \leq T\right), \varepsilon>0\right\}$ to (2.1) where $\sigma$ is replaced by $\sigma_{\varepsilon}$ defined by

$$
\sigma_{\varepsilon}^{2}(r)=\sigma^{2}(r)+\varepsilon, \forall r \geq 0 .
$$

Notice that (2.3) produces a suitable approximation of the map $r \mapsto \alpha(r)$ by

$$
\alpha_{\varepsilon}(r)=\left(\sigma_{\varepsilon}^{2}(r) r\right)^{\prime}=\left(\sigma^{2}(r) r\right)^{\prime}+\varepsilon=\alpha(r)+\varepsilon, \forall r \geq 0
$$


2. The construction of $\left(u^{\varepsilon}(t), X_{t}^{\varepsilon} ; 0 \leq t \leq T\right)$ is then obtained from a preliminary existence result of a $L^{\infty}((0, T) \times$ $\left.\mathbb{R}^{d}\right) \cap L^{2}\left((0, T) ; H^{1}\left(\mathbb{R}^{d}\right)\right)$-weak solution to the related Fokker-Planck equation, and some uniform energy estimates w.r.t $\varepsilon$ on this solution. Such estimates allow to deduce successively the relative compactness of $\left\{u^{\varepsilon}, \varepsilon>0\right\}$ in $L^{2}\left((0, T) ; H^{1}\left(\mathbb{R}^{d}\right)\right)$ and of $\left\{\operatorname{Law}\left(X_{t}^{\varepsilon} ; 0 \leq t \leq T\right), \varepsilon>0\right\}$ in $\mathcal{P}\left(\mathcal{C}\left([0, T] ; \mathbb{R}^{d}\right)\right)$. The corresponding limits of converging subsequences are then shown to jfbe a solution of the martingale problem related to (2.1). This main step is stated in Proposition 2.8 below.

3. Uniqueness in law is obtained from a mild-form equation in $L^{2}\left((0, T) \times \mathbb{R}^{d}\right)$ derived from the Fokker-Planck equation in Proposition 2.10. The mild approach used here allows us to get rid of the strong ellipticity hypothesis for $\sigma$, at least at point 0 . The weak uniqueness result is then obtained under $\left(A_{2}\right)$, but also under $\left(A_{2}\right.$-weakened) with the adding of the strict monotonicity for $\alpha$. Uniqueness in the pathwise sense is stated in Proposition 2.12.

\subsection{Nondegenerate approximation of (2.1)}

In this section, we construct a solution to the SDE

$$
\begin{aligned}
& X_{t}^{\varepsilon}=X_{0}+\int_{0}^{t} \sqrt{\sigma_{\varepsilon}^{2}\left(u^{\varepsilon}\left(s, X_{s}\right)\right)} d W_{s}, \quad 0 \leq t \leq T, \\
& d \operatorname{Law}\left(X_{t}^{\varepsilon}\right)=u^{\varepsilon}(t, x) d x, \text { with } u^{\varepsilon} \in L^{\infty}\left((0, T) \times \mathbb{R}^{d}\right) \cap L^{2}\left((0, T) \times \mathbb{R}^{d}\right), \\
& u^{\varepsilon}(0, x)=u_{0}(x), x \in \mathbb{R}^{d},
\end{aligned}
$$

where $\sigma_{\varepsilon}^{2}(r)=\sigma^{2}(r)+\varepsilon, \forall r \geq 0$, and we show some appropriate density estimates for the marginal densities $u^{\varepsilon}(t)$. Defined as such, the diffusion coefficient $\sigma_{\varepsilon}$ still satisfies $\left(A_{1}\right)$. Our existence proof is mainly deduced from a PDE analysis of the smoothed Fokker-Planck equation related to (2.5):

$$
\left\{\begin{array}{l}
\frac{\partial u^{\varepsilon}}{\partial t}-\frac{1}{2} \triangle_{x}\left(\sigma_{\varepsilon}^{2}\left(u^{\varepsilon}\right) u^{\varepsilon}\right)=0, \text { on }(0, T) \times \mathbb{R}^{d}, \\
u^{\varepsilon}(0, x)=u_{0}(x), x \in \mathbb{R}^{d} .
\end{array}\right.
$$

We proceed for the existence of a solution to (2.6), first by exhibiting the existence of a weak solution of a linearized version of (2.6), and next by a fixed point argument we deduce the existence. These results are given in the following two lemmas:

Lemma 2.4. Assume $\left(A_{0}\right),\left(A_{1}\right)$ and $\left(A_{2}\right.$-weakened). Let $v=v(t, x)$ be a non-negative given function belonging to $L^{\infty}\left((0, T) \times \mathbb{R}^{d}\right) \cap L^{2}\left((0, T) \times \mathbb{R}^{d}\right)$. Then there exists $\bar{u}^{\varepsilon} \in L^{2}\left((0, T) ; H^{1}\left(\mathbb{R}^{d}\right)\right) \cap \mathcal{C}\left([0, T] ; L^{2}\left(\mathbb{R}^{d}\right)\right)$ such that, for all $f \in \mathcal{C}_{c}^{1,2}\left([0, T] \times \mathbb{R}^{d}\right)$, for all $0 \leq T_{0} \leq T$,

$$
\begin{aligned}
\int_{\mathbb{R}^{d}} \bar{u}^{\varepsilon}\left(T_{0}, x\right) f\left(T_{0}, x\right) d x-\int_{\mathbb{R}^{d}} u_{0}(x) f(0, x) d x \\
\quad=\int_{\left(0, T_{0}\right) \times \mathbb{R}^{d}} \bar{u}^{\varepsilon}(t, x) \partial_{t} f(t, x)-\frac{1}{2} \alpha_{\varepsilon}(v(t, x)) \nabla_{x} \bar{u}^{\varepsilon}(t, x) \cdot \nabla_{x} f(t, x) d t d x,
\end{aligned}
$$

for $\alpha_{\varepsilon}$ defined as in (2.4). In addition, $\bar{u}^{\varepsilon}$ has nonnegative values a.e. on $(0, T) \times \mathbb{R}^{d}$,

$$
\left\|\bar{u}^{\varepsilon}\right\|_{L^{\infty}\left((0, T) \times \mathbb{R}^{d}\right)} \leq\left\|u_{0}\right\|_{L^{\infty}\left(\mathbb{R}^{d}\right)},
$$

and

$$
\max _{t \in[0, T]}\left\|\bar{u}^{\varepsilon}(t)\right\|_{L^{2}\left(\mathbb{R}^{d}\right)}^{2}+\varepsilon \int_{0}^{T}\left\|\nabla_{x} \bar{u}^{\varepsilon}(t)\right\|_{L^{2}\left(\mathbb{R}^{d}\right)}^{2} d t \leq\left\|u_{0}\right\|_{L^{2}\left(\mathbb{R}^{d}\right)}^{2} .
$$


For the nonlinear PDE (2.6), we extend the notion of a $L^{2}\left((0, T) ; H^{1}\left(\mathbb{R}^{d}\right)\right)$-weak solution, stated in this lemma 2.4, as a function $u^{\varepsilon} \in L^{2}\left((0, T) ; H^{1}\left(\mathbb{R}^{d}\right)\right)$ such that: for all $0<T_{0} \leq T$, and $f \in \mathcal{C}_{c}^{\infty}\left(\left[0, T_{0}\right] \times \mathbb{R}^{d}\right)$,

$$
\begin{aligned}
\int_{\mathbb{R}^{d}} u^{\varepsilon}\left(T_{0}, x\right) f\left(T_{0}, x\right) d x-\int_{\mathbb{R}^{d}} u_{0}(x) f(0, x) d x \\
\quad=\iint_{\left(0, T_{0}\right) \times \mathbb{R}^{d}} u^{\varepsilon}(t, x) \partial_{t} f(t, x) d t d x+\int_{\left(0, T_{0}\right) \times \mathbb{R}^{d}} \frac{1}{2} \sigma_{\varepsilon}^{2}\left(u^{\varepsilon}(t, x)\right) u^{\varepsilon}(t, x) \triangle_{x} f(t, x) d t d x .
\end{aligned}
$$

Lemma 2.5. Assuming that $\left(A_{0}\right),\left(A_{1}\right)$ and $\left(A_{2}\right.$-weakened) hold, the nonlinear PDE (2.6) admits a unique nonnegative $\mathcal{C}\left([0, T] ; L^{2}\left(\mathbb{R}^{d}\right)\right) \cap L^{2}\left((0, T) ; H^{1}\left(\mathbb{R}^{d}\right)\right)$-weak solution $u^{\varepsilon}$. This solution is uniformly bounded with

$$
\left\|u^{\varepsilon}\right\|_{L^{\infty}\left((0, T) \times \mathbb{R}^{d}\right)} \leq\left\|u_{0}\right\|_{L^{\infty}\left(\mathbb{R}^{d}\right)},
$$

and satisfies the energy inequality:

$$
\sup _{t \in[0, T]}\left\|u^{\varepsilon}(t)\right\|_{L^{2}\left(\mathbb{R}^{d}\right)}^{2}+\varepsilon \int_{0}^{T}\left\|\nabla_{x} u^{\varepsilon}(t)\right\|_{L^{2}\left(\mathbb{R}^{d}\right)}^{2} d t \leq\left\|u_{0}\right\|_{L^{2}\left(\mathbb{R}^{d}\right)}^{2} .
$$

In addition, we have, for all $0 \leq T_{0} \leq T$,

$$
\int_{\mathbb{R}^{d}} \Psi_{\varepsilon}\left(u^{\varepsilon}\left(T_{0}, x\right)\right) d x+\frac{1}{2} \int_{\left(0, T_{0}\right) \times \mathbb{R}^{d}}\left|\nabla_{x} \Phi_{\varepsilon}\left(u^{\varepsilon}(t, x)\right)\right|^{2} d t d x=\int_{\mathbb{R}^{d}} \Psi_{\varepsilon}\left(u_{0}(x)\right) d x,
$$

for $\Phi_{\varepsilon}(r)=\sigma_{\varepsilon}^{2}(r) r$ and $\Psi_{\varepsilon}(r)=\int_{0}^{r} \Phi_{\varepsilon}(\theta) d \theta$.

Now the existence of a weak solution to (2.5) first could be classically reformulated into a martingale problem. Owing to the boundedness of $(t, x) \mapsto \sigma\left(u^{\epsilon}(t, x)\right)$, this gives (see Theorem 2.6, in Figalli [12]) the following result.

Proposition 2.6. Under $\left(A_{0}\right),\left(A_{1}\right)$ and $\left(A_{2}\right.$-weakened), there exists a unique weak solution $\left(X_{t}^{\varepsilon} ; 0 \leq t \leq T\right)$ to (2.5) such that the time marginal densities are given by $\left(u^{\varepsilon}(t) ; 0 \leq t \leq T\right)$ from Lemma 2.5 .

\subsubsection{Proof of Lemma 2.4}

For any $v \in L^{2}\left((0, T) ; H^{1}\left(\mathbb{R}^{d}\right)\right)$, the identity (2.4) ensures that, for a.e. $t \in(0, T)$, the bilinear mapping

$$
\left(u_{1}, u_{2}\right) \mapsto \mathcal{L}_{t}\left(u_{1}, u_{2}\right)=\int_{\mathbb{R}^{d}} \frac{1}{2} \alpha_{\varepsilon}(v(t, x))\left(\nabla_{x} u_{1}(x) \cdot \nabla_{x} u_{2}(x)\right) d x
$$

is continuous on $H^{1}\left(\mathbb{R}^{d}\right) \times H^{1}\left(\mathbb{R}^{d}\right)$, since $\mathcal{L}_{t}\left(u_{1}, u_{2}\right) \leq \frac{1}{2}\left(\sup _{0 \leq r \leq\|v\|_{L} \infty} \alpha_{\varepsilon}(r)\right)\left\|\nabla_{x} u_{1}\right\|_{L^{2}\left(\mathbb{R}^{d}\right)}\left\|\nabla_{x} u_{2}\right\|_{L^{2}\left(\mathbb{R}^{d}\right)}$. Moreover, since $\mathcal{L}_{t}(u, u) \geq \frac{\epsilon}{2}\left\|\nabla_{x} u\right\|_{L^{2}\left(\mathbb{R}^{d}\right)}^{2}, \mathcal{L}_{t}$ satisfies the hypothesis of Theorem 1.1 in Lions [23, Chapter 4] : $\mathcal{L}_{t}(u, u)+\frac{\epsilon}{2}\left\|u^{2}\right\|_{L^{2}\left(\mathbb{R}^{d}\right)} \geq \frac{\epsilon}{2}\left\|u^{2}\right\|_{H^{1}\left(\mathbb{R}^{d}\right)}$ for all $u \in H^{1}\left(\mathbb{R}^{d}\right)$. Applying Theorem 1.1 and Lemma 1.1 in [23], we deduce the existence of a solution $\bar{u}^{\varepsilon} \in L^{2}\left((0, T) ; H^{1}\left(\mathbb{R}^{d}\right)\right)$ to

$$
\begin{gathered}
\int_{\mathbb{R}^{d}} \bar{u}^{\varepsilon}\left(T_{0}, x\right) f\left(T_{0}, x\right) d x-\int_{\mathbb{R}^{d}} u_{0}(x) f(0, x) d x \\
=\int_{\left(0, T_{0}\right) \times \mathbb{R}^{d}} \bar{u}^{\varepsilon}(t, x) \frac{\partial f}{\partial t}(t, x)-\frac{1}{2} \alpha_{\varepsilon}(v(t, x))\left(\nabla_{x} \bar{u}^{\varepsilon}(t, x) \cdot \nabla_{x} f(t, x)\right) d t d x, \\
\forall 0 \leq T_{0} \leq T, \forall f \in \mathcal{C}_{c}^{\infty}\left(\left[0, T_{0}\right] \times \mathbb{R}^{d}\right) .
\end{gathered}
$$

The property of $u^{\varepsilon} \in \mathcal{C}\left([0, T] ; L^{2}\left(\mathbb{R}^{d}\right)\right)$ can be proved in the same way as in [23], Theorem 2.1, Chapter 4 .

The energy estimate (2.9) is obtained by adapting some arguments of Ladyženskaja et al. [22], p. 141-142. For $0 \leq T_{0} \leq T$, for $h>0$ such that $T_{0}+h \leq T$, and $\eta_{h} \in \mathcal{C}_{c}^{\infty}\left(\left(-h, T_{0}\right) \times \mathbb{R}^{d}\right)$ such that $\eta_{h}(t, x)=0$ whenever $t \leq 0$ or $t \geq T_{0}-h$, we define

$$
\phi(t, x)=\frac{1}{h} \int_{t-h}^{t} \eta_{h}(s, x) d s=\int_{-1}^{0} \eta_{h}(t+s h, x) d s .
$$


Plugging $\phi$ as a test function into the weak formulation (2.7) gives

$$
\begin{aligned}
& u_{0}(x) f(0, x) d x \\
& 0=\int_{\left(0, T_{0}\right) \times \mathbb{R}^{d}} \bar{u}^{\varepsilon}(t, x) \partial_{t} \phi(t, x)-\frac{1}{2} \alpha_{\varepsilon}(v(t, x))\left(\nabla_{x} \bar{u}^{\varepsilon}(t, x) \cdot \nabla_{x} \phi(t, x)\right) d t d x .
\end{aligned}
$$

Since $\partial_{t} \phi(t, x)=\frac{1}{h}\left(\eta_{h}(t, x)-\eta_{h}(t-h, x)\right)$,

$$
\begin{aligned}
\int_{\left(0, T_{0}\right) \times \mathbb{R}^{d}} \bar{u}^{\varepsilon}(t, x) \partial_{t} \phi(t, x) d t d x & =\frac{1}{h}\left(\int_{\left(0, T_{0}\right) \times \mathbb{R}^{d}} \bar{u}^{\varepsilon}(t, x) \eta_{h}(t, x) d t d x-\int_{\left(0, T_{0}\right) \times \mathbb{R}^{d}} \bar{u}^{\varepsilon}(t, x) \eta_{h}(t-h, x) d t d x\right) \\
& =\frac{1}{h}\left(\int_{\left(0, T_{0}\right) \times \mathbb{R}^{d}} \bar{u}^{\varepsilon}(t, x) \eta_{h}(t, x) d t d x-\int_{\left(0, T_{0}\right) \times \mathbb{R}^{d}} \bar{u}^{\varepsilon}(t+h, x) \eta_{h}(t, x) d t d x\right) \\
& =\int_{\left(0, T_{0}\right) \times \mathbb{R}^{d}} \partial_{t} \bar{u}_{h}^{\varepsilon}(t, x) \eta_{h}(t, x) d t d x,
\end{aligned}
$$

for $\bar{u}_{h}^{\varepsilon}(t, x)=\frac{1}{h} \int_{t}^{t+h} \bar{u}^{\varepsilon}(s, x) d s$. In the same manner, we have

$$
\int_{\left(0, T_{0}\right) \times \mathbb{R}^{d}} \frac{1}{2} \alpha_{\varepsilon}(v(t, x))\left(\nabla_{x} \bar{u}^{\varepsilon}(t, x) \cdot \nabla_{x} \phi(t, x)\right) d t d x=\int_{\left(0, T_{0}\right) \times \mathbb{R}^{d}} \frac{1}{2}\left(\alpha_{\varepsilon}(v) \nabla_{x} \bar{u}^{\varepsilon}\right)_{h}(t, x) \cdot \nabla_{x} \eta_{h}(t, x) d t d x
$$

where

$$
\left(\alpha_{\varepsilon}(v) \nabla_{x} \bar{u}^{\varepsilon}\right)_{h}(t, x):=\frac{1}{h} \int_{t}^{t+h} \alpha_{\varepsilon}(v(s, x)) \nabla_{x} \bar{u}^{\varepsilon}(s, x) d s .
$$

Therefore

$$
0=\int_{\left(0, T_{0}\right) \times \mathbb{R}^{d}} \partial_{t} \bar{u}_{h}^{\varepsilon}(t, x) \eta_{h}(t, x)+\frac{1}{2}\left(\alpha_{\varepsilon}(v) \nabla_{x} \bar{u}^{\varepsilon}\right)_{h}(t, x) \cdot \nabla_{x} \eta_{h}(t, x) d t d x .
$$

Extending the previous equality from $\eta_{h} \in \mathcal{C}_{c}^{\infty}\left(\left(0, T_{0}-h\right) \times \mathbb{R}^{d}\right)$ to $\eta \in L^{2}\left(\left(0, T_{0}\right) ; H^{1}\left(\mathbb{R}^{d}\right)\right)$ by density, it follows that

$$
0=\int_{\left(0, T_{0}\right) \times \mathbb{R}^{d}} \partial_{t} \bar{u}_{h}^{\varepsilon}(t, x) \eta(t, x)+\frac{1}{2}\left(\alpha_{\varepsilon}(v) \nabla_{x} \bar{u}^{\varepsilon}\right)_{h}(t, x) \cdot \nabla_{x} \eta(t, x) d t d x .
$$

Next replacing $\eta=\bar{u}_{h}^{\varepsilon}$, we get

$$
\begin{aligned}
0 & =\int_{\left(0, T_{0}\right) \times \mathbb{R}^{d}} \partial_{t} \bar{u}_{h}^{\varepsilon}(t, x) \bar{u}_{h}^{\varepsilon}(t, x)+\frac{1}{2}\left(\alpha_{\varepsilon}(v) \nabla_{x} \bar{u}^{\varepsilon}\right)_{h}(t, x) \cdot \nabla_{x} \bar{u}_{h}^{\varepsilon}(t, x) d t d x \\
& =\int_{\mathbb{R}^{d}}\left(\bar{u}_{h}^{\varepsilon}\left(T_{0}, x\right)\right)^{2} d x-\int_{\mathbb{R}^{d}}\left(\bar{u}_{h}^{\varepsilon}(0, x)\right)^{2} d x+\int_{\left(0, T_{0}\right) \times \mathbb{R}^{d}} \frac{1}{2}\left(\alpha_{\varepsilon}(v) \nabla_{x} \bar{u}^{\varepsilon}\right)_{h}(t, x) \cdot \nabla_{x} \bar{u}_{h}^{\varepsilon}(t, x) d t d x
\end{aligned}
$$

Since

$$
\begin{aligned}
& \left|\int_{\mathbb{R}^{d}}\left(\bar{u}_{h}^{\varepsilon}\left(T_{0}, x\right)\right)^{2} d x-\int\left(\bar{u}^{\varepsilon}\left(T_{0}, x\right)\right)^{2} d x\right| \\
& =\left|\int_{\mathbb{R}^{d}}\left(\int_{0}^{1}\left(\bar{u}^{\varepsilon}\left(T_{0}+h s, x\right) d s-\bar{u}^{\varepsilon}\left(T_{0}, x\right)\right)\right)\left(\int_{0}^{1}\left(\bar{u}^{\varepsilon}\left(T_{0}+h s, x\right) d s+\bar{u}^{\varepsilon}\left(T_{0}, x\right)\right)\right) d x\right| \\
& \quad \leq \sqrt{\int_{0}^{1}\left\|\bar{u}^{\varepsilon}\left(T_{0}+h s\right)-\bar{u}^{\varepsilon}\left(T_{0}\right)\right\|_{L^{2}\left(\mathbb{R}^{d}\right)}^{2}} d s \sqrt{\int_{0}^{1}\left\|\bar{u}^{\varepsilon}\left(T_{0}+h s\right)+\bar{u}^{\varepsilon}\left(T_{0}\right)\right\|_{L^{2}\left(\mathbb{R}^{d}\right)}^{2} d s,}
\end{aligned}
$$

we have

$$
\lim _{h \rightarrow 0} \int_{\mathbb{R}^{d}}\left(\bar{u}_{h}^{\varepsilon}\left(T_{0}, x\right)\right)^{2} d x=\int_{\mathbb{R}^{d}}\left(\bar{u}^{\varepsilon}\left(T_{0}, x\right)\right)^{2} d x
$$


Similarly,

$$
\lim _{h \rightarrow 0} \int_{\mathbb{R}^{d}}\left(\bar{u}_{h}^{\varepsilon}(0, x)\right)^{2} d x=\int_{\mathbb{R}^{d}}\left(\bar{u}_{0}^{\varepsilon}(x)\right)^{2} d x
$$

and

$$
\lim _{h \rightarrow 0} \int_{\left(0, T_{0}\right) \times \mathbb{R}^{d}}\left(\frac{1}{2} \alpha_{\varepsilon}(v) \nabla_{x} \bar{u}^{\varepsilon}\right)_{h}(t, x) \cdot \nabla_{x} \bar{u}_{h}^{\varepsilon}(t, x) d t d x=\int_{\left(0, T_{0}\right) \times \mathbb{R}^{d}} \frac{1}{2} \alpha_{\varepsilon}(v(t, x))\left|\nabla_{x} \bar{u}_{h}^{\varepsilon}(t, x)\right|^{2} d t d x,
$$

From which we deduce (2.9).

The non-negativeness of $\bar{u}^{\varepsilon}$ and (2.8) follows from comparison principles: since $\bar{u}^{\varepsilon}$ is in $L^{2}\left((0, T) ; H^{1}\left(\mathbb{R}^{d}\right)\right)$, its negative part $\left.\left(\bar{u}^{\varepsilon}\right)^{-}=\max \left(0,-\bar{u}^{\varepsilon}\right)\right)$ is also in $L^{2}\left((0, T) ; H^{1}\left(\mathbb{R}^{d}\right)\right)$ and $\nabla_{x}\left(\bar{u}^{\varepsilon}\right)^{-}=-\nabla_{x} \bar{u}^{\varepsilon} \mathbb{1}_{\left\{\bar{u}^{\varepsilon} \leq 0\right\}}$. Taking $\eta=\left(\bar{u}^{\varepsilon}\right)^{-}$in (2.13) yields

$$
\begin{aligned}
0 & =\int_{\left(0, T_{0}\right) \times \mathbb{R}^{d}} \partial_{t} \bar{u}_{h}^{\varepsilon}(t, x)\left(\bar{u}^{\varepsilon}\right)^{-}(t, x)+\frac{1}{2}\left(\alpha_{\varepsilon}(v) \nabla_{x} \bar{u}^{\varepsilon}\right)_{h}(t, x) \cdot \nabla_{x}\left(\bar{u}^{\varepsilon}\right)^{-}(t, x) d t d x \\
& =-\int_{\mathbb{R}^{d}}\left(\left(\bar{u}_{h}^{\varepsilon}\right)^{-}\left(T_{0}, x\right)\right)^{2} d x+\int_{\mathbb{R}^{d}}\left(\left(\bar{u}_{h}^{\varepsilon}\right)^{-}(0, x)\right)^{2} d x+\int_{\left(0, T_{0}\right) \times \mathbb{R}^{d}} \frac{1}{2}\left(\alpha_{\varepsilon}(v) \nabla_{x} \bar{u}^{\varepsilon}\right)_{h}(t, x) \cdot \nabla_{x}\left(\bar{u}^{\varepsilon}\right)^{-}(t, x) d t d x .
\end{aligned}
$$

Replicating the same arguments as for (2.14),

$$
\begin{aligned}
\lim _{h \rightarrow 0} \int_{\mathbb{R}^{d}}\left(\left(\bar{u}_{h}^{\varepsilon}\right)^{-}\left(T_{0}, x\right)\right)^{2} d x & =\int_{\mathbb{R}^{d}}\left(\left(\bar{u}^{\varepsilon}\right)^{-}\left(T_{0}, x\right)\right)^{2} d x, \\
\lim _{h \rightarrow 0} \int_{\mathbb{R}^{d}}\left(\left(\bar{u}_{h}^{\varepsilon}\right)^{-}(0, x)\right)^{2} d x & =\int_{\mathbb{R}^{d}}\left(\left(u_{0}\right)^{-}(x)\right)^{2} d x, \\
\lim _{h \rightarrow 0} \int_{\left(0, T_{0}\right) \times \mathbb{R}^{d}} \frac{1}{2}\left(\alpha_{\varepsilon}(v) \nabla_{x} \bar{u}^{\varepsilon}\right)_{h}(t, x) \cdot \nabla_{x}\left(\bar{u}_{h}^{\varepsilon}\right)^{-}(t, x) d t d x & =\int_{\left(0, T_{0}\right) \times \mathbb{R}^{d}} \frac{1}{2} \alpha_{\varepsilon}(v(t, x)) \nabla_{x} \bar{u}^{\varepsilon}(t, x) \cdot \nabla_{x}\left(\bar{u}_{h}^{\varepsilon}\right)^{-}(t, x) d t d x \\
& =-\int_{\left(0, T_{0}\right) \times \mathbb{R}^{d}} \frac{1}{2} \alpha_{\varepsilon}(v(t, x))\left|\nabla_{x}\left(\bar{u}_{h}^{\varepsilon}\right)^{-}(t, x)\right|^{2} d t d x .
\end{aligned}
$$

Consequently,

$$
0=-\int_{\mathbb{R}^{d}}\left(\left(\bar{u}^{\varepsilon}\right)^{-}\left(T_{0}, x\right)\right)^{2} d x+\int_{\mathbb{R}^{d}}\left(\left(u_{0}\right)^{-}(x)\right)^{2} d x-\int_{\left(0, T_{0}\right) \times \mathbb{R}^{d}} \frac{1}{2} \alpha_{\varepsilon}(v(t, x))\left|\nabla_{x}\left(\bar{u}_{h}^{\varepsilon}\right)^{-}(t, x)\right|^{2} d t d x .
$$

Since $u_{0} \geq 0$ and $\alpha_{\varepsilon}$ is non-negative, we deduce immediately that $\left(\bar{u}^{\varepsilon}\right)^{-}=0$ on $(0, T) \times \mathbb{R}^{d}$.

For the proof of (2.8), we proceed similarly: we set $K=\left\|u_{0}\right\|_{L^{\infty}}$. Then the positive part of $\bar{u}^{\varepsilon}(t, x)-K$,

$$
\left(\bar{u}^{\varepsilon}(t, x)-K\right)^{+}
$$

is in $L^{2}\left((0, T) ; H^{1}\left(\mathbb{R}^{d}\right)\right)$. This statement simply follows from the observation that

$$
\left|\left(\bar{u}^{\varepsilon}(t, x)-K\right)^{+}\right|^{2} \leq\left|\bar{u}^{\varepsilon}(t, x)\right|^{2}, \text { a.e. on }(0, T) \times \mathbb{R}^{d},
$$

and that

$$
\nabla_{x}\left(\bar{u}^{\varepsilon}-K\right)^{+}=\nabla_{x} \bar{u}^{\varepsilon} \mathbb{1}_{\left\{\bar{u}^{\varepsilon}-K \geq 0\right\}} .
$$

By plugging $\eta=\left(\bar{u}^{\varepsilon}-K\right)^{+}$into (2.13), we get

$$
0=\int_{\left(0, T_{0}\right) \times \mathbb{R}^{d}} \partial_{t} \bar{u}_{h}^{\varepsilon}(t, x)\left(\bar{u}^{\varepsilon}(t, x)-K\right)^{+}(t, x)+\frac{1}{2}\left(\alpha_{\varepsilon}(v) \nabla_{x} \bar{u}^{\varepsilon}\right)_{h}(t, x) \cdot \nabla_{x}\left(\bar{u}^{\varepsilon}(t, x)-K\right)^{+}(t, x) d t d x .
$$

Taking the limit $h \rightarrow 0$ of the above expression yields

$$
\begin{aligned}
0= & \int_{\mathbb{R}^{d}}\left(\left(\bar{u}^{\varepsilon}(t, x)-K\right)^{+}\right)^{2}(t, x) d x-\int_{\mathbb{R}^{d}}\left(\left(u_{0}(x)-K\right)^{+}\right)^{2}(t, x) d x \\
& +\int_{\left(0, T_{0}\right) \times \mathbb{R}^{d}} \frac{1}{2} \alpha_{\varepsilon}(v(t, x))\left|\nabla_{x}\left(\bar{u}^{\varepsilon}(t, x)-K\right)^{+}\right|^{2}(t, x) d t d x .
\end{aligned}
$$

Since $\left(u_{0}(x)-K\right)^{+}=0$ for a.a. $x \in \mathbb{R}^{d},(2.8)$ follows. 


\subsubsection{Proof of Lemma 2.5}

The existence of a weak solution to the non linear PDE (2.6) will be deduced from the following fixed point theorem that we apply to the mapping $A: v \in \mathcal{X} \mapsto A(v) \in \mathcal{X}$, for

$$
\mathcal{X}=\left\{v \in L^{2}\left((0, T) ; H^{1}\left(\mathbb{R}^{d}\right)\right): v \geq 0 \text { a.e. on }(0, T) \times \mathbb{R}^{d} \text { and }\|v\|_{L^{\infty}\left((0, T) \times \mathbb{R}^{d}\right)} \leq\left\|u_{0}\right\|_{L^{\infty}\left(\mathbb{R}^{d}\right)}\right\}
$$

equipped with the \|\|$_{L^{2}\left((0, T) ; H^{1}\left(\mathbb{R}^{d}\right)\right)}$-norm, and where $A$ assigns to any nonnegative $v \in \mathcal{X}$, the weak solution $A(v)$ to the linear PDE (2.7) given by Lemma 2.4 with the estimates (2.8) and (2.9).

Theorem 2.7 (Schaefer's fixed point Theorem, see [11], Theorem 4, Chapter 9, Section 2). Let $\mathcal{X}$ be a Banach space and $A: \mathcal{X} \rightarrow \mathcal{X}$ be a continuous and compact mapping such that the set

$$
\mathcal{I}=\{u \in \mathcal{X} \text { s.t. there exists } 0 \leq \lambda \leq 1 \text { with } u=\lambda A(u)\}
$$

is bounded in $\mathcal{X}$. Then A has a fixed point in $\mathcal{X}$.

The continuity of $A$. Let $\left\{v_{n}\right\}_{n}$ converge to $v_{\infty}$ in $L^{2}\left((0, T) ; H^{1}\left(\mathbb{R}^{d}\right)\right)$. Then, since $A\left(v_{n}\right)$ and $A\left(v_{\infty}\right)$ are weak solution to (2.7) endowed with the diffusion coefficient $\sigma_{\varepsilon}\left(v_{n}\right)$ and $\sigma_{\varepsilon}\left(v_{\infty}\right)$ respectively, we have

$$
0=\int_{(0, T) \times \mathbb{R}^{d}}\left(A\left(v_{n}\right)-A\left(v_{\infty}\right)\right) \partial_{t} f-\left(\frac{1}{2} \alpha_{\varepsilon}\left(v_{n}\right) \nabla_{x} A\left(v_{n}\right)-\frac{1}{2} \alpha_{\varepsilon}\left(v_{\infty}\right) \nabla_{x} A\left(v_{\infty}\right)\right) \nabla_{x} f
$$

for all $f \in \mathcal{C}_{c}^{\infty}\left((0, T) \times \mathbb{R}^{d}\right)$. Replicating the arguments for the energy estimate (2.9) in Lemma 2.4, we deduce that

$$
\begin{aligned}
& \int_{\mathbb{R}^{d}}\left|A\left(v_{n}\right)\left(T_{0}\right)-A\left(v_{\infty}\right)\left(T_{0}\right)\right|^{2}+\int_{\left(0, T_{0}\right) \times \mathbb{R}^{d}} \frac{1}{2} \alpha_{\varepsilon}\left(v_{n}\right)\left|\nabla_{x} A\left(v_{n}\right)-\nabla_{x} A\left(v_{\infty}\right)\right|^{2} \\
& =\int_{\left(0, T_{0}\right) \times \mathbb{R}^{d}} \frac{1}{2}\left(\alpha_{\varepsilon}\left(v_{n}\right)-\alpha_{\varepsilon}\left(v_{\infty}\right)\right)\left(\nabla_{x} A\left(v_{n}\right)-\nabla_{x} A\left(v_{\infty}\right)\right) \cdot \nabla_{x} A\left(v_{n}\right) \\
& \quad \leq \frac{1}{\varepsilon} \int_{\left(0, T_{0}\right) \times \mathbb{R}^{d}} \frac{1}{2}\left|\alpha_{\varepsilon}\left(v_{n}\right)-\alpha_{\varepsilon}\left(v_{\infty}\right)\right|^{2}\left|\nabla_{x} A\left(v_{n}\right)\right|^{2}+\frac{\varepsilon}{4} \int_{\left(0, T_{0}\right) \times \mathbb{R}^{d}}\left|\nabla_{x} A\left(v_{n}\right)-\nabla_{x} A\left(v_{\infty}\right)\right|^{2} .
\end{aligned}
$$

Since $v_{n} \rightarrow v_{\infty}$ in $L^{2}\left((0, T) \times \mathbb{R}^{d}\right)$, there exists a subsequence $\left\{v_{n_{k}}\right\}_{k}$ such that $v_{n_{k}} \rightarrow v_{\infty}$ a.e. on $(0, T) \times \mathbb{R}^{d}$ and such that $\sup _{k}\left|v_{n_{k}}\right|$ is in $L^{2}\left((0, T) \times \mathbb{R}^{d}\right)$. Replacing $v_{n}$ by $v_{n_{k}}$ in the preceding inequality and applying the Lebesgue's dominated convergence theorem, we deduce that $\lim _{k} A\left(v_{n_{k}}\right)=A\left(v_{\infty}\right)$ in $L^{2}\left((0, T) ; H^{1}\left(\mathbb{R}^{d}\right)\right)$. With the same reasoning, for any subsequence of $\left\{A\left(v_{n}\right)\right\}_{n}$, we can extract a subsequence which converges to $A\left(v_{\infty}\right)$. Since $\mathcal{X}$ is a closed subset of $L^{2}\left((0, T) ; H^{1}\left(\mathbb{R}^{d}\right)\right)$, this implies the continuity of $A$.

The compactness of $A$. Owing to Lemma 2.4, for any converging sequence $\left\{v_{n}\right\}_{n}$ in $\mathcal{X},\left\{A\left(v_{n}\right)\right\}_{n}$ is a bounded sequence in $L^{2}\left((0, T) ; H^{1}\left(\mathbb{R}^{d}\right)\right)$. We can then extract a subsequence $\left\{A\left(v_{n_{k}}\right)\right\}_{k}$ converging to some limit $A_{\infty}$ in the weak topology of $L^{2}\left((0, T) ; H^{1}\left(\mathbb{R}^{d}\right)\right)$. In particular, since $\sup _{k}\left\|A\left(v_{n_{k}}\right)\right\|_{L^{2}\left((0, T) \times \mathbb{R}^{d}\right)}$ is finite, $\| \nabla_{x} A\left(v_{n_{k}}\right)-$ $A_{\infty} \|_{L^{2}\left((0, T) \times \mathbb{R}^{d}\right)}$ tends to 0 as $k$ tends to $\infty$. The uniform bound (2.8) naturally holds for $v_{\infty}$.

The boundedness of $\mathcal{I}$. Finally, let us consider the set

$$
\mathcal{I}:=\{u \in \mathcal{X} \text { s.t. there exists } 0 \leq \lambda \leq 1 \text { with } u=\lambda A(u)\} .
$$

Excluding the trivial case $\lambda=0$, one can check that for all $u_{\lambda} \in \mathcal{X}$ such that $u_{\lambda}=\lambda A\left(u_{\lambda}\right), u_{\lambda}$ is the $L^{2}\left((0, T) ; H^{1}\left(\mathbb{R}^{d}\right)\right)$ weak solution to

$$
\left\{\begin{array}{l}
\frac{1}{\lambda} \frac{\partial u_{\lambda}}{\partial t}-\frac{1}{2} \triangle\left(\sigma_{\varepsilon}^{2}\left(u_{\lambda}\right) \frac{u_{\lambda}}{\lambda}\right)=0, \text { on }(0, T) \times \mathbb{R}^{d}, \\
\frac{1}{\lambda} u_{\lambda}(0, x)=u_{0}(x), x \in \mathbb{R}^{d}
\end{array}\right.
$$


given as in Lemma 2.4. Equivalently,

$$
\begin{aligned}
\int_{\mathbb{R}^{d}} & u_{\lambda}\left(T_{0}, x\right) f\left(T_{0}, x\right) d x-\lambda \int_{\mathbb{R}^{d}} u_{0}(x) f(0, x) d x \\
& =\int_{\left(0, T_{0}\right) \times \mathbb{R}^{d}} u_{\lambda}(t, x) \partial_{t} f(t, x)-\frac{1}{2} \alpha_{\varepsilon}\left(u_{\lambda}\right) \nabla_{x} u_{\lambda}(t, x) \cdot \nabla_{x} f(t, x) d t d x .
\end{aligned}
$$

The energy estimate (2.9) from Lemma 2.4 then ensures that $\left\|u_{\lambda}\right\|_{L^{2}\left((0, T) \times \mathbb{R}^{d}\right)}^{2} \leq \lambda^{2} T\left\|u_{0}\right\|_{L^{2}\left(\mathbb{R}^{d}\right)}^{2}$ from which we conclude on the boundedness of $\mathcal{I}$.

The Schaefer Theorem ensures the existence of a $L^{2}\left((0, T) ; H^{1}\left(\mathbb{R}^{d}\right)\right)$-weak solution to (2.6), for which the $L^{2}$ (and $L^{\infty}$ ) estimate established in Lemma 2.4 still hold true.

For the estimate (2.12), we replicate the proof arguments of Vasquez [34] (see Chapter 5). Owing to assumptions $\left(A_{0}\right)$ and $\left(A_{1}\right)$,

$$
\int_{\mathbb{R}^{d}} \Psi_{\varepsilon}\left(u_{0}(x)\right) d x=\int_{\mathbb{R}^{d}} \int_{0}^{u_{0}(x)} \Phi_{\varepsilon}(r) d r d x \leq \frac{1}{2}\left(\sup _{0 \leq r \leq\left\|u_{0}\right\|_{L^{\infty}\left(\mathbb{R}^{d}\right)}}(\sigma(r))^{2}+\varepsilon\right)\left\|u_{0}\right\|_{L^{2}\left(\mathbb{R}^{d}\right)}^{2} .
$$

Replicating the proof arguments of (2.13), for all $\eta \in \mathcal{C}_{c}^{\infty}\left((0, T) \times \mathbb{R}^{d}\right)$, for $h>0, T_{0}>0$ such that $T_{0}+h \leq T$,

$$
0=\int_{\left(0, T_{0}\right) \times \mathbb{R}^{d}} \partial_{t} u_{h}^{\varepsilon}(t, x) \eta(t, x)+\frac{1}{2}\left(\alpha_{\varepsilon}\left(u^{\varepsilon}\right) \nabla_{x} u^{\varepsilon}\right)_{h}(t, x) \cdot \nabla_{x} \eta(t, x) d t d x,
$$

with $u_{h}^{\varepsilon}(t, x):=\frac{1}{h} \int_{t}^{t+h} u^{\varepsilon}(s, x) d s$, and

$$
\left(\alpha_{\varepsilon}\left(u^{\varepsilon}\right) \nabla_{x} u^{\varepsilon}\right)_{h}(t, x):=\frac{1}{h} \int_{t}^{t+h} \alpha_{\varepsilon}\left(u^{\varepsilon}(s, x)\right) \nabla_{x} u^{\varepsilon}(s, x) d s .
$$

Observing that

$$
\left\|\Phi_{\varepsilon}\left(u_{h}^{\varepsilon}\right)\right\|_{L^{2}\left((0, T) \times \mathbb{R}^{d}\right)}=\left\|\left(\sigma^{2}\left(u^{\varepsilon}\right)+\varepsilon\right) u_{h}^{\varepsilon}\right\|_{L^{2}\left((0, T) \times \mathbb{R}^{d}\right)} \leq \frac{1}{2}\left(\sup _{0 \leq r \leq\left\|u_{0}\right\|_{L^{\infty}\left(\mathbb{R}^{d}\right)}}(\sigma(r))^{2}+\varepsilon\right)\left\|u^{\varepsilon}\right\|_{L^{2}\left((0, T) \times \mathbb{R}^{d}\right)},
$$

and that

$$
\begin{aligned}
\left\|\nabla_{x} \Phi_{\varepsilon}\left(u_{h}^{\varepsilon}\right)\right\|_{L^{2}\left((0, T) \times \mathbb{R}^{d}\right)}^{2} & =\left\|\Phi_{\varepsilon}^{\prime}\left(u_{h}^{\varepsilon}\right) \nabla_{x} u_{h}^{\varepsilon}\right\|_{L^{2}\left((0, T) \times \mathbb{R}^{d}\right)}^{2}=\left\|\alpha_{\varepsilon}\left(u^{\varepsilon}\right) \nabla_{x} u_{h}^{\varepsilon}\right\|_{L^{2}\left((0, T) \times \mathbb{R}^{d}\right)}^{2} \\
& \leq\left(\left\|\alpha\left(u^{\epsilon}\right)\right\|_{L^{\infty}\left(\mathbb{R}^{d}\right)}^{2}+\varepsilon^{2}\right)\left\|\nabla_{x} u^{\varepsilon}\right\|_{L^{2}\left((0, T) \times \mathbb{R}^{d}\right)}^{2},
\end{aligned}
$$

$\Phi_{\varepsilon}\left(u_{h}^{\varepsilon}\right)$ is in $L^{2}\left((0, T) ; H^{1}\left(\mathbb{R}^{d}\right)\right)$. Taking $\eta=\Phi_{\varepsilon}\left(u_{h}^{\varepsilon}\right)$ in $(2.15)$

$$
\begin{aligned}
0 & =\int_{\left(0, T_{0}\right) \times \mathbb{R}^{d}} \partial_{t} u_{h}^{\varepsilon}(t, x) \Phi_{\varepsilon}\left(u_{h}^{\varepsilon}\right)(t, x)+\frac{1}{2}\left(\alpha_{\varepsilon}\left(u^{\varepsilon}\right) \nabla_{x} u^{\varepsilon}\right)_{h}(t, x) \cdot \nabla_{x} \Phi_{\varepsilon}\left(u_{h}^{\varepsilon}\right)(t, x) d t d x \\
& =\int_{\left(0, T_{0}\right) \times \mathbb{R}^{d}} \partial_{t} \Psi_{\varepsilon}\left(u_{h}^{\varepsilon}\right)(t, x)+\frac{1}{2} \int_{\left(0, T_{0}\right) \times \mathbb{R}^{d}}\left(\nabla_{x} \Phi_{\varepsilon}\left(u_{h}^{\varepsilon}\right)\right)_{h}(t, x) \cdot \nabla_{x} \Phi_{\varepsilon}\left(u_{h}^{\varepsilon}\right)(t, x) d t d x \\
& =\left\|\Psi_{\varepsilon}\left(u_{h}^{\varepsilon}\right)\left(T_{0}\right)\right\|_{L^{1}\left(\mathbb{R}^{d}\right)}-\left\|\Psi_{\varepsilon}\left(u_{h}^{\varepsilon}\right)(0)\right\|_{L^{1}\left(\mathbb{R}^{d}\right)}+\frac{1}{2} \int_{\left(0, T_{0}\right) \times \mathbb{R}^{d}}\left(\nabla_{x} \Phi_{\varepsilon}\left(u_{h}^{\varepsilon}\right)\right)_{h}(t, x) \cdot \nabla_{x} \Phi_{\varepsilon}\left(u_{h}^{\varepsilon}\right)(t, x) d t d x .
\end{aligned}
$$

Taking the limit $h \rightarrow 0$ it follows that

$$
0=\left\|\Psi_{\varepsilon}\left(u^{\varepsilon}\right)\left(T_{0}\right)\right\|_{L^{1}\left(\mathbb{R}^{d}\right)}-\left\|\Psi_{\varepsilon}\left(u_{0}\right)\right\|_{L^{1}\left(\mathbb{R}^{d}\right)}+\frac{1}{2} \int_{\left(0, T_{0}\right) \times \mathbb{R}^{d}}\left|\nabla_{x} \Phi_{\varepsilon}\left(u_{h}^{\varepsilon}\right)\right|^{2}(t, x) d t d x .
$$

Finally, since $u^{\epsilon}$ is a $L^{2}\left((0, T) ; H^{1}\left(\mathbb{R}^{d}\right)\right)$-solution to (2.6), the property $u^{\epsilon} \in \mathcal{C}\left([0, T] ; L^{2}\left(\mathbb{R}^{d}\right)\right)$ can be again obtained by following [23], Theorem 2.1, Chapter 4 . This ends the proof. 


\subsection{Existence result for (2.1)}

The existence result in Theorem 2.3 will be deduced from the asymptotic behavior (up to a subsequence extraction) of the solution to (2.5) as $\varepsilon \rightarrow 0$. The precise result is the following:

Proposition 2.8. Assume $\left(A_{0}\right),\left(A_{1}\right)$ and $\left(A_{2}\right)$. Consider $\left(X_{t}^{\varepsilon} ; t \geq 0\right)$ solution to (2.5) given by Proposition 2.6. The sequence $\left\{\left(P^{\varepsilon}, u^{\varepsilon}\right)\right\}_{\varepsilon>0}$, defined by

$$
P^{\varepsilon}=\operatorname{Law}\left(X_{t}^{\varepsilon} ; 0 \leq t \leq T\right),
$$

and $u^{\varepsilon}$ given as in Lemma 2.5 , admits a weakly converging subsequence $\left\{\left(P^{\varepsilon_{k}}, u^{\varepsilon_{k}}\right)\right\}_{k}$ such that $u^{0}=\lim _{k} u^{\varepsilon_{k}}$ in $L^{2}\left((0, T) \times \mathbb{R}^{d}\right)$, is a $L^{2}\left((0, T) \times \mathbb{R}^{d}\right)$-weak solution to

$$
\left\{\begin{array}{l}
\frac{\partial u}{\partial t}-\frac{1}{2} \triangle_{x}\left(\sigma^{2}(u) u\right)=0, \text { on }(0, T) \times \mathbb{R}^{d} \\
u(0, x)=u_{0}(x), x \in \mathbb{R}^{d}
\end{array}\right.
$$

and $P^{0}=\lim _{k} P^{\varepsilon_{k}}$ is solution to the following martingale problem $(M P)$ : let $(x(t) ; 0 \leq t \leq T)$ denotes the canonical process on $\mathcal{C}\left([0, T] ; \mathbb{R}^{d}\right)$, we have

(MP)-(i) For all $0 \leq t \leq T, P^{0}(x(t) \in d x)=u^{0}(t, x) d x$;

(MP)-(ii) For all $f \in \mathcal{C}_{c}^{2}\left(\mathbb{R}^{d}\right)$,

$$
t \mapsto f(x(t))-f(x(0))-\frac{1}{2} \int_{0}^{t} \sigma^{2}\left(u^{0}(s, x)\right) \triangle_{x} f(x(s)) d s
$$

is a continuous martingale under $P^{0}$.

From Lemma 2.5, the $L^{2}\left((0, T) \times \mathbb{R}^{d}\right)$-convergence of $u^{\varepsilon}$ to $u^{0}$ ensures that

$$
\left\|u^{0}\right\|_{L^{\infty}\left((0, T) \times \mathbb{R}^{d}\right)}<\infty, \text { and } \int_{\mathbb{R}^{d}}\left|\nabla \Phi_{\varepsilon}\left(u^{0}(t, x)\right)\right|^{2} d t d x<\infty .
$$

In particular under $\left(A_{2}\right)$, the control of $\left\|\nabla \Phi_{\varepsilon}\left(u^{0}(t, x)\right)\right\|_{L^{2}\left((0, T) \times \mathbb{R}^{d}\right)}$ yields to the estimate:

$$
\eta\left\|\nabla_{x} u^{0}\right\|_{L^{2}\left((0, T) \times \mathbb{R}^{d}\right)} \leq\left\|\alpha_{\varepsilon}\left(u^{0}\right) \nabla_{x} u^{0}\right\|_{L^{2}\left((0, T) \times \mathbb{R}^{d}\right)} \leq\left\|\nabla_{x} \Phi_{\varepsilon}\left(u^{0}\right)\right\|_{L^{2}\left((0, T) \times \mathbb{R}^{d}\right)}<\infty .
$$

Therefore

Corollary 2.9. The time marginal densities $u^{0}(t)$ of $\left(X_{t}^{0} ; 0 \leq t \leq T\right)$ given in Proposition 2.8 are in $L^{\infty}((0, T) \times$ $\left.\mathbb{R}^{d}\right) \cap L^{2}\left((0, T) ; H^{1}\left(\mathbb{R}^{d}\right)\right)$.

Proof of Proposition 2.8. Owing to (2.11), we deduce that $\left\{u^{\varepsilon}\right\}_{\varepsilon}$ is relatively compact for the weak topology in $L^{2}\left((0, T) \times \mathbb{R}^{d}\right)$. Denote by $\left\{u^{\varepsilon_{k}}\right\}_{k}$ a (weakly) converging subsequence and $u^{0}$ its limit. Under the assumption $\left(A_{2}\right)$, the estimate (2.12) ensures that

$$
\sup _{\varepsilon}\left\|\nabla_{x} u^{\varepsilon}\right\|_{L^{2}\left((0, T) \times \mathbb{R}^{d}\right)} \leq \frac{1}{\eta} \sup _{\varepsilon}\left\|\alpha\left(u^{\varepsilon}\right) \nabla_{x} u^{\varepsilon}\right\|_{L^{2}\left((0, T) \times \mathbb{R}^{d}\right)}=\frac{1}{\eta} \sup _{\varepsilon}\left\|\nabla_{x} \Phi\left(u^{\varepsilon}\right)\right\|_{L^{2}\left((0, T) \times \mathbb{R}^{d}\right)}<\infty,
$$

so that $\lim _{k}\left\|u^{\varepsilon_{k}}-u^{0}\right\|_{L^{2}\left((0, T) \times \mathbb{R}^{d}\right)}=0$. We can further extract a converging subsequence such that the convergence holds a.e. on $(0, T) \times \mathbb{R}^{d}$ and $\sup _{k}\left|u^{\varepsilon_{k}}\right| \in L^{2}\left((0, T) \times \mathbb{R}^{d}\right)$. Since $\sigma_{\varepsilon}\left(u^{\varepsilon}\right)$ is bounded and by $\left(A_{0}\right)$, according to the Kolmogorov-Centov criterion, the sequence $\left\{P^{\varepsilon}\right\}_{\varepsilon}$ is tight on $\left(\mathcal{C}\left([0, T] ; \mathbb{R}^{d}\right), \mathcal{B}\left(\mathcal{C}\left([0, T] ; \mathbb{R}^{d}\right)\right)\right)$. Denote for simplicity by $\left\{\left(P^{\varepsilon_{k}}, u^{\varepsilon_{k}}\right)\right\}_{k}$ a converging pair of $\left\{\left(P^{\varepsilon}, u^{\varepsilon}\right)\right\}_{\varepsilon}$ and by $\left(P^{0}, u^{0}\right)$ its limit. Since $P^{\varepsilon_{k}}(x(t) \in d x)=u^{\varepsilon_{k}}(t, x) d x$ the $L^{2}$-convergence of $u^{\varepsilon_{k}}$ to $u^{0}$ and the convergence of the time marginal distributions of $P^{\varepsilon_{k}}$ to $P^{0}$ ensure that $P^{0}(x(t) \in d x)=u^{0}(t, x) d x$ for a.e. $0 \leq t \leq T$. 
Coming back to (2.6) and taking the limit $k \rightarrow 0$ in the expression,

$$
0=\int_{(0, T) \times \mathbb{R}^{d}} u^{\varepsilon}(t, x) \partial_{t} f(t, x)-\frac{1}{2} \alpha_{\varepsilon}\left(u^{\varepsilon}(t, x)\right) \nabla_{x} u^{\varepsilon}(t, x) \cdot \nabla_{x} f(t, x) d t d x,
$$

for $f \in \mathcal{C}_{c}^{\infty}\left((0, T) \times \mathbb{R}^{d}\right)$, and owing to the continuity of $\sigma$, we deduce that $u^{0}$ is a weak $L^{2}\left((0, T) \times \mathbb{R}^{d}\right)$-solution to (2.16).

In order to identify $P^{0}$ as the solution of the martingale problem $(M P)$, it is sufficient to show that, for all $0 \leq s<$ $t \leq T, \psi: \mathcal{C}\left([0, s] ; \mathbb{R}^{d}\right) \rightarrow \mathbb{R}$ bounded and continuous, $f \in \mathcal{C}_{c}^{2}\left(\mathbb{R}^{d}\right)$,

$$
\begin{aligned}
& \lim _{k} \mathbb{E}_{P^{\varepsilon_{k}}}\left[\psi(x(r) ; 0 \leq r \leq s) \int_{s}^{t} \sigma_{\varepsilon}^{2}\left(u^{\varepsilon}(\theta, x(\theta)) \triangle_{x} f(x(\theta)) d \theta\right]\right. \\
& =\mathbb{E}_{P^{0}}\left[\psi(x(r) ; 0 \leq r \leq s) \int_{s}^{t} \sigma^{2}\left(u^{0}(\theta, x(\theta)) \triangle_{x} f(x(\theta)) d \theta\right] .\right.
\end{aligned}
$$

To this end, let us introduce a smooth approximation of $\sigma_{\varepsilon}^{2}\left(u^{\varepsilon_{k}}\right)$ and $\sigma^{2}\left(u^{0}\right)$ with

$$
\left(\sigma_{\varepsilon}^{2}\left(u^{\varepsilon_{k}}(t)\right)\right)_{\beta}(x):=\left(\phi_{\beta} * \sigma_{\varepsilon}^{2}\left(u^{\varepsilon_{k}}(t)\right)\right)(x), \text { and }\left(\sigma^{2}\left(u^{0}(t)\right)\right)_{\beta}(x):=\left(\phi_{\beta} * \sigma^{2}\left(u^{0}(t)\right)\right)(x),
$$

for $*$ denoting the convolution product on the variable $x \in \mathbb{R}^{d}$ and $\left\{\phi_{\beta}\right\}_{\beta>0}$ a sequence of mollifiers on $\mathbb{R}^{d}$ given by $\phi_{\beta}(y)=\frac{1}{\beta^{d}} \phi\left(\frac{y}{\beta}\right)$ with $\phi \geq 0, \phi \in \mathcal{C}_{c}^{\infty}\left(\mathbb{R}^{d}\right)$ and $\int \phi(y) d y=1$.

Then, we can consider

$$
\begin{aligned}
& \mid \mathbb{E}_{P^{\varepsilon_{k}}}\left[\psi(x(r) ; 0 \leq r \leq s) \int_{s}^{t} \sigma_{\varepsilon}^{2}\left(u^{\varepsilon}(\theta, x(\theta))\right) \triangle_{x} f(x(\theta)) d \theta\right] \\
& \quad-\mathbb{E}_{P^{0}}\left[\psi(x(r) ; 0 \leq r \leq s) \int_{s}^{t} \sigma^{2}\left(u^{0}(\theta, x(\theta))\right) \triangle_{x} f(x(\theta)) d \theta\right] \mid \\
& \leq\left|\mathbb{E}_{P^{\varepsilon_{k}}}\left[\psi(x(r) ; 0 \leq r \leq s) \int_{s}^{t}\left(\sigma_{\varepsilon}^{2}\left(u^{\varepsilon}(\theta, x(\theta))\right)-\left(\sigma_{\varepsilon}^{2}\left(u^{\varepsilon_{k}}(\theta)\right)\right)_{\beta}(x(\theta))\right) \triangle_{x} f(x(\theta)) d \theta\right]\right| \\
& +\mid \mathbb{E}_{P^{\varepsilon_{k}}}\left[\psi(x(r) ; 0 \leq r \leq s) \int_{s}^{t}\left(\sigma_{\varepsilon}^{2}\left(u^{\varepsilon_{k}}(\theta)\right)\right)_{\beta}(x(\theta)) \triangle_{x} f(x(\theta)) d \theta\right] \\
& \quad-\mathbb{E}_{P^{0}}\left[\psi(x(r) ; 0 \leq r \leq s) \int_{s}^{t}\left(\sigma^{2}\left(u^{0}(\theta)\right)\right)_{\beta}(x(\theta)) \triangle_{x} f(x(\theta)) d \theta\right] \mid \\
& +\left|\mathbb{E}_{P^{0}}\left[\psi(x(r) ; 0 \leq r \leq s) \int_{s}^{t}\left(\left(\sigma^{2}\left(u^{0}(\theta)\right)\right)_{\beta}(x(\theta))-\sigma^{2}\left(u^{0}(\theta, x(\theta))\right) \triangle_{x} f(x(\theta))\right) d \theta\right]\right| \\
& =: I_{1}^{\varepsilon, \beta}+I_{2}^{\varepsilon, \beta}+I_{3}^{\beta} .
\end{aligned}
$$

By the weak convergence of $P^{\varepsilon_{k}}$ and since $\left(\sigma_{\varepsilon}^{2}\left(u^{\varepsilon_{k}}(t)\right)\right)_{\beta}(x)$ converges locally to $\left(\sigma^{2}\left(u^{0}(t)\right)\right)_{\beta}(x)$, it follows that $\lim _{\beta \rightarrow 0} \lim _{\varepsilon \rightarrow 0} I_{2}^{\varepsilon, \beta}=0$.

For $I_{1}^{\varepsilon, \beta}$, observe that

$$
\begin{aligned}
I_{1}^{\varepsilon, \beta} & \leq\|\psi\|_{\infty} \mathbb{E}_{P^{\varepsilon_{k}}}\left[\int_{0}^{T}\left|\sigma_{\varepsilon}^{2}\left(u^{\varepsilon}(\theta, x(\theta))\right)-\left(\sigma_{\varepsilon}^{2}\left(u^{\varepsilon_{k}}(\theta)\right)\right)_{\beta}(x(\theta))\right|\left|\triangle_{x} f(x(\theta))\right| d \theta\right] \\
& \leq\|\psi\|_{\infty} \sup _{k}\left\|u^{\varepsilon_{k}}\right\|_{L^{2}\left((0, T) \times \mathbb{R}^{d}\right)} \sqrt{\int_{(0, T) \times \mathbb{R}^{d}}\left|\sigma_{\varepsilon}^{2}\left(u^{\varepsilon}(\theta, x)\right)-\left(\sigma_{\varepsilon}^{2}\left(u^{\varepsilon_{k}}(\theta)\right)\right)_{\beta}(x)\right|^{2}\left|\triangle_{x} f(x)\right|^{2} d x d \theta .}
\end{aligned}
$$

Since

$$
\left|\sigma_{\varepsilon_{k}}^{2}\left(u^{\varepsilon_{k}}(\theta, x)\right)-\left(\sigma_{\varepsilon_{k}}^{2}\left(u^{\varepsilon_{k}}(\theta)\right)\right)_{\beta}(x)\right| \leq \int_{\mathbb{R}^{d}} \phi(y)\left|\sigma_{\varepsilon_{k}}^{2}\left(u^{\varepsilon_{k}}(\theta, x)\right)-\sigma_{\varepsilon_{k}}^{2}\left(u^{\varepsilon_{k}}(\theta, x-\beta y)\right)\right| d y,
$$


we have

$$
\begin{aligned}
& \int_{(0, T) \times \mathbb{R}^{d}}\left|\sigma_{\varepsilon}^{2}\left(u^{\varepsilon}(\theta, x)\right)-\left(\sigma_{\varepsilon}^{2}\left(u^{\varepsilon_{k}}(\theta)\right)\right)_{\beta}(x)\right|^{2}\left|\triangle_{x} f(x)\right|^{2} d x d \theta \\
& \leq \int_{\mathbb{R}^{d}} \phi(y)\left(\int_{(0, T) \times \mathbb{R}^{d}}\left|\sigma_{\varepsilon_{k}}^{2}\left(u^{\varepsilon_{k}}(\theta, x)\right)-\sigma_{\varepsilon_{k}}^{2}\left(u^{\varepsilon_{k}}(\theta, x-\beta y)\right)\right|^{2}\left|\triangle_{x} f(x)\right|^{2} d \theta d x\right) d y .
\end{aligned}
$$

Then we observe that, for all $y \in \mathbb{R}^{d}$,

$$
\begin{aligned}
& \int_{(0, T) \times \mathbb{R}^{d}}\left|\sigma_{\varepsilon_{k}}^{2}\left(u^{\varepsilon_{k}}(\theta, x)\right)-\sigma_{\varepsilon_{k}}^{2}\left(u^{\varepsilon_{k}}(\theta, x-\beta y)\right)\right|^{2}\left|\triangle_{x} f(x)\right|^{2} d \theta d x \\
& \leq \int_{(0, T) \times \mathbb{R}^{d}}\left|\sigma_{\varepsilon_{k}}^{2}\left(u^{\varepsilon_{k}}(\theta, x)\right)-\sigma_{\varepsilon_{k}}^{2}\left(u^{0}(\theta, x)\right)\right|^{2}\left|\triangle_{x} f(x)\right|^{2} d \theta d x \\
& \quad+\int_{(0, T) \times \mathbb{R}^{d}}\left|\sigma_{\varepsilon_{k}}^{2}\left(u^{0}(\theta, x)\right)-\sigma_{\varepsilon_{k}}^{2}\left(u^{0}(\theta, x-\beta y)\right)\right|^{2}\left|\triangle_{x} f(x)\right|^{2} d \theta d x \\
& \quad+\int_{(0, T) \times \mathbb{R}^{d}}\left|\sigma_{\varepsilon_{k}}^{2}\left(u^{\varepsilon_{k}}(\theta, x-\beta y)\right)-\sigma_{\varepsilon_{k}}^{2}\left(u^{\varepsilon_{k}}(\theta, x-\beta y)\right)\right|^{2}\left|\triangle_{x} f(x)\right|^{2} d \theta d x .
\end{aligned}
$$

By continuity of $\sigma$, as $k$ tends to $\infty, \sigma\left(u^{\varepsilon_{k}}\right)$ tends to $\sigma\left(u^{0}\right)$ a.e. on $(0, T) \times \mathbb{R}^{d}$. Therefore, by Lebesgue's dominated convergence theorem, the first expression in the right hand side of (2.18) tends to 0 . In the same way

$$
\begin{aligned}
& \lim _{k} \int_{(0, T) \times \mathbb{R}^{d}}\left|\sigma_{\varepsilon_{k}}^{2}\left(u^{\varepsilon_{k}}(\theta, x-\beta y)\right)-\sigma_{\varepsilon_{k}}^{2}\left(u^{\varepsilon_{k}}(\theta, x-\beta y)\right)\right|^{2}\left|\triangle_{x} f(x)\right|^{2} d \theta d x \\
& =\lim _{k} \int_{(0, T) \times \mathbb{R}^{d}}\left|\sigma_{\varepsilon_{k}}^{2}\left(u^{\varepsilon_{k}}(\theta, x)\right)-\sigma_{\varepsilon_{k}}^{2}\left(u^{\varepsilon_{k}}(\theta, x)\right)\right|^{2}\left|\triangle_{x} f(x+\beta y)\right|^{2} d \theta d x=0 .
\end{aligned}
$$

For the remaining component in (2.18), assuming that the support of $f$ is included in the open ball $B(0, R)$ for some radius $R<\infty$,

$$
\begin{aligned}
& \int_{(0, T) \times \mathbb{R}^{d}}\left|\sigma_{\varepsilon_{k}}^{2}\left(u^{0}(\theta, x)\right)-\sigma_{\varepsilon_{k}}^{2}\left(u^{0}(\theta, x-\beta y)\right)\right|^{2}\left|\triangle_{x} f(x)\right|^{2} d \theta d x \\
& \leq\left\|\triangle_{x} f\right\|_{\infty} \int_{(0, T) \times B(0, R)}\left|\sigma_{\varepsilon_{k}}^{2}\left(u^{0}(\theta, x)\right)-\sigma_{\varepsilon_{k}}^{2}\left(u^{0}(\theta, x-\beta y)\right)\right|^{2} d \theta d x
\end{aligned}
$$

Since $\sigma_{\varepsilon_{k}}^{2}\left(u^{0}\right)$ is bounded, the continuity of

$$
z \in \mathbb{R}^{d} \mapsto \int_{(0, T) \times B(0, R)}\left|\sigma_{\varepsilon_{k}}^{2}\left(u^{0}(\theta, x)\right)-\sigma_{\varepsilon_{k}}^{2}\left(u^{0}(\theta, x-z)\right)\right|^{2} d \theta d x
$$

ensures that

$$
\lim _{\beta \rightarrow 0} \int_{(0, T) \times \mathbb{R}^{d}}\left|\sigma_{\varepsilon_{k}}^{2}\left(u^{0}(\theta, x)\right)-\sigma_{\varepsilon_{k}}^{2}\left(u^{0}(\theta, x-\beta y)\right)\right|^{2}\left|\triangle_{x} f(x)\right|^{2} d \theta d x=0 .
$$

Coming back to (2.18), we deduce that

$$
\lim _{\beta \rightarrow 0} \lim _{\varepsilon \rightarrow 0} \int_{(0, T) \times \mathbb{R}^{d}}\left|\sigma_{\varepsilon_{k}}^{2}\left(u^{\varepsilon_{k}}(\theta, x)\right)-\sigma_{\varepsilon_{k}}^{2}\left(u^{\varepsilon_{k}}(\theta, x-\beta y)\right)\right|^{2}\left|\triangle_{x} f(x)\right|^{2} d \theta d x=0,
$$

and by extension that $\lim _{\beta \rightarrow 0} \lim _{\varepsilon \rightarrow 0} I_{1}^{\varepsilon, \beta}=0$. 
Finally, for $I_{3}^{\beta}$, replicating the arguments for $I_{1}^{\varepsilon_{k}, \beta}$, we have

$$
\begin{aligned}
& \left|\mathbb{E}_{P^{0}}\left[\psi(x(r) ; 0 \leq r \leq s) \int_{s}^{t}\left(\left(\sigma^{2}\left(u^{0}(\theta)\right)\right)_{\beta}(x(\theta))-\sigma^{2}\left(u^{0}(\theta, x(\theta))\right) \triangle_{x} f(x(\theta))\right) d \theta\right]\right| \\
& \leq\|\psi\|_{\infty} \sup _{k}\left\|u^{\varepsilon_{k}}\right\|_{L^{2}\left((0, T) \times \mathbb{R}^{d}\right)} \sqrt{\int_{(0, T) \times \mathbb{R}^{d}}\left|\sigma^{2}\left(u^{0}(\theta, x)\right)-\left(\sigma^{2}\left(u^{0}(\theta)\right)\right)_{\beta}(x)\right|^{2}\left|\triangle_{x} f(x)\right|^{2} d x d \theta} \\
& \leq\|\psi\|_{\infty} \sup _{k}\left\|u^{\varepsilon_{k}}\right\|_{L^{2}\left((0, T) \times \mathbb{R}^{d}\right)} \sqrt{\int_{\mathbb{R}^{d}} \phi(y) \int_{(0, T) \times \mathbb{R}^{d}}\left|\sigma^{2}\left(u^{0}(\theta, x)\right)-\sigma^{2}\left(u^{0}(\theta)\right)(x-\beta y)\right|^{2}\left|\triangle_{x} f(x)\right|^{2} d x d \theta d y}
\end{aligned}
$$

where the last upper bound tends to 0 as $k \rightarrow \infty$. We then conclude on (2.17).

\subsection{Uniqueness result for (2.1)}

Let us first start by showing that the time marginal distribution of (2.1) are unique.

Proposition 2.10. Assume that $\left(A_{0}\right),\left(A_{1}\right)$ and $\left(A_{2}\right)$ hold true. Let $\left(X_{t}^{1}, u_{t}^{1} ; t \in[0, T]\right)$ and $\left(X_{t}^{2}, u_{t}^{2} ; t \in[0, T]\right)$ be two weak solutions to (2.1). Then, for all $0 \leq t \leq T$, $u_{t}^{1}=u_{t}^{2}$ a.e. on $\mathbb{R}^{d}$. This conclusion holds true also under $\left(A_{0}\right)$, $\left(A_{1}\right)$ and $\left(A_{2}\right.$-weakened) plus the assumption that $\alpha$ is strictly increasing.

Proof. We give the proof assuming $\left(A_{0}\right),\left(A_{1}\right)$ and $\left(A_{2}\right.$-weakened) plus the assumption that $\alpha$ is strictly increasing, the other case can be easily to deduce from the following arguments. Consider two weak solutions $\left(X_{t}^{1}, u_{t}^{1} ; t \in[0, T]\right)$ and $\left(X_{t}^{2}, u_{t}^{2} ; t \in[0, T]\right)$ to $(2.1)$. Given some $\gamma>0$, that will be chosen later, define

$$
G_{s, t}^{\gamma}(x, y)=G_{t-s}^{\gamma}(x-y)=\left(2 \pi \gamma^{2}(t-s)\right)^{-\frac{d}{2}} \exp \left(-\frac{|x-y|^{2}}{2 \gamma^{2}(t-s)}\right) .
$$

Since the kernel $G_{t}^{\gamma}$ gives the fundamental solution related to the parabolic operator $\partial_{s}+\frac{\gamma^{2}}{2} \triangle_{x}$; that is

$$
\partial_{s} G_{s, t}^{\gamma}+\frac{\gamma^{2}}{2} \triangle_{x} G_{s, t}^{\gamma}=0, \lim _{s \rightarrow t^{-}} G_{s, t}^{\gamma}=\delta_{\{y\}}, \delta_{\{y\}} \text { the Dirac measure in } y,
$$

the function $v(s, x)=G_{s, t}^{\gamma}(f)(x)=G_{t-s}^{\gamma} * f(x), s \leq t, x \in \mathbb{R}^{d}, f \in \mathcal{C}_{c}^{\infty}\left(\mathbb{R}^{d}\right)$ (* denoting the convolution product on $\left.\mathbb{R}^{d}\right)$ is a $\mathcal{C}^{\infty}\left([0, t] \times \mathbb{R}^{d}\right)$ function satisfying:

$$
\left\{\begin{array}{l}
\partial_{s} v(s, x)+\frac{\gamma^{2}}{2} \triangle_{x} v(s, x)=0,0 \leq s<t, x \in \mathbb{R}^{d} \\
v(t, x)=f(x), x \in \mathbb{R}^{d}
\end{array}\right.
$$

Applying Itô formula to $v\left(s, X_{s}^{i}\right)$ for $i=1,2$,

$$
\begin{aligned}
\int f u_{t}^{i} d x=\mathbb{E}\left[v\left(t, X_{t}^{i}\right)\right] & =\mathbb{E}\left[v\left(0, X_{0}\right)\right]+\mathbb{E}\left[\int_{0}^{t} \frac{\partial v}{\partial s}\left(s, X_{s}^{i}\right) d s\right]+\frac{1}{2} \mathbb{E}\left[\int_{0}^{t} \sigma^{2}\left(u_{s}^{i}\left(X_{s}^{i}\right)\right) \triangle_{x} v\left(s, X_{s}^{i}\right) d s\right] \\
& =\mathbb{E}\left[v\left(0, X_{0}\right)\right]+\frac{1}{2} \mathbb{E}\left[\int_{0}^{t}\left(\sigma^{2}\left(u_{s}^{i}\left(X_{s}^{i}\right)\right)-\gamma^{2}\right) \triangle_{x} v\left(s, X_{s}^{i}\right) d s\right]
\end{aligned}
$$

so that for $f \in \mathcal{C}_{c}^{\infty}\left(\mathbb{R}^{d}\right), G_{0, t}^{\gamma}=G_{t}^{\gamma}$,

$$
\int f u_{t}^{i} d x=\int f\left(G_{t}^{\gamma}\left(\mu_{0}\right)+\frac{1}{2} \sum_{k=1}^{d} \int_{0}^{t} \frac{\partial^{2}}{\partial x_{k}^{2}} G_{t-s}^{\gamma}\left(\left(\sigma^{2}\left(u_{s}^{i}\right)-\gamma^{2}\right) u_{s}^{i}\right) d s\right) d x .
$$

We then obtain that

$$
\int f\left(u_{t}^{1}-u_{t}^{2}\right) d x=\int f\left(\frac{1}{2} \sum_{k=1}^{d} \int_{0}^{t} \frac{\partial^{2}}{\partial x_{k}^{2}} G_{t-s}^{\gamma}\left(\left(\sigma^{2}\left(u_{s}^{1}\right)-\gamma^{2}\right) u_{s}^{1}-\left(\sigma^{2}\left(u_{s}^{2}\right)-\gamma^{2}\right) u_{s}^{2}\right) d s\right) d x .
$$


Next, we take the supremum over all $f \in \mathcal{C}_{c}^{\infty}\left(\mathbb{R}^{d}\right)$ such that $\|f\|_{L^{2}\left(\mathbb{R}^{d}\right)}=1$ and we integrate the resulting expression over $(0, T)$. It follows that

$$
\left\|u^{1}-u^{2}\right\|_{L^{2}\left((0, T) \times \mathbb{R}^{d}\right)}=\left\|\frac{1}{2} \sum_{k=1}^{d} \int_{0} \frac{\partial^{2}}{\partial x_{k}^{2}} G_{\cdot-s}^{\gamma}\left(\left(\sigma^{2}\left(u_{s}^{1}\right)-\gamma^{2}\right) u_{s}^{1}-\left(\sigma^{2}\left(u_{s}^{2}\right)-\gamma^{2}\right) u_{s}^{2}\right) d s\right\|_{L^{2}\left((0, T) \times \mathbb{R}^{d}\right)} .
$$

Now, let us recall that for all $f \in L^{2}\left((0, T) \times \mathbb{R}^{d}\right)$, (see e.g. Stroock and Varadhan [31], Appendix A.2, Lemmas A.2.1 and A.2.2)

$$
\left\|\int_{0}^{\cdot} \partial_{x_{k} x_{l}}^{2} G_{.-s}^{\gamma}(f(s)) d s\right\|_{L^{2}\left((0, T) \times \mathbb{R}^{d}\right)} \leq \frac{2}{\gamma^{2}}\|f\|_{L^{2}\left((0, T) \times \mathbb{R}^{d}\right)}, 1 \leq k, l \leq d .
$$

Thank to the boundedness of $\sigma^{2}\left(u^{i}\right), i=1,2$, the preceding estimate ensures that the r.h.s. of (2.20) is well defined. A closer investigation of the proof arguments in [31] enables to slightly improve the preceding estimate with

$$
\left\|\int_{0} \triangle_{x} G_{\cdot-s}^{\gamma}(f(s)) d s\right\|_{L^{2}\left((0, T) \times \mathbb{R}^{d}\right)}=\left\|\sum_{k=1}^{d} \int_{0}^{\cdot} \frac{\partial^{2}}{\partial x_{k}^{2}} G_{\cdot-s}^{\gamma}(f(s)) d s\right\|_{L^{2}\left((0, T) \times \mathbb{R}^{d}\right)} \leq \frac{2}{\gamma^{2}}\|f\|_{L^{2}\left((0, T) \times \mathbb{R}^{d}\right)}
$$

(see the appendix section, for a short proof). Applying this estimate to (2.20), we obtain

$$
\begin{aligned}
\left\|u^{1}-u^{2}\right\|_{L^{2}\left((0, T) \times \mathbb{R}^{d}\right)} \leq & \frac{1}{\gamma^{2}}\left\|\left(\sigma^{2}\left(u^{1}\right)-\gamma^{2}\right) u^{1}-\left(\sigma^{2}\left(u^{2}\right)-\gamma^{2}\right) u^{2}\right\|_{L^{2}\left((0, T) \times \mathbb{R}^{d}\right)} \\
& =\frac{1}{\gamma^{2}}\left\|\left(\sigma^{2}\left(u^{1}\right) u^{1}-\sigma^{2}\left(u^{2}\right) u^{2}-\gamma^{2}\left(u^{1}-u^{2}\right)\right)\right\|_{L^{2}\left((0, T) \times \mathbb{R}^{d}\right)} .
\end{aligned}
$$

For $\alpha(r)$ as in (2.2), by observing that the first order Taylor expansion writes

$$
\sigma^{2}\left(r_{2}\right) r_{2}-\sigma^{2}\left(r_{1}\right) r_{1}=\int_{0}^{1}\left(r_{1}-r_{2}\right) \alpha\left(r_{1}+\theta\left(r_{2}-r_{1}\right)\right) d \theta
$$

and choosing $\gamma>0$ so that

$$
\gamma^{2}>\max _{i=1,2}\left|\int_{0}^{1} \alpha\left(u^{i}+\theta\left(u^{i}-u^{j}\right)\right) d \theta\right|
$$

we get

$$
\left|\left(\sigma^{2}\left(u^{1}\right) u^{1}-\sigma^{2}\left(u^{2}\right) u^{2}-\gamma^{2}\left(u^{1}-u^{2}\right)\right)\right|=\left(\gamma^{2}-\int_{0}^{1} \alpha\left(u_{1}+\theta\left(u_{2}-u_{1}\right)\right) d \theta\right)\left|u^{1}-u^{2}\right|,
$$

and deduce that

$$
\left\|u^{1}-u^{2}\right\|_{L^{2}\left((0, T) \times \mathbb{R}^{d}\right)}^{2} \leq \frac{1}{\gamma^{4}}\left\|\left(\gamma^{2}-\int_{0}^{1} \alpha\left(u_{1}+\theta\left(u_{2}-u_{1}\right)\right) d \theta\right)\left|u^{1}-u^{2}\right|\right\|_{L^{2}\left((0, T) \times \mathbb{R}^{d}\right)}^{2} .
$$

Splitting $\left\|u^{1}-u^{2}\right\|_{L^{2}\left((0, T) \times \mathbb{R}^{d}\right)}^{2}$ into the sum

$$
\left\|\left(u^{1}-u^{2}\right) \mathbb{1}_{\left\{u^{2}-u^{1} \geq \kappa\right\}}\right\|_{L^{2}\left((0, T) \times \mathbb{R}^{d}\right)}^{2}+\left\|\left(u^{1}-u^{2}\right) \mathbb{1}_{\left\{u^{2}-u^{1}<\kappa\right\}}\right\|_{L^{2}\left((0, T) \times \mathbb{R}^{d}\right)}^{2}
$$

for some arbitrary $\kappa>0,(2.22)$ reduces to

$$
\begin{aligned}
& \left\|\left(u^{1}-u^{2}\right) \mathbb{1}_{\left\{u^{2}-u^{1} \geq \kappa\right\}}\right\|_{L^{2}\left((0, T) \times \mathbb{R}^{d}\right)}^{2} \\
& \leq \frac{1}{\gamma^{4}}\left\|\left(\gamma^{2}-\int_{0}^{1} \alpha\left(u_{1}+\theta\left(u_{2}-u_{1}\right)\right) d \theta\right)\left|u^{1}-u^{2}\right|\right\|_{L^{2}\left((0, T) \times \mathbb{R}^{d}\right)}^{2}-\left\|\left(u^{1}-u^{2}\right) \mathbb{1}_{\left\{u^{2}-u^{1}<\kappa\right\}}\right\|_{L^{2}\left((0, T) \times \mathbb{R}^{d}\right)}^{2} \\
& \leq \frac{1}{\gamma^{4}}\left\|\left(\gamma^{2}-\int_{0}^{1} \alpha\left(u_{1}+\theta\left(u_{2}-u_{1}\right)\right) d \theta\right)\left|u^{1}-u^{2}\right| \mathbb{1}_{\left\{u^{2}-u^{1} \geq \kappa\right\}}\right\|_{L^{2}\left((0, T) \times \mathbb{R}^{d}\right)}^{2}
\end{aligned}
$$


Fixing $\kappa>0$, and setting

$$
\zeta^{2}(\kappa):=\sup \left\{\beta>0 ; \int_{0}^{1} \alpha(r \kappa) d r>\beta\right\}
$$

which is (strictly) positive by ( $A_{2}$-weakened) and the monotone assumption of $\alpha$,

$$
\gamma^{2}-\int_{0}^{1} \alpha\left(u_{1}+\theta\left(u_{2}-u_{1}\right)\right) d \theta \leq \gamma^{2}-\int_{0}^{1} \alpha(\theta \kappa) d \theta \leq \gamma^{2}-\zeta^{2}(\kappa)
$$

which implies that $\left\|\left|u^{1}-u^{2}\right| \mathbb{1}_{\left\{u^{2}-u^{1} \geq \kappa\right\}}\right\|_{L^{2}\left((0, T) \times \mathbb{R}^{d}\right)}<\left(\gamma^{2}-\zeta^{2}\right)\left\|\left|u^{1}-u^{2}\right| \mathbb{1}_{\left\{u^{2}-u^{1} \geq \kappa\right\}}\right\|_{L^{2}\left((0, T) \times \mathbb{R}^{d}\right)} / \gamma^{2}<$ $\left\|\left|u^{1}-u^{2}\right| \mathbb{1}_{\left\{u^{2}-u^{1} \geq \kappa\right\}}\right\|_{L^{2}\left((0, T) \times \mathbb{R}^{d}\right)}$. Since $\kappa$ is arbitrary, $u_{2} \leq u_{1}$ for a.e. on $(0, T) \times \mathbb{R}^{d}$. By symmetry, we can also exchange $u^{1}$ and $u^{2}$, and deduce that $u_{1} \leq u_{2}$ for a.e. on $(0, T) \times \mathbb{R}^{d}$, from which we conclude that $u_{1}=u_{2}$ for a.a. $(t, x) \in(0, T) \times \mathbb{R}^{d}$. This conclude the claim.

To conclude on the strong uniqueness of the solution to (2.8), let us recall the following result due to Champagnat and Jabin [8].

Theorem 2.11 (Theorems 1.1 and 1.2, [8]). Let $\left(Z_{t}^{1} ; t \geq 0\right)$ and $\left(Z_{t}^{2} ; t \geq 0\right)$ be two solutions to the SDE

$$
d Z_{t}=\Sigma\left(t, Z_{t}\right) d W_{t}, Z_{0}=\xi
$$

with one-dimensional time marginal $u_{Z_{1}}(t, z) d z$ and $u_{Z_{2}}(t, z) d z$ in $L_{\text {loc }}^{2 q}\left((0, \infty) ; W^{1,2 p}\left(\mathbb{R}^{d}\right)\right)$. If $\Sigma:(0, \infty) \times \mathbb{R}^{d} \rightarrow$ $\mathbb{R}^{d \times d}$ is in $L^{\infty}\left((0, \infty) \times \mathbb{R}^{d}\right) \cap L_{\text {loc }}^{q^{\prime}}\left((0, \infty) ; W^{1, p^{\prime}}\left(\mathbb{R}^{d}\right)\right)$, for $1 / p+1 / p^{\prime}=1,1 / q+1 / q^{\prime}=1$, then one has pathwise uniqueness: a.s. $\sup _{t \geq 0}\left|Z_{t}^{1}-Z_{t}^{2}\right|=0$.

According to Proposition 2.10 and Corollary 2.9, for any solution to (2.1), $u$ is in $L^{\infty}\left((0, T) \times \mathbb{R}^{d}\right)$. Hence, a direct application of Theorem 2.11 (with $p=q=1, p^{\prime}=q^{\prime}=\infty$ ) gives

Proposition 2.12. Under $\left(A_{0}\right),\left(A_{1}\right)$ and $\left(A_{2}\right),(2.1)$ admits at most one strong solution.

\subsection{Generalization to matrix valued diffusion}

We may remark that the main ideas for the proof of Theorem 2.3 can be extended to obtain the existence and uniqueness of a weak solution to (2.1) in the situation where the diffusion component is a $d \times d$-matrix valued function; namely $\sigma:[0, \infty) \rightarrow \mathbb{R}^{d \times d}$. Such extension holds provided that the assumption $\left(A_{0}\right)$ remains unchanged meanwhile $\left(A_{1}\right)$, $\left(A_{2}\right)$ and $\left(A_{2}\right.$-weakened) are respectively replaced by the following:

\section{Hypothesis 2.13.}

$\left(\boldsymbol{A}_{1}^{\prime}\right)$ The map $r \mapsto \sigma(r) \in \mathbb{R}^{d \times d}$ is continuously differentiable on $\mathbb{R}^{+}$.

$\left(\boldsymbol{A}_{2}^{\prime}\right)$ For $a(r)=\sigma \sigma^{*}(r)$, the map $r \in[0, \infty) \mapsto \alpha(r) \in \mathbb{R}^{d \times d}$ given by

$$
\alpha(r)=\left\{\alpha^{i, j}(r):=\left(a^{i, j}(r) r\right)^{\prime}=\left(a^{i, j}\right)^{\prime}(r) r+a^{i, j}(r), 1 \leq i, j \leq d\right\},
$$

is continuous and strongly elliptic in the sense that, for some $\eta_{a}>0$,

$$
\xi \cdot \alpha(r) \xi \geq \eta_{a}|\xi|^{2}, \forall r \geq 0, \forall \xi \in \mathbb{R}^{d} .
$$

( $\boldsymbol{A}_{2}^{\prime}$-weakened) For $a(r)=\sigma \sigma^{*}(r)$, the map $r \in[0, \infty) \mapsto \alpha(r) \in \mathbb{R}^{d} \times \mathbb{R}^{d}$ given by

$$
\alpha(r)=\left\{\alpha^{i, j}(r):=\left(a^{i, j}(r) r\right)^{\prime}=\left(a^{i, j}\right)^{\prime}(r) r+a^{i, j}(r), 1 \leq i, j \leq d\right\}
$$

is continuous and positive semi-definite:

$$
\xi \cdot \alpha(r) \xi \geq 0, \forall \xi \in \mathbb{R}^{d}
$$


The particular strict monotone assumption in Proposition 2.10 can be replaced by the assumption that

$$
\xi \cdot \alpha(r) \xi<\xi \cdot \alpha\left(r^{\prime}\right) \xi, \forall \xi \in \mathbb{R}^{d}, \forall r, r^{\prime} \geq 0 \text { such that } r<r^{\prime} .
$$

Under $\left(A_{0}\right),\left(A_{1}^{\prime}\right)$ and $\left(A_{2}^{\prime}\right)$, Theorem 2.3 can be extended to the existence and uniqueness of a strong solution to

$$
\left\{\begin{array}{l}
X_{t}=X_{0}+\int_{0}^{t} \sigma\left(u\left(s, X_{s}\right)\right) d W_{s}, \quad 0 \leq t \leq T, \\
d \operatorname{Law}\left(X_{t}\right)=u(t, x) d x \text { with } u \in L^{\infty}\left((0, T) \times \mathbb{R}^{d}\right) \cap L^{2}\left((0, T) \times \mathbb{R}^{d}\right), \\
u(0, x)=u_{0}(x), x \in \mathbb{R}^{d}, \sigma: \mathbb{R}^{+} \rightarrow \mathbb{R}^{d \times d}
\end{array}\right.
$$

\section{For the existence of a weak solution to (2.24)}

Assumptions $\left(A_{0}\right),\left(A_{1}^{\prime}\right)$ and $\left(A_{2}^{\prime}\right)$ are enough to replicate the proof arguments of the estimates 2.10 and 2.11 in Lemma 2.5 and enables to construct, as in Proposition 2.6, a weak solution to

$$
\left\{\begin{array}{l}
X_{t}^{\varepsilon}=X_{0}+\int_{0}^{t} \sqrt{a_{\varepsilon}\left(u^{\varepsilon}\left(s, X_{s}\right)\right)} d W_{s}, \quad 0 \leq t \leq T \\
d \operatorname{Law}\left(X_{t}^{\varepsilon}\right)=u^{\varepsilon}(t, x) d x, \text { with } u^{\varepsilon} \in L^{\infty}\left((0, T) \times \mathbb{R}^{d}\right) \cap L^{2}\left((0, T) \times \mathbb{R}^{d}\right), \\
u^{\varepsilon}(0, x)=u_{0}(x), \sigma: \mathbb{R}^{+} \rightarrow \mathbb{R}^{d} \times \mathbb{R}^{d}
\end{array}\right.
$$

where $\sqrt{a_{\varepsilon}(r)}$ is the square root matrix of $a(r)+\varepsilon I_{d}, a^{\prime}(r)=\left\{\left(a^{i, j}\right)^{\prime}(r), 1 \leq i, j \leq d\right\}$. Although the identity (2.12) doesn't have any trivial multidimensional extension, and since $u^{\varepsilon}$ satisfies the analogous of (2.7): for all for all $0 \leq T_{0} \leq T, f \in \mathcal{C}_{c}^{1,2}\left(\left[0, T_{0}\right] \times \mathbb{R}^{d}\right)$,

$$
\begin{aligned}
& \int_{\mathbb{R}^{d}} u^{\varepsilon}\left(T_{0}, x\right) f\left(T_{0}, x\right) d x-\int_{\mathbb{R}^{d}} u_{0}(x) f(0, x) d x \\
& =\int_{\left(0, T_{0}\right) \times \mathbb{R}^{d}} u^{\varepsilon}(t, x) \partial_{t} f(t, x)-\nabla_{x} u^{\varepsilon}(t, x) \cdot a_{\varepsilon}^{\prime}\left(u^{\varepsilon}(t, x)\right) \nabla_{x} f(t, x) d t d x,
\end{aligned}
$$

the convergence (up to a subsequence) of $\left\{\left(X_{t}^{\varepsilon}, u^{\varepsilon}(t) ; 0 \leq t \leq T\right)\right\}_{\varepsilon>0}$ to a weak solution to (2.24) can still be derived from the energy estimate:

$$
\max _{0 \leq t \leq T}\left\|u^{\varepsilon}(t)\right\|_{L^{2}\left(\mathbb{R}^{d}\right)}^{2}+\eta_{a} \int_{0}^{T}\left\|\nabla_{x} u^{\varepsilon}(t)\right\|_{L^{2}\left(\mathbb{R}^{d}\right)}^{2} d t \leq\left\|u_{0}\right\|_{L^{2}\left(\mathbb{R}^{d}\right)}^{2}
$$

which follows from (2.25).

\section{For the uniqueness of a strong solution to (2.24)}

$\operatorname{Under}\left(A_{1}^{\prime}\right)$ and $\left(A_{2}^{\prime}\right.$-weakened), the main arguments of Proposition 2.10 can be extended, replacing $G_{s, t}^{\gamma}$ by the fundamental solution to $G_{s, t}^{\Gamma}$ related to the parabolic operator $L(f)=\partial_{s} f+\frac{1}{2} \operatorname{Trace}\left(\Gamma \nabla_{x}^{2} f\right)$ where $\Gamma$ is a (constant) positive definite matrix such that $\xi \cdot \Gamma \xi \geq \gamma^{2}|\xi|^{2}, \gamma \neq 0$. Taking two weak solutions $\left(X_{t}^{1}, u_{t}^{1} ; t \in[0, T]\right)$ and $\left(X_{t}^{2}, u_{t}^{2} ; t \in[0, T]\right)$ to $(2.25)$, and by replicating the first proof steps of Proposition 2.10, we get the analog of (2.20):

$$
\left\|u^{1}-u^{2}\right\|_{L^{2}\left((0, T) \times \mathbb{R}^{d}\right)}=\left\|\frac{1}{2} \sum_{k, l=1}^{d} \int_{0} \partial_{x_{k} x_{l}}^{2} G_{.-s}^{\gamma}\left(\left(a\left(u_{s}^{1}\right)-\Gamma\right)^{k, l} u_{s}^{1}-\left(a\left(u_{s}^{2}\right)-\Gamma\right)^{k, l} u_{s}^{2}\right) d s\right\|_{L^{2}\left((0, T) \times \mathbb{R}^{d}\right)} .
$$

Using the following generalization of (2.21) (see Appendix section):

$$
\left\|\sum_{i, j=1}^{d} \int_{0} \partial_{x_{i} x_{j}}^{2} G_{.-s}^{\Gamma}\left(F^{i, j}(s)\right) d s\right\|_{L^{2}\left((0, T) \times \mathbb{R}^{d}\right)} \leq \frac{2}{\gamma^{2}} \sum_{i, j=1}^{d}\left\|F^{i, j}\right\|_{L^{2}\left((0, T) \times \mathbb{R}^{d}\right)}, \quad F^{i, j} \in L^{2}\left((0, T) \times \mathbb{R}^{d}\right),
$$


we deduce the analog of (2.22)

$$
\left\|u^{1}-u^{2}\right\|_{L^{2}\left((0, T) \times \mathbb{R}^{d}\right)} \leq \frac{1}{\gamma^{2}} \sum_{k, l=1}^{d}\left\|\left(\Gamma-\int_{0}^{1} \alpha\left(u_{1}+\theta\left(u_{2}-u_{1}\right)\right) d \theta\right)^{k, l}\left|u^{1}-u^{2}\right|\right\|_{L^{2}\left((0, T) \times \mathbb{R}^{d}\right)} .
$$

Taking $\Gamma$ large enough so that $\xi \cdot(\Gamma-\alpha(r)) \xi<0$, for all $\xi \in \mathbb{R}^{d}, r \geq 0$, the strict elliptic assumption in $\left(A_{2}^{\prime}\right)$ or (2.23) are sufficient to ensure that $u^{1}=u^{2}$. The uniqueness of a strong solution to (2.24) still follows from Champagnat and Jabin [8].

\section{Conditional nonlinear diffusion case}

\section{Hypothesis 3.1.}

(H0) The initial law $\mu_{0}$ admits a density $\rho_{0}$ such that $\int_{\mathbb{R}^{d} \times \mathbb{R}^{d}}\left(|x|^{2}+|y|^{2}\right) \rho_{0}(x, y) d x d y<\infty$.

(H1) The coefficients $b$ and $\sigma$ are bounded Lipschitz continuous functions

(H2) The kernels $\ell$ and $\gamma$ are bounded and Lipschitz continuous on $\mathbb{R}^{d}$.

(H3) Strong ellipticity is assumed for $\sigma$ : there exists $a_{*}>0$ such that, for all $x \in \mathbb{R}^{d}$,

$$
a_{*}|\xi|^{2} \leq \xi \sigma(x) \xi, \quad \forall \xi \in \mathbb{R}^{d} .
$$

\section{Hypothesis 3.2.}

(H4) The initial marginal density $\rho_{X}(0, x)=\int_{\mathbb{R}^{d}} \rho_{0}(x, y) d y$ is in $L^{1}\left(\mathbb{R}^{d}\right) \cap L^{p}\left(\mathbb{R}^{d}\right)$ for some $p \geq 2 d+2$. Moreover, for all $R>0$, for all $x \in B(0, R)$, there exists a constant $\mu_{R}>0$ such that $\rho_{X}(0, x) \geq \mu_{R}$.

(H5) $\sigma$ and $\gamma$ are in $\mathcal{C}^{2}\left(\mathbb{R}^{d}\right)$ with bounded derivatives up to second order.

(H6) Strong ellipticity is assumed for $\gamma \gamma^{*}$ : there exists $\alpha_{*}>0$ such that, for all $(x, y) \in \mathbb{R}^{d} \times \mathbb{R}^{d}$,

$$
\alpha_{*}|\xi|^{2} \leq \xi \gamma(y) \gamma(y)^{*} \xi, \quad \forall \xi \in \mathbb{R}^{d} .
$$

Our main result concerns the wellposedness (in the weak and strong sense) of a solution to (1.2). More precisely, we have

Theorem 3.3. Under Hypothesis 3.1, there exists a unique weak solution to (1.2). With the addition of Hypothesis 3.2, pathwise uniqueness holds for the solution of (1.2) and $\operatorname{Law}\left(X_{t}, Y_{t}\right)$ admits a density function at all time $0 \leq t \leq T$.

Before entering in the details of the proof, let us point out an important remark for the construction of the solution of (1.2). Consider for a while the case when $\left(X_{t} ; t \geq 0\right)$ doesn't depend on $\left(Y_{t} ; t \geq 0\right)$, namely when $b$ does not depend on $Y$, or the simpler situation when $b=0$. Hypothesis 3.1 ensure the existence of a unique strong solution for

$$
X_{t}=X_{0}+\int_{0}^{t} \sigma\left(X_{s}\right) d B_{s} .
$$

Then, based on the fact that $\left(X_{t} ; t \geq 0\right)$ is now an exogenous process, we can consider the following fixed point construction, similar to those in Sznitman [33] and Méléard [24]. From now on, we fix an arbitrary time horizon $0 \leq T<+\infty$ and we consider the classical Hilbert space $M^{2}(0, T)$ of real adapted continuous processes $\zeta=\left(\zeta_{t} ; 0 \leq\right.$ $t \leq T)$ such that $\mathbb{E}_{\mathbb{P}}\left[\int_{0}^{T} \zeta_{s}^{2} d s\right]<+\infty\left(\mathbb{E}_{\mathbb{P}}\right.$ denoting the expectation under $\left.\mathbb{P}\right)$, and endowed with the following scalar product and norm

$$
(\zeta, \xi)_{c}=\mathbb{E}_{\mathbb{P}}\left[\int_{0}^{T} \exp (-c s) \zeta_{s} \xi_{s} d s\right], \quad\|\zeta\|_{c}^{2}=\mathbb{E}_{\mathbb{P}}\left[\int_{0}^{T} \exp (-c s)\left|\zeta_{s}\right|^{2} d s\right],
$$


where $c$ is a positive constant that will be chosen later. Given $\left(\Omega, \mathcal{F},\left(\mathcal{F}_{t} ; 0 \leq t \leq T\right), \mathbb{P}\right)$ a filtered probability space under which are defined $\left(W_{t} ; 0 \leq t \leq T\right)$ and $\left(B_{t} ; 0 \leq t \leq T\right)$, two independent $\mathbb{R}^{d}$-Brownian motions, and $\left(X_{0}, Y_{0}\right) \sim \mu_{0}$ (independent of $\left(W_{t} ; 0 \leq t \leq T\right)$ and $\left(B_{t} ; 0 \leq t \leq T\right)$ ).

From any element $\zeta$ in $M^{2}(0, T)$, we construct the application $\zeta \mapsto Y(\zeta)$ taking values in $M^{2}(0, T)$ and defined as

$$
Y(\zeta)_{t}=Y_{0}+\int_{0}^{t} \mathbb{E}_{\mathbb{P}}\left[\ell\left(\zeta_{s}\right) \mid X_{s}\right] d s+\int_{0}^{t} \mathbb{E}_{\mathbb{P}}\left[\gamma\left(\zeta_{s}\right) \mid X_{s}\right] d W_{s}, 0 \leq t \leq T .
$$

Owing to (H0) and (H2), it is clear that $\|Y(\zeta)\|_{c}<+\infty$. Now, for $\zeta$ and $\xi$ in $M^{2}(0, T), 0 \leq t \leq T$, we have

$$
\begin{aligned}
\mathbb{E}_{\mathbb{P}}\left[\left|Y(\zeta)_{t}-Y(\xi)_{t}\right|^{2}\right] \leq & 2 \int_{0}^{t} \mathbb{E}_{\mathbb{P}}\left[\left|\mathbb{E}_{\mathbb{P}}\left[\ell\left(\zeta_{s}\right) \mid X_{s}\right]-\mathbb{E}_{\mathbb{P}}\left[\ell\left(\xi_{s}\right) \mid X_{s}\right]\right|^{2}\right] d s \\
& +2 \int_{0}^{t} \mathbb{E}_{\mathbb{P}}\left[\left\|\mathbb{E}_{\mathbb{P}}\left[\gamma\left(\zeta_{s}\right) \mid X_{s}\right]-\mathbb{E}_{\mathbb{P}}\left[\gamma\left(\xi_{s}\right) \mid X_{s}\right]\right\|^{2}\right] d s \\
\leq & 2\left(\|\ell\|_{\text {Lip }}^{2}+\|\gamma\|_{\text {Lip }}^{2}\right) \int_{0}^{t} \mathbb{E}_{\mathbb{P}}\left[\mathbb{E}_{\mathbb{P}}\left[\left|\zeta_{s}-\xi_{s}\right|^{2} \mid X_{s}\right]\right] d s \\
\leq & 2\left(\|\ell\|_{\text {Lip }}^{2}+\|\gamma\|_{\text {Lip }}^{2}\right) \int_{0}^{t} \mathbb{E}_{\mathbb{P}}\left[\left|\zeta_{s}-\xi_{s}\right|^{2}\right] d s .
\end{aligned}
$$

Multiplying both sides by $\exp (-c t)$ and integrating (in time) the resulting expression over the interval $(0, T)$ gives

$$
\int_{0}^{T} \exp (-c t) \mathbb{E}_{\mathbb{P}}\left[\left|Y(\zeta)_{t}-Y(\xi)_{t}\right|^{2}\right] d t \leq 2\left(\|\ell\|_{L i p}^{2}+\|\gamma\|_{L i p}^{2}\right) \int_{0}^{T} \exp (-c t) \int_{0}^{t} \mathbb{E}_{\mathbb{P}}\left[\left|\zeta_{s}-\xi_{s}\right|^{2}\right] d s d t
$$

An integration by part in time then yields

$$
\begin{aligned}
\|Y(\zeta)-Y(\xi)\|_{c}^{2} & \leq-\frac{\exp (-c T)}{c} \int_{0}^{T} \mathbb{E}_{\mathbb{P}}\left[\left|\zeta_{s}-\xi_{s}\right|^{2}\right] d s+\frac{2}{c}\left(\|\ell\|_{L i p}^{2}+\|\gamma\|_{L i p}^{2}\right) \int_{0}^{T} \exp (-c t) \mathbb{E}_{\mathbb{P}}\left[\left|\zeta_{t}-\xi_{t}\right|^{2}\right] d t \\
& \leq \frac{2}{c}\left(\|\ell\|_{\text {Lip }}^{2}+\|\gamma\|_{\text {Lip }}^{2}\right)\|\zeta-\xi\|_{c}^{2} .
\end{aligned}
$$

Choosing $c>2\left(\|\ell\|_{L i p}^{2}+\|\gamma\|_{L i p}^{2}\right)$, we get the existence of a unique fixed point solution of equation (1.2), when $b=0$. In the case $b(x, y)=b(x)$, the same arguments lead to the same result.

The rest of this section is dedicated to the proof of Theorem 3.3, which essentially relies on a Girsanov transform to go back to a situation similar to the previous case. First, in Section 3.1, we collect some preliminary remarks on the Girsanov transform that we use to remove the drift in (1.2), and deduce some apriori controls on the associated change of probability measure. (1.2).

Second, in Section 3.2, we use the fixed point technique for the $L^{2}$-existence and weak uniqueness for solution to

Finally in Section 3.3, assuming some stronger regularity on the kernels $\ell$ and $\gamma$ and strong ellipticity on $\gamma$ (see Hypothesis 3.2, we obtain some apriori regularity on the nonlinear coefficients using averaging lemma technique, and then deduce the strong uniqueness property.

\subsection{Preliminary remarks on (1.2)}

Fix an arbitrary $0 \leq T<+\infty$ and let $\left(X_{t}, Y_{t} ; 0 \leq t \leq T\right)$ be a solution to (1.2) up to $T$, defined on $\left(\Omega, \mathcal{F},\left(\mathcal{F}_{t} ; 0 \leq\right.\right.$ $t \leq T), \mathbb{P})$. Then, define $\left(Z_{t} ; 0 \leq t \leq T\right)$ as

$$
Z_{t}=\exp \left\{-\int_{0}^{t}\left(\sigma^{-1} b\right)\left(X_{s}, Y_{s}\right) d B_{s}-\frac{1}{2} \int_{0}^{t}\left|\sigma^{-1} b\right|^{2}\left(X_{s}, Y_{s}\right) d s\right\}
$$


for $\sigma^{-1}(x)$ the inverse matrix of $\sigma(x),\left(\sigma^{-1} b\right)(x, y)=\sigma^{-1}(x) b(x, y)$ and $\left|\sigma^{-1} b\right|^{2}(x, y)=\left(\sigma^{-1} b\right)(x, y) \cdot\left(\sigma^{-1} b\right)(x, y)$. Then, under the probability measure $\mathbb{Q}$ defined on $\left(\Omega, \mathcal{F}_{T},\left(\mathcal{F}_{t} ; 0 \leq t \leq T\right)\right)$ by

$$
\left.\frac{d \mathbb{Q}}{d \mathbb{P}}\right|_{\mathcal{F}_{T}}=Z_{T}
$$

the process

$$
\widehat{B}_{t}=\int_{0}^{t}\left(\sigma^{-1} b\right)\left(X_{s}, Y_{s}\right) d s+B_{t}, 0 \leq t \leq T,
$$

is a $\mathbb{R}^{d}$-Brownian motion (by means of Girsanov transformation). Observing that the covariation between $\left(W_{t} ; 0 \leq\right.$ $t \leq T)$ and $\left(\widehat{B}_{t} ; 0 \leq t \leq T\right)$ is zero, $\left(\widehat{B}_{t} ; 0 \leq t \leq T\right)$ is independent of $\left(W_{t} ; 0 \leq t \leq T\right)$.

In addition, for all $\mathcal{F}_{t}$-adapted process $\left(\theta_{t} ; 0 \leq t \leq T\right)$ such that $\mathbb{E}_{\mathbb{P}}\left[\left|\theta_{t}\right|\right]<+\infty$ for all $t$, the characterization of the conditional expectation ensures that, $\mathbb{P}$-a.s. (or equivalently $\mathbb{Q}$-a.s.),

$$
\mathbb{E}_{\mathbb{P}}\left[\theta_{t} \mid X_{t}\right]=Z_{t} \mathbb{E}_{\mathbb{Q}}\left[\left(Z_{t}\right)^{-1} \theta_{t} \mid X_{t}\right], 0 \leq t \leq T .
$$

Following this change of probability measure, under $\mathbb{Q}$, the equation (1.2) formulate as the following self-contained SDE:

$$
\left\{\begin{array}{l}
X_{t}=X_{0}+\int_{0}^{t} \sigma\left(X_{s}\right) d \widehat{B}_{s}, 0 \leq t \leq T \\
Y_{t}=Y_{0}+\int_{0}^{t} Z_{s} \mathbb{E}_{\mathbb{Q}}\left[Z_{s}^{-1} \ell\left(Y_{s}\right) \mid X_{s}\right] d s+\int_{0}^{t} Z_{s} \mathbb{E}_{\mathbb{Q}}\left[Z_{s}^{-1} \gamma\left(Y_{s}\right) \mid X_{s}\right] d W_{s}, \\
Z_{t}=\exp \left\{-\int_{0}^{t}\left(\sigma^{-1} b\right)\left(X_{s}, Y_{s}\right) d \widehat{B}_{s}+\frac{1}{2} \int_{0}^{t}\left|\sigma^{-1} b\right|^{2}\left(X_{s}, Y_{s}\right) d s\right\}, \\
\left(X_{0}, Y_{0}\right) \sim \mu_{0} .
\end{array}\right.
$$

Conversely, starting from (3.4), defined on $\left(\Omega, \mathcal{F}_{T},\left(\mathcal{F}_{t}, 0 \leq t \leq T\right), \mathbb{Q}\right)$ endowed with two independent Brownian motions $\left(\widehat{B}_{t} ; 0 \leq t \leq T\right)$ and $\left(W_{t} ; 0 \leq t \leq T\right)$, independent to $\left(X_{0}, Y_{0}\right)$, one can easily check that $\left(X_{t}, Y_{t} ; 0 \leq t \leq\right.$ $T)$ is a solution to $(1.2)$ on $\left(\Omega, \mathcal{F}_{T},\left(\mathcal{F}_{t}, 0 \leq t \leq T\right), \widehat{\mathbb{P}}\right)$ where $\widehat{\mathbb{P}}$ is given by

$$
\left.\frac{d \widehat{\mathbb{P}}}{d \mathbb{Q}}\right|_{\mathcal{F}_{T}}=Z_{T}^{-1}=\exp \left\{\int_{0}^{t}\left(\sigma^{-1} b\right)\left(X_{s}, Y_{s}\right) d \widehat{B}_{s}-\frac{1}{2} \int_{0}^{t}\left|\sigma^{-1} b\right|^{2}\left(X_{s}, Y_{s}\right) d s\right\}, 0 \leq t \leq T .
$$

The existence and uniqueness of a weak solution to (1.2) is then an immediate consequence of the existence and uniqueness of a weak solution to (3.4).

Let $\zeta$ in $M^{2}(0, T)$. We consider the linearized system

$$
\left\{\begin{array}{l}
X_{t}=X_{0}+\int_{0}^{t} \sigma\left(X_{s}\right) d \widehat{B}_{s}, 0 \leq t \leq T \\
Z(\zeta)_{t}=\exp \left\{-\int_{0}^{t}\left(\sigma^{-1} b\right)\left(X_{s}, \zeta_{s}\right) d \widehat{B}_{s}+\frac{1}{2} \int_{0}^{t}\left|\sigma^{-1} b\right|^{2}\left(X_{s}, \zeta_{s}\right) d s\right\} \\
\left(X_{0}, Y_{0}\right) \sim \mu_{0} .
\end{array}\right.
$$

Lemma 3.4. Assume Hypothesis 3.1. Let $\zeta$ and $\xi$ in $M^{2}(0, T)$.

(i) There exits a positive constant $C$ depending only on $T$ and on $\left\|\sigma^{-1} b\right\|_{\infty}$ such that

$$
\mathbb{E}_{\mathbb{Q}}\left[Z(\zeta)_{t}^{2} \mid X_{t}\right]\left(Z(\zeta)_{t}\right)^{-2} \leq C
$$

(ii) There exits a positive constant $C^{\prime}$ depending only on $T,\left\|\sigma^{-1} b\right\|_{\infty}$ and $\|\ell\|_{\text {Lip }}+\|\gamma\|_{\text {Lip }}$ such that

$$
\mathbb{E}_{\mathbb{Q}}\left[\max _{0 \leq s \leq t} \frac{\left|Z(\zeta)_{s}^{2}-Z(\xi)_{s}\right|^{2}}{Z(\zeta)_{s}^{2}}\right] \leq C^{\prime} \int_{0}^{t} \mathbb{E}_{\mathbb{Q}}\left[\frac{\left|Z(\zeta)_{s}-Z(\xi)_{s}\right|^{2}}{Z(\zeta)_{s}^{2}}+\left|\zeta_{s}-\xi_{s}\right|^{2}\right] d s
$$


Proof. For $(i)$, we fix $\zeta \in M^{2}(0, T)$. We denote by $\mathcal{E}$ the exponential martingale (under $\mathbb{Q}$ ) defined as

$$
\mathcal{E}_{t}=\exp \left\{-\int_{0}^{t} 2\left(\sigma^{-1} b\right)\left(X_{s}, \zeta_{s}\right) d \widehat{B}_{s}-\int_{0}^{t} 2\left|\sigma^{-1} b\right|^{2}\left(X_{s}, \zeta_{s}\right) d s\right\}
$$

From (3.5), we have

$$
Z(\zeta)_{t}^{2}=\mathcal{E}_{t} \exp \left\{+3 \int_{0}^{t}\left|\sigma^{-1} b\right|^{2}\left(X_{s}, \zeta_{s}\right) d s\right\}
$$

and then

$$
\begin{aligned}
\mathbb{E}_{\mathbb{Q}}\left[(Z(\zeta))_{t}^{2} \mid X_{t}\right]\left(Z(\zeta)_{t}\right)^{-2}= & \mathcal{E}_{t}^{-1} \mathbb{E}_{\mathbb{Q}}\left[\mathcal{E}_{t} \exp \left\{+3 \int_{0}^{t}\left|\sigma^{-1} b\right|^{2}\left(X_{s}, \zeta_{s}\right) d s\right\} \mid X_{t}\right] \exp \left\{-3 \int_{0}^{t}\left|\sigma^{-1} b\right|^{2}\left(X_{s}, \zeta_{s}\right) d s\right\} \\
& \leq \mathcal{E}_{t}^{-1} \mathbb{E}_{\mathbb{Q}}\left[\mathcal{E}_{t} \exp \left\{+3 \int_{0}^{t}\left|\sigma^{-1} b\right|^{2}\left(X_{s}, \zeta_{s}\right) d s\right\} \mid X_{t}\right] .
\end{aligned}
$$

Let us define the probability measure $\widetilde{\mathbb{Q}}$ on $\left(\Omega, \mathcal{F}_{T},\left(\mathcal{F}_{t} ; 0 \leq t \leq T\right)\right)$ by

$$
\left.\frac{d \widetilde{\mathbb{Q}}}{d \mathbb{Q}}\right|_{\mathcal{F}_{T}}=\mathcal{E}_{T} .
$$

Then, as in (3.3), we obtain that for all $\mathcal{F}_{t}$-adapted process $\left(\theta_{t} ; 0 \leq t \leq T\right)$ such that $\mathbb{E}_{\widetilde{\mathbb{Q}}}\left[\left|\theta_{t}\right|\right]<+\infty$ for all $t$ in $[0, T]$, the characterization of the conditional expectation ensures that, $\mathbb{P}$-a.s. (or equivalently $\mathbb{Q}$-a.s.),

$$
\mathbb{E}_{\widetilde{\mathbb{Q}}}\left[\theta_{t} \mid X_{t}\right]=\mathcal{E}_{t}^{-1} \mathbb{E}_{\mathbb{Q}}\left[\mathcal{E}_{t} \theta_{t} \mid X_{t}\right], 0 \leq t \leq T,
$$

from which we immediately deduce that

$$
\mathcal{E}_{t}^{-1} \mathbb{E}_{\mathbb{Q}}\left[\mathcal{E}_{t} \exp \left\{+3 \int_{0}^{t}\left|\sigma^{-1} b\right|^{2}\left(X_{s}, \zeta_{s}\right) d s\right\} \mid X_{t}\right] \leq \exp \left(3 T\left\|\sigma^{-1} b\right\|_{\infty}\right) .
$$

For (ii), we fix again a $\zeta \in M^{2}(0, T)$. From (3.5), we have

$$
d Z(\zeta)_{t}=Z(\zeta)_{t}\left(-\left(\sigma^{-1} b\right)\left(X_{t}, \zeta_{t}\right) d \widehat{B}_{t}+\left|\sigma^{-1} b\right|^{2}\left(X_{t}, \zeta_{t}\right) d t\right), Z(\zeta)_{0}=1,
$$

and

$$
d Z(\zeta)_{t}^{-1}=Z(\zeta)_{t}^{-1}\left(\sigma^{-1} b\right)\left(X_{t}, \zeta_{t}\right) d \widehat{B}_{t}, Z(\zeta)_{0}=1,
$$

from which we compute, using the Itô formula, for a $\xi \in M^{2}(0, T)$

$$
\begin{aligned}
& \left|Z(\zeta)_{t}-Z(\xi)_{t}\right|^{2} \\
& =2 \int_{0}^{t}\left(Z(\zeta)_{s}-Z(\xi)_{s}\right)\left\{Z(\zeta)_{s}\left(\sigma^{-1} b\right)\left(X_{s}, \zeta_{s}\right)-Z(\xi)_{s}\left(\sigma^{-1} b\right)\left(X_{s}, \xi_{s}\right)\right\} d \widehat{B}_{s} \\
& \quad+2 \int_{0}^{t}\left(Z(\zeta)_{s}-Z(\xi)_{s}\right)\left\{Z(\zeta)_{s}\left|\sigma^{-1} b\right|^{2}\left(X_{s}, \zeta_{s}\right)-Z(\xi)_{s}\left|\sigma^{-1} b\right|^{2}\left(X_{s}, \xi_{s}\right)\right\} d s \\
& \quad+\int_{0}^{t}\left(Z(\zeta)_{s}\left(\sigma^{-1} b\right)\left(X_{s}, \zeta_{s}\right)-\left(Z(\xi)_{s} \sigma^{-1} b\right)\left(X_{s}, \xi_{s}\right)\right)^{2} d s
\end{aligned}
$$

and

$$
d\left(Z(\zeta)_{t}^{-1}\right)^{2}=\left(Z(\zeta)_{t}^{-1}\right)^{2}\left(2\left(\sigma^{-1} b\right)\left(X_{t}, \zeta_{t}\right) d \widehat{B}_{t}+\left|\sigma^{-1} b\right|^{2}\left(X_{t}, \zeta_{t}\right) d t\right)
$$


Applying again the Itô formula,

$$
\begin{aligned}
& \frac{\left|Z(\zeta)_{t}-Z(\xi)_{t}\right|^{2}}{Z(\zeta)_{t}^{2}} \\
& =\int_{0}^{t} \frac{\left|Z(\zeta)_{s}-Z(\xi)_{s}\right|^{2}}{Z(\zeta)_{s}^{2}}\left(2\left(\sigma^{-1} b\right)\left(X_{s}, \zeta_{s}\right) d \widehat{B}_{s}+\left|\sigma^{-1} b\right|^{2}\left(X_{s}, \zeta_{s}\right) d s\right) \\
& \quad+2 \int_{0}^{t} \frac{\left(Z(\zeta)_{s}-Z(\xi)_{s}\right)}{Z(\zeta)_{s}^{2}}\left\{Z(\zeta)_{s}\left(\sigma^{-1} b\right)\left(X_{s}, \zeta_{s}\right)-Z(\xi)_{s}\left(\sigma^{-1} b\right)\left(X_{s}, \xi_{s}\right)\right\} d \widehat{B}_{s} \\
& \quad+2 \int_{0}^{t} \frac{\left(Z(\zeta)_{s}-Z(\xi)_{s}\right)}{Z(\zeta)_{s}^{2}}\left\{Z(\zeta)_{s}\left|\sigma^{-1} b\right|^{2}\left(X_{s}, \zeta_{s}\right)-Z(\xi)_{s}\left|\sigma^{-1} b\right|^{2}\left(X_{s}, \xi_{s}\right)\right\} d s \\
& \quad+\int_{0}^{t} \frac{1}{Z(\zeta)_{s}^{2}}\left|Z(\zeta)_{s}\left(\sigma^{-1} b\right)\left(X_{s}, \zeta_{s}\right)-\left(Z(\xi)_{s} \sigma^{-1} b\right)\left(X_{s}, \xi_{s}\right)\right|^{2} d s \\
& \quad+4 \int_{0}^{t} \frac{\left(Z(\zeta)_{s}-Z(\xi)_{s}\right)}{Z(\zeta)_{s}^{2}}\left(Z(\zeta)_{s}\left|\sigma^{-1} b\right|^{2}\left(X_{t}, \zeta_{s}\right)-Z(\xi)_{s}\left(\sigma^{-1} b\right)\left(X_{s}, \xi_{s}\right)\left(\sigma^{-1} b\right)\left(X_{s}, \zeta_{s}\right)\right) d s .
\end{aligned}
$$

$Z(\cdot)$ being an exponential martingale, the $L^{2}$-integrability of each integrands in the right-hand side of the preceding expression derive from the boundedness of $\sigma^{-1} b$. For any terms of the form

$$
\frac{1}{Z(\zeta)_{s}^{2}}\left\{Z(\zeta)_{s} g\left(X_{s}, \zeta_{s}\right)-Z(\xi)_{s} g\left(X_{s}, \xi_{s}\right)\right\}
$$

for $g$ equal to $\sigma^{-1} b$ or $\left|\sigma^{-1} b\right|^{2}$, we add and subtract the same element $Z(\zeta)_{s} g\left(X_{s}, \zeta_{s}\right)$ to get

$$
\frac{1}{Z(\zeta)_{s}}\left(g\left(X_{s}, \zeta_{s}\right)-g\left(X_{s}, \xi_{s}\right)\right)+\frac{1}{Z(\zeta)_{s}^{2}}\left(Z(\zeta)_{s}-Z(\zeta)_{s}\right) g\left(X_{s}, \xi_{s}\right)
$$

Noticing that $\sigma^{-1} b$ and $\left|\sigma^{-1} b\right|^{2}$ are bounded Lipschitz, by taking the expectation, and by introducing the appropriate pivots in the three last integrals, we get

$$
\begin{aligned}
\mathbb{E}_{\mathbb{Q}}\left[\frac{\left|Z(\zeta)_{t}-Z(\xi)_{t}\right|^{2}}{Z(\zeta)_{t}^{2}}\right] \leq & C\left\|\sigma^{-1} b\right\|_{\infty}^{2} \int_{0}^{t} \mathbb{E}_{\mathbb{Q}}\left[\frac{\left|Z(\zeta)_{s}-Z(\xi)_{s}\right|^{2}}{Z(\zeta)_{s}^{2}}\right] d s \\
& +C \min \left(\left\|\sigma^{-1} b\right\|_{L i p}, 2\left\|\sigma^{-1} b\right\|_{\infty}\right) \int_{0}^{t} \mathbb{E}_{\mathbb{Q}}\left[\frac{\left|Z(\zeta)_{s}-Z(\xi)_{s} \| \zeta_{s}-\xi_{s}\right|}{Z(\zeta)_{s}}\right] d s
\end{aligned}
$$

We end the proof of (ii) by applying Young's inequality.

\section{2 $\quad L^{2}$-existence and weak uniqueness}

$$
\left\{\begin{array}{l}
X_{t}=X_{0}+\int_{0}^{t} \sigma\left(X_{s}\right) d \widehat{B}_{s}, 0 \leq t \leq T \\
Y(\zeta)_{t}=Y_{0}+\int_{0}^{t} Z(\zeta)_{s} \mathbb{E}_{\mathbb{Q}}\left[Z(\zeta)_{s}^{-1} \ell\left(\zeta_{s}\right) \mid X_{s}\right] d s+\int_{0}^{t} Z(\zeta)_{s} \mathbb{E}_{\mathbb{Q}}\left[Z(\zeta)_{s}^{-1} \gamma\left(\zeta_{s}\right) \mid X_{s}\right] d W_{s}, \\
Z(\zeta)_{t}=\exp \left\{-\int_{0}^{t}\left(\sigma^{-1} b\right)\left(X_{s}, \zeta_{s}\right) d \widehat{B}_{s}+\frac{1}{2} \int_{0}^{t}\left|\sigma^{-1} b\right|^{2}\left(X_{s}, \zeta_{s}\right) d s\right\}, \\
\left(X_{0}, Y_{0}\right) \sim \mu_{0} .
\end{array}\right.
$$

Remark that, from (3.3), for any bounded Borel function $g: \mathbb{R}^{d} \rightarrow \mathbb{R}$,

$$
Z(\zeta)_{t} \mathbb{E}_{\mathbb{Q}}\left[\left(Z(\zeta)_{t}\right)^{-1}\left|g\left(\zeta_{t}\right)\right| \mid X_{t}\right]=\mathbb{E}_{\mathbb{P}}\left[\left|g\left(\zeta_{t}\right)\right| \mid X_{t}\right] \leq\|g\|_{L^{\infty}}, 0 \leq t \leq T,
$$

so that, $\left(Y(\zeta)_{t} ; 0 \leq t \leq T\right)$ is in $M^{2}(0, T)$. In addition, we have 
Proposition 3.5. There exists $0<C<\infty$ depending only on $T,\left\|\left(\sigma^{-1} b\right)\left(\sigma^{-1} b\right)^{*}\right\|_{L^{\infty}},\|\ell\|_{L^{\infty}}$ such that, for all $\zeta, \xi \in M^{2}(0, T)$, for all $0 \leq t \leq T$,

$$
\mathbb{E}_{\mathbb{Q}}\left[\left|Y(\zeta)_{t}-Y(\xi)_{t}\right|^{2}\right] \leq C \int_{0}^{t} \mathbb{E}_{\mathbb{Q}}\left[\frac{\left|Z(\zeta)_{s}-Z(\xi)_{s}\right|^{2}}{\left|Z(\xi)_{s}\right|^{2}}+\frac{\left|Z(\zeta)_{s}-Z(\xi)_{s}\right|^{2}}{\left|Z(\zeta)_{s}\right|^{2}}+\left|\zeta_{s}-\xi_{s}\right|^{2}\right] d s
$$

Proof. Applying Itô's formula, we get that

$$
\begin{aligned}
& \left|Y(\zeta)_{t}-Y(\xi)_{t}\right|^{2} \\
& =2 \int_{0}^{t}\left(Y(\zeta)_{s}-Y(\xi)_{s}\right)\left(Z(\zeta)_{s} \mathbb{E}_{\mathbb{Q}}\left[Z(\zeta)_{s}^{-1} \ell\left(\zeta_{s}\right) \mid X_{s}\right]-Z(\xi)_{s} \mathbb{E}_{\mathbb{Q}}\left[Z(\xi)_{s}^{-1} \ell\left(\xi_{s}\right) \mid X_{s}\right]\right) d s \\
& \quad+\int_{0}^{t}\left(Z(\zeta)_{s} \mathbb{E}_{\mathbb{Q}}\left[Z(\zeta)_{s}^{-1} \gamma\left(\zeta_{s}\right) \mid X_{s}\right]-Z(\xi)_{s} \mathbb{E}_{\mathbb{Q}}\left[Z(\xi)_{s}^{-1} \gamma\left(\xi_{s}\right) \mid X_{s}\right]\right) d W_{s} \\
& \quad+\int_{0}^{t} \operatorname{Trace}\left(\left(Z(\zeta)_{s} \mathbb{E}_{\mathbb{Q}}\left[Z(\zeta)_{s}^{-1} \gamma\left(\zeta_{s}\right) \mid X_{s}\right]-Z(\xi)_{s} \mathbb{E}_{\mathbb{Q}}\left[Z(\xi)_{s}^{-1} \gamma\left(\xi_{s}\right) \mid X_{s}\right]\right)\right. \\
& \left.\quad \times\left(Z(\zeta)_{s} \mathbb{E}_{\mathbb{Q}}\left[Z(\zeta)_{s}^{-1} \gamma\left(\zeta_{s}\right) \mid X_{s}\right]-Z(\xi)_{s} \mathbb{E}_{\mathbb{Q}}\left[Z(\xi)_{s}^{-1} \gamma\left(\xi_{s}\right) \mid X_{s}\right]\right)^{*}\right) d s .
\end{aligned}
$$

Taking the expectation on both sides of the preceding equality ( $L^{2}$ integrability is again ensured from the boundedness of the coefficients combined with Identity (3.3)), it follows

$$
\begin{aligned}
& \mathbb{E}_{\mathbb{Q}}\left[\left|Y(\zeta)_{t}-Y(\xi)_{t}\right|^{2}\right] \\
& =2 \int_{0}^{t} \mathbb{E}_{\mathbb{Q}}\left[\left(Y(\zeta)_{s}-Y(\xi)_{s}\right)\left(Z(\zeta)_{s} \mathbb{E}_{\mathbb{Q}}\left[Z(\zeta)_{s}^{-1} \ell\left(\zeta_{s}\right) \mid X_{s}\right]-Z(\xi)_{s} \mathbb{E}_{\mathbb{Q}}\left[Z(\xi)_{s}^{-1} \ell\left(\xi_{s}\right) \mid X_{s}\right]\right)\right] d s \\
& +\int_{0}^{t} \mathbb{E}_{\mathbb{Q}}\left[\operatorname { T r a c e } \left(\left(Z(\zeta)_{s} \mathbb{E}_{\mathbb{Q}}\left[Z(\zeta)_{s}^{-1} \gamma\left(\zeta_{s}\right) \mid X_{s}\right]-Z(\xi)_{s} \mathbb{E}_{\mathbb{Q}}\left[Z(\xi)_{s}^{-1} \gamma\left(\xi_{s}\right) \mid X_{s}\right]\right)\right.\right. \\
& \left.\left.\quad \times\left(Z(\zeta)_{s} \mathbb{E}_{\mathbb{Q}}\left[Z(\zeta)_{s}^{-1} \gamma\left(\zeta_{s}\right) \mid X_{s}\right]-Z(\xi)_{s} \mathbb{E}_{\mathbb{Q}}\left[Z(\xi)_{s}^{-1} \gamma\left(\xi_{s}\right) \mid X_{s}\right]\right)^{t}\right)\right] d s \\
& \quad
\end{aligned}
$$

By Young's inequality, for the first integral on the r.h.s., we have

$$
\begin{aligned}
& \int_{0}^{t} \mathbb{E}_{\mathbb{Q}}\left[\left(Y(\zeta)_{s}-Y(\xi)_{s}\right)\left(Z(\zeta)_{s} \mathbb{E}_{\mathbb{Q}}\left[Z(\zeta)_{s}^{-1} \ell\left(\zeta_{s}\right) \mid X_{s}\right]-Z(\xi)_{s} \mathbb{E}_{\mathbb{Q}}\left[Z(\xi)_{s}^{-1} \ell\left(\xi_{s}\right) \mid X_{s}\right]\right)\right] d s \\
& \leq \frac{1}{2} \int_{0}^{t} \mathbb{E}_{\mathbb{Q}}\left[\left|Y(\zeta)_{s}-Y(\xi)_{s}\right|^{2}\right] d s \\
& \quad+\frac{1}{2} \int_{0}^{t} \mathbb{E}_{\mathbb{Q}}\left[\left|Z(\zeta)_{s} \mathbb{E}_{\mathbb{Q}}\left[Z(\zeta)_{s}^{-1} \ell\left(\zeta_{s}\right) \mid X_{s}\right]-Z(\xi)_{s} \mathbb{E}_{\mathbb{Q}}\left[Z(\xi)_{s}^{-1} \ell\left(\xi_{s}\right) \mid X_{s}\right]\right|^{2}\right] d s
\end{aligned}
$$

In the last integral, adding and subtracting $Z(\xi)_{s} \mathbb{E}_{\mathbb{Q}}\left[Z(\zeta)_{s}^{-1} \ell\left(\zeta_{s}\right) \mid X_{s}\right]$ yields

$$
\begin{aligned}
& \mathbb{E}_{\mathbb{Q}}\left[\left|Z(\zeta)_{s} \mathbb{E}_{\mathbb{Q}}\left[Z(\zeta)_{s}^{-1} \ell\left(\zeta_{s}\right) \mid X_{s}\right]-Z(\xi)_{s} \mathbb{E}_{\mathbb{Q}}\left[Z(\xi)_{s}^{-1} \ell\left(\xi_{s}\right) \mid X_{s}\right]\right|^{2}\right] \\
& \leq 2 \mathbb{E}_{\mathbb{Q}}\left[\left(Z(\zeta)_{s}-Z(\xi)_{s}\right)^{2}\left(\mathbb{E}_{\mathbb{Q}}\left[Z(\zeta)_{s}^{-1} \ell\left(\zeta_{s}\right) \mid X_{s}\right]\right)^{2}\right]+2 \mathbb{E}_{\mathbb{Q}}\left[Z(\zeta)_{s}^{2}\left|\mathbb{E}_{\mathbb{Q}}\left[Z(\xi)_{s}^{-1} \ell\left(\xi_{s}\right)-Z(\zeta)_{s}^{-1} \ell\left(\zeta_{s}\right) \mid X_{s}\right]\right|^{2}\right] .
\end{aligned}
$$

The identity (3.3) then ensures that

$$
\begin{aligned}
\mathbb{E}_{\mathbb{Q}}\left[\left(Z(\zeta)_{s}-Z(\xi)_{s}\right)^{2}\left(\mathbb{E}_{\mathbb{Q}}\left[Z(\zeta)_{s}^{-1} \ell\left(\zeta_{s}\right) \mid X_{s}\right]\right)^{2}\right]= & \mathbb{E}_{\mathbb{Q}}\left[\frac{\left(Z(\zeta)_{s}-Z(\xi)_{s}\right)^{2}}{Z(\zeta)_{s}^{2}}\left(Z(\zeta)_{s} \mathbb{E}_{\mathbb{Q}}\left[Z(\zeta)_{s}^{-1} \ell\left(\zeta_{s}\right) \mid X_{s}\right]\right)^{2}\right] \\
& \leq\|\ell\|_{L \infty} \mathbb{E}_{\mathbb{Q}}\left[\frac{\left(Z(\zeta)_{s}-Z(\xi)_{s}\right)^{2}}{Z(\zeta)_{s}^{2}}\right]
\end{aligned}
$$


Using the properties of the conditional expectation, we also have

$$
\begin{aligned}
& \mathbb{E}_{\mathbb{Q}}\left[Z(\zeta)_{s}^{2}\left|\mathbb{E}_{\mathbb{Q}}\left[Z(\xi)_{s}^{-1} \ell\left(\xi_{s}\right)-Z(\zeta)_{s}^{-1} \ell\left(\zeta_{s}\right) \mid X_{s}\right]\right|^{2}\right] \\
& \leq \mathbb{E}_{\mathbb{Q}}\left[Z(\zeta)_{s}^{2} \mathbb{E}_{\mathbb{Q}}\left[\left|Z(\xi)_{s}^{-1} \ell\left(\xi_{s}\right)-Z(\zeta)_{s}^{-1} \ell\left(\zeta_{s}\right)\right|^{2} \mid X_{s}\right]\right] \\
& \leq 2\|\ell\|_{L^{\infty}}^{2} \mathbb{E}_{\mathbb{Q}}\left[Z(\zeta)_{s}^{2} \mathbb{E}_{\mathbb{Q}}\left[\left(Z(\xi)_{s}^{-1}-Z(\zeta)_{s}^{-1}\right)^{2} \mid X_{s}\right]\right] \\
& \quad+2 \mathbb{E}_{\mathbb{Q}}\left[Z(\zeta)_{s}^{2} \mathbb{E}_{\mathbb{Q}}\left[Z(\zeta)_{s}^{-2}\left|\ell\left(\xi_{s}\right)-\ell\left(\zeta_{s}\right)\right|^{2} \mid X_{s}\right]\right]
\end{aligned}
$$

where, by Lemma 3.4-(i),

$$
\begin{aligned}
\mathbb{E}_{\mathbb{Q}}\left[Z(\zeta)_{s}^{2} \mathbb{E}_{\mathbb{Q}}\left[\left(Z(\xi)_{s}^{-1}-Z(\zeta)_{s}^{-1}\right)^{2} \mid X_{s}\right]\right] & =\mathbb{E}_{\mathbb{Q}}\left[\mathbb{E}_{\mathbb{Q}}\left[Z(\zeta)_{s}^{2} \mid X_{s}\right]\left(Z(\xi)_{s}^{-1}-Z(\zeta)_{s}^{-1}\right)^{2}\left|\ell\left(\xi_{s}\right)\right|^{2}\right] \\
& =\mathbb{E}_{\mathbb{Q}}\left[\mathbb{E}_{\mathbb{Q}}\left[Z(\zeta)_{s}^{2} \mid X_{s}\right] \frac{\left(Z(\xi)_{s}-Z(\zeta)_{s}\right)^{2}}{Z(\xi)_{s}^{2} Z(\zeta)_{s}^{2}}\right] \\
& \leq C \mathbb{E}_{\mathbb{Q}}\left[\frac{\left|Z(\xi)_{s}-Z(\zeta)_{s}\right|^{2}}{Z(\xi)_{s}^{2}}\right]
\end{aligned}
$$

and

$$
\begin{aligned}
\mathbb{E}_{\mathbb{Q}}\left[Z(\xi)_{s}^{2} \mathbb{E}_{\mathbb{Q}}\left[Z(\xi)_{s}^{-2}\left|\ell\left(\xi_{s}\right)-\ell\left(\zeta_{s}\right)\right|^{2} \mid X_{s}\right]\right] & =\mathbb{E}_{\mathbb{Q}}\left[\mathbb{E}_{\mathbb{Q}}\left[Z(\xi)_{s}^{2} \mid X_{s}\right] Z(\xi)_{s}^{-2}\left|\ell\left(\xi_{s}\right)-\ell\left(\zeta_{s}\right)\right|^{2}\right] \\
& \leq C\|\ell\|_{\text {Lip }}^{2} \mathbb{E}_{\mathbb{Q}}\left[\left|\xi_{s}-\zeta_{s}\right|^{2}\right] .
\end{aligned}
$$

Putting the two last upper bounds together, we obtain the following bound for the 1.h.s of (3.10),

$$
\begin{aligned}
& \mathbb{E}_{\mathbb{Q}}\left[Z(\zeta)_{s}^{2}\left|\mathbb{E}_{\mathbb{Q}}\left[Z(\xi)_{s}^{-1} \ell\left(\xi_{s}\right)-Z(\zeta)_{s}^{-1} \ell\left(\zeta_{s}\right) \mid X_{s}\right]\right|^{2}\right] \\
& \leq C\|\ell\|_{L^{\infty}}^{2} \mathbb{E}_{\mathbb{Q}}\left[\frac{\left|Z(\xi)_{s}-Z(\zeta)_{s}\right|^{2}}{Z(\xi)_{s}^{2}}\right]+C\|\ell\|_{L i p}^{2} \mathbb{E}_{\mathbb{Q}}\left[\left|\xi_{s}-\zeta_{s}\right|^{2}\right] .
\end{aligned}
$$

For the second integral in (3.9), again by Young's inequality, we have

$$
\begin{aligned}
& \mathbb{E}_{\mathbb{Q}}[ \text { Trace }\left(\left(Z(\zeta)_{s} \mathbb{E}_{\mathbb{Q}}\left[Z(\zeta)_{s}^{-1} \gamma\left(\zeta_{s}\right) \mid X_{s}\right]-Z(\xi)_{s} \mathbb{E}_{\mathbb{Q}}\left[Z(\xi)_{s}^{-1} \gamma\left(\xi_{s}\right) \mid X_{s}\right]\right)\right. \\
&\left.\left.\times\left(Z(\zeta)_{s} \mathbb{E}_{\mathbb{Q}}\left[Z(\zeta)_{s}^{-1} \gamma\left(\zeta_{s}\right) \mid X_{s}\right]-Z(\xi)_{s} \mathbb{E}_{\mathbb{Q}}\left[Z(\xi)_{s}^{-1} \gamma\left(\xi_{s}\right) \mid X_{s}\right]\right)^{t}\right)\right] \\
&=\sum_{i, j, k=1}^{d} \mathbb{E}_{\mathbb{Q}}\left[\left(\left(Z(\zeta)_{s} \mathbb{E}_{\mathbb{Q}}\left[Z(\zeta)_{s}^{-1} \gamma^{i, j}\left(\zeta_{s}\right) \mid X_{s}\right]-Z(\xi)_{s} \mathbb{E}_{\mathbb{Q}}\left[Z(\xi)_{s}^{-1} \gamma^{i, j}\left(\xi_{s}\right) \mid X_{s}\right]\right)\right.\right. \\
&\left.\left.\quad \times\left(Z(\zeta)_{s} \mathbb{E}_{\mathbb{Q}}\left[Z(\zeta)_{s}^{-1} \gamma^{i, k}\left(\zeta_{s}\right) \mid X_{s}\right]-Z(\xi)_{s} \mathbb{E}_{\mathbb{Q}}\left[Z(\xi)_{s}^{-1} \gamma^{i, k}\left(\xi_{s}\right) \mid X_{s}\right]\right)\right]\right) \\
& \leq d \sum_{i, j=1}^{d} \mathbb{E}_{\mathbb{Q}}\left[\left|Z(\zeta)_{s} \mathbb{E}_{\mathbb{Q}}\left[Z(\zeta)_{s}^{-1} \gamma^{i, j}\left(\zeta_{s}\right) \mid X_{s}\right]-Z(\xi)_{s} \mathbb{E}_{\mathbb{Q}}\left[Z(\xi)_{s}^{-1} \gamma^{i, j}\left(\xi_{s}\right) \mid X_{s}\right]\right|^{2}\right] .
\end{aligned}
$$

Each component of the above sum can be bounded in the same manner than (3.11), replacing $\|\ell\|$ by some $\left\|\gamma^{i, j}\right\|$. Putting all together, we get, for some positive constant $C$,

$$
\mathbb{E}_{\mathbb{Q}}\left[\left|Y(\zeta)_{t}-Y(\xi)_{t}\right|^{2}\right] \leq C \int_{0}^{t} \mathbb{E}_{\mathbb{Q}}\left[\frac{\left|Z(\xi)_{s}-Z(\zeta)_{s}\right|^{2}}{Z(\xi)_{s}^{2}}+\frac{\left|Z(\xi)_{s}-Z(\zeta)_{s}\right|^{2}}{Z(\zeta)_{s}^{2}}\right] d s+C \int_{0}^{t} \mathbb{E}_{\mathbb{Q}}\left[\left|\xi_{s}-\zeta_{s}\right|^{2}\right] d s
$$


Combining the result of Proposition 3.5 with Lemma 3.4-(ii), and following the same procedure as for (3.1)-(3.2), we deduce with an appropriate choice of the constant $c$ that

$$
\|Y(\zeta)-Y(\xi)\|_{c}<\|\zeta-\xi\|_{c}, \forall \zeta, \xi \in M^{2}(0, T) .
$$

This ensures that the mapping $\zeta \in M^{2}(0, T) \mapsto Y(\zeta) \in M^{2}(0, T)$ which assigns to each element $\xi \in M^{2}(0, T)$, the solution $\left(Y(\zeta)_{t} ; 0 \leq t \leq T\right)$ given by (3.7) is contracting in $\left(M^{2}(0, T),\|\|_{c}\right)$. This enable us to conclude on the existence and uniqueness of a strong solution to (3.4).

By Girsanov transformation, this also enable us to conclude on the wellposedness of a weak solution to (1.2).

\subsection{Strong uniqueness}

The strong wellposedness of (1.2) will be given by a direct application of the following theorem due to Veretennikov [35]:

Theorem 3.6 (Theorem 1, [35]). Let $b:[0, \infty) \times \mathbb{R}^{d} \rightarrow \mathbb{R}^{d}$ be a bounded measurable function. Let $\sigma:[0, \infty) \times \mathbb{R}^{d} \rightarrow$ $\mathbb{R}^{d} \times \mathbb{R}^{d}$ be such that $a:(t, x) \mapsto a(t, x)=\sigma \sigma^{*}(t, x)$ is continuous, $x \mapsto a(t, x)$ is uniformly continuous in each compact $K \subset \mathbb{R}^{d}$, for any $t \in(0, T], 0<T<\infty$, and for some positive $\lambda$

$$
(\xi \cdot a(t, x) \xi) \geq \lambda|\xi|^{2}, \quad \text { for all } \xi \in \mathbb{R}^{d},(t, x) \in[0, \infty) \times \mathbb{R}^{d} .
$$

Moreover, assume that there exist some Borel functions $\sigma_{d} \in W_{\text {loc }}^{1,2 d+2}\left(\mathbb{R}^{n}\right), \sigma_{d+1} \in L_{\text {loc }}^{2 d+2}\left((0, \infty) ; W_{\text {loc }}^{1,2 d+2}\left(\mathbb{R}^{n}\right)\right)$ and $\sigma_{L}:(0, t) \times \mathbb{R}^{n} \times \mathbb{R}^{n} \rightarrow \mathbb{R}^{d} \times \mathbb{R}^{d}, \mathbb{R}^{n} \times \mathbb{R}^{n}$-Lipschitz continuous uniformly for $t \in(0, T], 0<T<\infty$, such that

$$
\sigma(t, x)=\sigma_{L}\left(t, \sigma_{d}(x), \sigma_{d+1}(t, x)\right)
$$

Then, given a $\mathbb{R}^{d}$-valued standard Brownian motion $\left(w_{t} ; t \geq 0\right)$, the stochastic differential equation:

$$
x_{t}=x+\int_{0}^{t} b\left(s, x_{s}\right) d s+\int_{0}^{t} \sigma\left(s, x_{s}\right) d w_{s}, \quad x \in \mathbb{R}^{d}, t \geq 0,
$$

has a unique strong solution.

We are going to show that the nonlinear diffusion coefficient

$$
(t, x) \mapsto \mathbb{E}_{\mathbb{P}}\left[\gamma\left(Y_{t}\right) \mid X_{t}=x\right]
$$

is continuous and admits a derivative (in the Sobolev sense) w.r.t. $x$ such that $\nabla_{x} \mathbb{E}_{\mathbb{P}}\left[\gamma\left(Y_{t}\right) \mid X_{t}=x\right]$ is locally in $L^{2 d+2}$-integrable on $(0, T) \times \mathbb{R}^{d}$. Before that, as a preliminary remark, let us point out that owing to (H0) and (H1), for all $t \geq 0$, the law of $X_{t}$ admits a density function $\rho_{X}(t, x)$. In addition, since $\rho_{X}(t, x)$ is a weak solution to

$$
\partial_{t} \rho_{X}+\nabla_{x}\left(\rho_{X} B_{\rho_{X}}\right)-\frac{1}{2} \operatorname{Trace}\left(\nabla_{x}^{2} \times\left(\rho_{X} \sigma \sigma^{*}\right)\right)=0,
$$

where $B_{\rho_{X}}=B_{\rho_{X}}(t, x)$ is the bounded Borel measurable $\mathbb{R}^{d}$-vector field given by

$$
B_{\rho_{X}}(t, x)=\mathbb{E}_{\mathbb{P}}\left[b\left(X_{t}, Y_{t}\right) \mid X_{t}=x\right],
$$

we have the following bounds (see e.g. Aronson [2])

$$
c \int G_{t}^{1 / \kappa}\left(x-x_{0}\right) \rho_{X}\left(0, x_{0}\right) d x_{0} \leq \rho_{X}(t, x) \leq C \int G_{t}^{\kappa}\left(x-x_{0}\right) \rho_{X}\left(0, x_{0}\right) d x_{0}, \quad x \in \mathbb{R}^{d}, 0 \leq t \leq T,
$$


where $G_{t}^{\kappa}$ is the centered Gaussian kernel with variance $\kappa^{2} t$ and $\kappa, c, C$ are some finite positive constants depending only on $T, d, a_{*}$ and $a *$. Then, under the assumption (H4), for all $0<R<\infty$, we have

$$
\begin{aligned}
\inf _{x \in B(0, R)} \rho_{X}(t, x) & \geq c \inf _{x \in B(0, R)} \int G_{t}^{1 / \kappa}\left(x-x_{0}\right) \rho_{X}\left(0, x_{0}\right) d x_{0} \\
& \geq c \inf _{x \in B(0, R)} \int G_{1}^{1 / \kappa}\left(x_{0}\right) \rho_{X}\left(0, x+t x_{0}\right) d x_{0} \\
& \geq c \inf _{x \in B(0, R)} \int_{B(0, R)} G_{1}^{1 / \kappa}\left(x_{0}\right) \rho_{X}\left(0, x+t x_{0}\right) d x_{0} \\
& \geq c \operatorname{sinf}_{x \in B(0, R), x_{0} \in B(0, R), 0 \leq t \leq T} \rho_{X}\left(0, x+t x_{0}\right)\left(\int_{B(0, R)} G_{1}^{1 / \kappa}\left(x_{0}\right) d x_{0}\right),
\end{aligned}
$$

that leads to the following lower bound for $\rho_{X}$ :

$$
\inf _{x \in B(0, R)} \rho_{X}(t, x) \geq m_{R}>0, \quad \text { with } \quad m_{R}:=c \inf _{z \in B(0, R+T R)} \rho_{X}(0, z)\left(\int_{B(0, R)} G_{1}^{1 / \kappa}\left(x_{0}\right) d x_{0}\right) .
$$

The positiveness of $\rho_{X}$ ensures that the component $\mathbb{E}_{\mathbb{P}}\left[\gamma\left(Y_{t}\right) \mid X_{t}=x\right]$ is defined a.e. on $(0, T) \times \mathbb{R}^{d}$ and writes as a Borel measurable function:

$$
(t, x) \mapsto \mathbb{E}_{\mathbb{P}}\left[\gamma\left(Y_{t}\right) \mid X_{t}=x\right]=\int \gamma(y) \mu^{Y \mid X=x}(t, d y, x), \text { for a.a. }(t, x) \in(0, T) \times \mathbb{R}^{d},
$$

where $\int \gamma(y) \mu^{Y \mid X=x}(t, d y, x)$ is the disintegration of $\int \gamma(y) \mu(t, d x, d y)$ for $\mu(t)=\operatorname{Law}\left(X_{t}, Y_{t}\right)$ w.r.t. $\rho_{X}(t, x) d x$. To exhibit the smoothness of $(t, x) \mapsto \mathbb{E}_{\mathbb{P}}\left[\gamma\left(Y_{t}\right) \mid X_{t}=x\right]$, we prove below a general result showing that any distribution of the form

$$
(t, x) \mapsto \int m(y) \mu(t, d x, d y), \quad \text { for any bounded Borel function } m: \mathbb{R}^{d} \rightarrow \mathbb{R},
$$

is absolutely continuous w.r.t. the Lebesgue measure on $\mathbb{R}^{d}$ and its related density is smooth in a suitable Sobolev sense. Such property is precisely given by the following lemma:

Lemma 3.7. Assume that Assumptions 3.1 and 3.2 hold. For $\left(X_{t}, Y_{t} ; 0 \leq t \leq T\right)$ the weak solution to (1.2), let $\mu(t)$ denote the joint law of $\left(X_{t}, Y_{t}\right)$ and let $\rho_{X}(t)$ be the density function of $\operatorname{Law}\left(X_{t}\right)$ for $0 \leq t \leq T$. Then, for any Borel measurable function $m: \mathbb{R}^{d} \rightarrow[0, \infty)$ not-identically equal to 0 , bounded, of class $\mathcal{C}^{2}\left(\mathbb{R}^{d}\right)$ on $\mathbb{R}^{d}$ such that its derivatives up to second order are bounded, the family of distributions

$$
\int m(y) \mu(t, d x, d y)
$$

admits a representant denoted $\overline{m \mu}$ in $L^{p}\left((0, T) ; W_{\text {loc }}^{2, p}\left(\mathbb{R}^{d}\right)\right)$ for any $p \geq d+2$.

Splitting $y \mapsto \gamma(y)$ into its positive part $(\gamma)^{+}$and negative part $(\gamma)^{-}$, Lemma 3.7 ensures that, $\mathbb{E}_{\mathbb{P}}\left[\gamma\left(Y_{t}\right) \mid X_{t}=x\right]$ rewrites according to

$$
\mathbb{E}_{\mathbb{P}}\left[\gamma\left(Y_{t}\right) \mid X_{t}=x\right]=\frac{\overline{\gamma \mu}(t, x)}{\rho_{X}(t, x)}\left(=\frac{\overline{(\gamma)^{+} \mu}(t, x)}{\rho_{X}(t, x)}+\frac{\overline{(\gamma)^{-} \mu}(t, x)}{\rho_{X}(t, x)}\right) .
$$

Since Lemma 3.7 also guarantees that $\rho_{X}$ and $\overline{\gamma \mu}$ are both in $L^{p}\left((0, T) ; W_{l o c}^{2, p}\left(\mathbb{R}^{d}\right)\right)$ for any $p$ large enough, the lower bound (3.13) further ensures that $(t, x) \mapsto \frac{\overline{\gamma \mu}(t, x)}{\rho_{X}(t, x)}$ is in $L^{p}\left((0, T) ; W_{l o c}^{2, p}\left(\mathbb{R}^{d}\right)\right)$. We then conclude on the strong uniqueness of $\left(X_{t}, Y_{t} ; 0 \leq t \leq T\right)$ solution to (1.2). 
Proof of Lemma 3.7. As a preliminary step, let us point out that, since $\rho_{X}(0)$ is in $L^{1}\left(\mathbb{R}^{d}\right) \cap L^{p}\left(\mathbb{R}^{d}\right)$ for $p \geq 2 d+2$, then $\rho_{X}(0) \in L^{r}\left(\mathbb{R}^{d}\right)$ for all $1 \leq r \leq p$. Combined with the Gaussian upper-bound in (3.12), this estimate ensures that, whenever $g: \mathbb{R}^{d} \mapsto \mathbb{R}$ is bounded, for all $0 \leq t \leq T$,

$$
\left|\int_{\mathbb{R}^{d}} \psi(x) \int_{\mathbb{R}^{d}} g(y) \mu(t, d x, d y)\right| \leq C\|g\|_{L^{\infty}\left(\mathbb{R}^{d}\right)}\|\psi\|_{L^{q}\left(\mathbb{R}^{d}\right)}, \quad 1 \leq \frac{q}{q-1} \leq 2 d+2, \psi \in \mathcal{C}_{c}^{\infty}\left(\mathbb{R}^{d}\right) .
$$

Riesz's representation theorem then implies that

$$
\int_{\mathbb{R}^{d}} g(y) \mu(t, d x, d y)=\overline{g \mu}(t, x) d x
$$

for some $\overline{g \mu}$ in $L^{\infty}\left((0, T) ; L^{r}\left(\mathbb{R}^{d}\right)\right)$.

By Itô formula, for all $\phi \in \mathcal{C}_{c}^{\infty}\left((0, T) \times \mathbb{R}^{d}\right)$, we have

$$
\begin{aligned}
& \mathbb{E}_{\mathbb{P}}\left[\int_{0}^{T} m\left(Y_{t}\right)\left(\partial_{t} \phi\left(t, X_{t}\right)+b\left(X_{t}, Y_{t}\right) \cdot \nabla_{x} \phi\left(t, X_{t}\right)+\frac{1}{2} \operatorname{Trace}\left(\sigma \sigma^{*}\left(X_{t}\right) \nabla_{x}^{2} \phi\left(t, X_{t}\right)\right)\right) d t\right] \\
& +\mathbb{E}_{\mathbb{P}}\left[\int_{0}^{T} \phi\left(t, X_{t}\right)\left(\mathbb{E}_{\mathbb{P}}\left[\ell\left(Y_{t}\right) \mid X_{t}\right] \cdot \nabla_{y} m\left(Y_{t}\right)+\frac{1}{2} \operatorname{Trace}\left(\nabla_{y}^{2} m\left(Y_{t}\right)\left(\mathbb{E}_{\mathbb{P}}\left[\gamma\left(Y_{t}\right) \mid X_{t}\right] \mathbb{E}_{\mathbb{P}}\left[\gamma^{*}\left(Y_{t}\right) \mid X_{t}\right]\right)\right)\right) d t\right]=0 .
\end{aligned}
$$

Rewriting the preceding expression into

$$
\begin{aligned}
& \int_{(0, T) \times \mathbb{R}^{2 d}} m(y)\left(\partial_{t} \phi(t, x)+b(x, y) \cdot \nabla_{x} \phi(t, x)+\frac{1}{2} \operatorname{Trace}\left(\sigma \sigma^{*}(x) \nabla_{x}^{2} \phi(t, x)\right)\right) \mu(t, d x, d y) d t \\
& +\int_{(0, T) \times \mathbb{R}^{2 d}} \phi(t, x)\left(\mathbb{E}_{\mathbb{P}}\left[\ell\left(Y_{t}\right) \mid X_{t}=x\right] \cdot \nabla_{y} m(y)\right) \mu(t, d x, d y) d t \\
& +\frac{1}{2} \int_{(0, T) \times \mathbb{R}^{2 d}} \phi(t, x) \operatorname{Trace}\left(\nabla_{y}^{2} m(y) \mathbb{E}_{\mathbb{P}}\left[\gamma\left(Y_{t}\right) \mid X_{t}=x\right] \mathbb{E}_{\mathbb{P}}\left[\gamma^{*}\left(Y_{t}\right) \mid X_{t}=x\right]\right) \mu(t, d x, d y) d t=0,
\end{aligned}
$$

we deduce that

$$
\overline{m \mu}(t, d x):=\int m(y) \mu(t, d x, d y)
$$

satisfies

$$
\begin{aligned}
& \int_{(0, T) \times \mathbb{R}^{d}}\left(\partial_{t} \phi(t, x)+\frac{1}{2} \operatorname{Trace}\left(\sigma \sigma^{*}(x) \nabla_{x}^{2} \phi(t, x)\right)\right) \overline{m \mu}(t, d x) d t \\
& +\int_{(0, T) \times \mathbb{R}^{d}}\left(\nabla_{x} \phi(t, x) \cdot \int m(y) b(x, y) \mu(t, d x, d y)\right) d t \\
& +\int_{(0, T) \times \mathbb{R}^{d}}\left(\phi(t, x) \mathbb{E}_{\mathbb{P}}\left[\ell\left(Y_{t}\right) \mid X_{t}=x\right] \cdot \int \nabla_{y} m(y) \mu(t, d x, d y)\right) d t \\
& +\frac{1}{2} \int_{(0, T) \times \mathbb{R}^{2 d}} \phi(t, x) \operatorname{Trace}\left(\mathbb{E}_{\mathbb{P}}\left[\gamma\left(Y_{t}\right) \mid X_{t}=x\right] \mathbb{E}_{\mathbb{P}}\left[\gamma^{*}\left(Y_{t}\right) \mid X_{t}=x\right] \nabla_{y}^{2} m(y)\right) \mu(t, d x, d y) d t=0 .
\end{aligned}
$$

Since $b, \ell, \gamma, m, \nabla_{y} m$ and $\nabla_{y}^{2} m$ are all bounded, we have

$$
\begin{aligned}
& \int_{(0, T) \times \mathbb{R}^{d}}\left(\partial_{t} \phi(t, x)+\frac{1}{2} \operatorname{Trace}\left(\sigma \sigma^{*}(x) \nabla_{x}^{2} \phi(t, x)\right)\right) \overline{m \mu}(t, d x) d t \\
& +\int_{(0, T) \times \mathbb{R}^{d}}\left(E_{1}[t, x]+E_{2}[t, x]\right) \phi(t, x) \overline{m \mu}(t, d x) d t \\
& =-\int_{(0, T) \times \mathbb{R}^{d}}\left(B[t, x] \cdot \nabla_{x} \phi(t, x)\right) \overline{m \mu}(t, d x) d t,
\end{aligned}
$$


where, following the convention in (3.14), $B[t, x]=\left(B^{(i)}[t, x] ; 1 \leq i \leq d\right), E_{1}[t, x]$ and $E_{1}[t, x]$ are given by

$$
\begin{aligned}
B^{(i)}[t, x] & =\frac{\overline{m b^{(i)} \mu}}{\overline{m \mu}}(t, x) \mathbb{1}_{\{\overline{m \mu}(t, x) \neq 0\}}, \\
E_{1}[t, x, \rho(t)] & =\sum_{i=1}^{d} \mathbb{E}_{\mathbb{P}}\left[\ell^{i}\left(Y_{t}\right) \mid X_{t}=x\right] \frac{\overline{\left(\partial_{y_{i}} m\right) \mu}}{\overline{m \mu}}(t, x) \mathbb{1}_{\{\overline{m \mu}(t, x) \neq 0\}}, \\
E_{2}[t, x] & =\sum_{i, j=1}^{d}\left(\mathbb{E}_{\mathbb{P}}\left[\gamma\left(Y_{t}\right) \mid X_{t}=x\right] \mathbb{E}_{\mathbb{P}}\left[\gamma^{*}\left(Y_{t}\right) \mid X_{t}=x\right]\right)^{i, j} \frac{\overline{\left(\partial_{y_{i} y_{j}}^{2} m\right) \mu}}{\overline{m \mu}}(t, x) \mathbb{1}_{\{\overline{m \mu}(t, x) \neq 0\}} .
\end{aligned}
$$

Since $m$ is not identically equal to 0 on $\mathbb{R}^{d}$, (3.12) implies that $\overline{m \mu}(t, x) \neq 0$ a.e. on $(0, T) \times \mathbb{R}^{d}$ and that the fraction in each of the above functions are defined a.e. on $(0, T) \times \mathbb{R}^{d}$.) Noticing that $B, E_{1}, E_{2}$ are all locally bounded, the continuity of $\overline{m \mu}$ on $(0, T) \times \mathbb{R}^{d}$ and its local Sobolev regularity then follow from the application of the following results from Bogachev et al. [3].

Proposition 3.8 (Corollaries 6.4.3 and 6.4.4 in [3]). Let $\mathcal{D} \subset \mathbb{R}^{d}$ be an open set. Assume that $a:(0, T) \times \mathbb{R}^{d} \rightarrow \mathbb{R}^{d \times d}$ is uniformly elliptic such that $a$ and $a^{-1}$ are locally bounded on $(0, T) \times \mathbb{R}^{d}$ and that, for $p>d+2$,

$$
\sup _{1 \leq i, j \leq d} \sup _{t}\left\|a^{i, j}\right\|_{W^{1, p}\left(B\left(x_{0}, R\right)\right)}<\infty \quad \text { for all } x_{0} \in \mathcal{D}, 0<R<\infty .
$$

Assume that $b^{1}, b^{2}, \cdots, b^{d}, f^{1}, f^{2}, \cdots, f^{d}$ are in $L_{\text {loc }}^{p}((0, T) \times \mathcal{D}), c$ is in $L_{\text {loc }}^{p / 2}((0, T) \times \mathcal{D})$, and assume that $\mu$ is a locally finite Borel measure on $(0, T) \times \mathbb{R}^{d}$ such that

$$
\int_{(0, T) \times \mathcal{D}}\left(\partial_{t} \phi+\operatorname{Trace}\left(a \nabla^{2} \phi\right)+b \cdot \nabla \phi+c \phi\right) \mu(d t, d x)=\int_{(0, T) \times \mathcal{D}} f \cdot \nabla \phi, \forall \psi \in \mathcal{C}_{c}^{\infty}((0, T) \times \mathcal{D}) .
$$

Then $\mu$ has a locally Hölder continuous density that belongs to the space $L^{p}\left(J ; W^{1, p}(V)\right)$ for all $J \subset(0, T), V \subset \mathcal{D}$ such that $J \times V$ has compact closure in $(0, T) \times \mathcal{D}$.

Acknowledgement: The second author has been supported by the Russian Academic Excellence Project "5-100".

\section{A Appendix}

The proof of the estimate (2.21) relies on the original arguments exhibited in [31] (pages 304-306) in a one-dimensional setting. We simply extend the result to a multidimensional case: For $\delta>0$, define

$$
\beta_{\delta}(t)=\mathbb{1}_{\{\delta \leq t \leq 1 / \delta\}}, G_{t}^{\gamma, \delta}(x, y)=\beta_{\delta}(t) G_{t}^{\gamma}(x, y) .
$$

Let $f$ be a $\mathcal{C}_{c}^{\infty}\left((0, T) \times \mathbb{R}^{d}\right)$-function so that $(t, x) \mapsto \int_{0}^{t} \triangle_{x} G_{t-s}^{\gamma}(f)(s, x) d s=\int_{0}^{t} \triangle_{x} G_{t-s}^{\gamma} * f(s, x) d s$ (for simplicity $G_{t}^{\gamma}$ denotes the $\mathcal{N}\left(0, \gamma^{2} t\right)$-Gaussian density function/kernel) is $\mathcal{C}^{\infty}\left((0, T) \times \mathbb{R}^{d}\right) \cap L^{2}\left((0, T) \times \mathbb{R}^{d}\right)$. By Parseval's equality: $\|h\|_{L^{2}\left(\mathbb{R}^{m}\right)}=\frac{1}{(2 \pi)^{m}}\|\mathcal{F}(h)\|_{L^{2}\left(\mathbb{R}^{m}\right)}, h \in L^{2}\left(\mathbb{R}^{m}\right), m \geq 1$, we get

$$
\left\|\int_{0} \triangle_{x} G_{.-s}^{\gamma, \delta} * f(s, x) d s\right\|_{L^{2}\left((0, T) \times \mathbb{R}^{d}\right)}=\frac{1}{(2 \pi)^{d+1}}\left\|\mathcal{F}\left(\triangle_{x} G^{\gamma, \delta}\right) \mathcal{F}\left(f \mathbb{1}_{\{[0, T]\}}\right)\right\|_{L^{2}\left(\mathbb{R}^{d+1}\right)}
$$

where $\mathcal{F}$ denote the Fourier transformation along the variables $t$ and $x$ :

$$
\mathcal{F}\left(f \mathbb{1}_{\{[0, T]\}}\right)(\tau, \xi)=\int_{\mathbb{R}} \int_{\mathbb{R}^{d}} \exp \{-\mathbf{i} t \tau-\mathbf{i} x \cdot \xi\} f(t, x) \mathbb{1}_{[0, T]} d t d x .
$$


Since

$$
\mathcal{F}\left(\triangle_{x} G^{\gamma, \delta}\right)(\tau, \xi)=-|\xi|^{2} \mathcal{F}\left(G^{\gamma, \delta}\right)(\tau, \xi)
$$

with

$$
\begin{aligned}
\mathcal{F}\left(G^{\gamma, \delta}\right)(\tau, \xi) & =\int_{0}^{T} \beta_{\delta}(t) \exp \{-\mathbf{i} t \tau\}\left(\int_{\mathbb{R}^{d}} \exp \{-\mathbf{i} \xi \cdot x\} G^{\gamma}(t, x) d x\right) d t \\
& =\int_{0}^{T} \beta_{\delta}(t) \exp \{-\mathbf{i} t \tau\} \exp \left\{-t \gamma^{2}|\xi|^{2} / 2\right\} d t \\
& =\frac{2}{2 \mathbf{i} \tau-\gamma^{2}|\xi|^{2}}\left(\exp \left\{-\delta\left(\mathbf{i} \tau-\gamma^{2}|\xi|^{2} / 2\right)\right\}-\exp \left\{-\frac{\left(\mathbf{i} \tau-\gamma^{2}|\xi|^{2} / 2\right)}{\delta}\right\}\right)
\end{aligned}
$$

we get

$$
|\xi|^{2}\left|\mathcal{F}\left(\triangle_{x} G^{\gamma, \delta}\right)(\tau, \xi) \mathcal{F}\left(f \mathbb{1}_{[0, T]}\right)(\tau, \xi)\right| \leq\left|\frac{-2|\xi|^{2}}{2 \mathbf{i} \tau-\gamma^{2}|\xi|^{2}}\right|\left|\mathcal{F}\left(f \mathbb{1}_{[0, T]}\right)\right|(\tau, \xi) \leq \frac{2}{\gamma^{2}}\left|\mathcal{F}\left(f \mathbb{1}_{[0, T]}\right)\right|(\tau, \xi) .
$$

Integrating both sides of the preceding inequality over $\mathbb{R}^{d+1}$, it follows that

$$
\begin{aligned}
\frac{1}{(2 \pi)^{d+1}}\left\|\int_{0} \mathcal{F}\left(\triangle_{x} G^{\gamma, \delta}\right) \mathcal{F}\left(f \mathbb{1}_{[0, T]}\right)\right\|_{L^{2}\left((0, T) \times \mathbb{R}^{d}\right)} & \leq \frac{2}{\gamma^{2}(2 \pi)^{d+1}}\left\|\mathcal{F}\left(f \mathbb{1}_{[0, T]}\right)\right\|_{L^{2}\left((0, T) \times \mathbb{R}^{d}\right)} \\
& \leq \frac{2}{\gamma^{2}}\|f\|_{L^{2}\left((0, T) \times \mathbb{R}^{d}\right)} .
\end{aligned}
$$

Since $\int_{0}^{\cdot} \triangle_{x} G_{.-s}^{\gamma}(f(s)) d s=\lim _{\delta \rightarrow 0^{+}} \int_{0}^{\cdot} \triangle_{x} G_{.-s}^{\gamma, \delta}(f(s)) d s$, we deduce, by extension, that

$$
\left\|\int_{0}^{\cdot} \triangle_{x} G_{.-s}^{\gamma}(f(s)) d s\right\|_{L^{2}\left((0, T) \times \mathbb{R}^{d}\right)} \leq \frac{2}{\gamma^{2}}\|f\|_{L^{2}\left((0, T) \times \mathbb{R}^{d}\right)}, \forall f \in \mathcal{C}_{c}^{\infty}\left((0, T) \times \mathbb{R}^{d}\right) .
$$

Since $\mathcal{C}_{c}^{\infty}\left((0, T) \times \mathbb{R}^{d}\right)$ is dense in $L^{2}\left((0, T) \times \mathbb{R}^{d}\right)$, we conclude (2.21).

In the same way, for any given positive definite $\mathbb{R}^{d \times d}$-matrix $\Gamma$ satisfying $\xi \cdot \Gamma \xi \geq \gamma^{2}|\xi|^{2}$ for some $\gamma \neq 0$, let $G_{t}^{\Gamma}$ be the $\mathcal{N}(0, \Gamma t)$-Gaussian density function and define, for $\beta_{\delta}$ as above,

$$
G_{t}^{\Gamma, \delta}=\beta_{\delta}(t) G_{t}^{\Gamma}
$$

Observing that

$$
\begin{aligned}
\left|\mathcal{F}\left(\partial_{x_{i} x_{j}}^{2} G^{\Gamma, \delta}\right)(\tau, \xi)\right| & =\left|\xi_{i} \xi_{j}\right|\left|\mathcal{F}\left(G^{\Gamma, \delta}\right)(\tau, \xi)\right| \\
& =\left|\xi_{i} \xi_{j}\right|\left|\int_{0}^{T} \beta_{\delta}(t) \exp \{-\mathbf{i} t \tau\} \exp \{-t(\xi \cdot \Gamma \xi) / 2\} d t\right| \leq \frac{2\left|\xi_{i} \xi_{j}\right|}{|2 \mathbf{i} \tau-(\xi \cdot \Gamma \xi)|} \leq \frac{2}{\gamma^{2}}
\end{aligned}
$$

then, for any family $F^{i, j} \in \mathcal{C}_{c}^{\infty}\left((0, T) \times \mathbb{R}^{d}\right), 1 \leq i, j \leq d$, we have

$$
|\xi|^{2}\left|\mathcal{F}\left(\partial_{x_{i} x_{j}}^{2} G^{\Gamma, \delta}\right)(\tau, \xi) \mathcal{F}\left(F^{i, j} \mathbb{1}_{[0, T]}\right)(\tau, \xi)\right| \leq \frac{2}{\gamma^{2}}\left|\mathcal{F}\left(F^{i, j} \mathbb{1}_{[0, T]}\right)\right|(\tau, \xi)
$$

from which we deduce, as previously, that

$$
\begin{aligned}
\left\|\sum_{i, j=1}^{d} \int_{0}^{\cdot} \partial_{x_{i} x_{j}}^{2} G_{.-s}^{\Gamma}\left(F^{i, j}(s)\right) d s\right\|_{L^{2}\left((0, T) \times \mathbb{R}^{d}\right)} & =\left\|\lim _{\delta \rightarrow 0^{+}} \sum_{i, j=1}^{d} \int_{0} \partial_{x_{i} x_{j}}^{2} G_{.-s}^{\Gamma, \delta}\left(F^{i, j}(s)\right) d s\right\|_{L^{2}\left((0, T) \times \mathbb{R}^{d}\right)} \\
& \leq \frac{2}{\gamma^{2}} \sum_{i, j=1}^{d}\left\|F^{i, j}\right\|_{L^{2}\left((0, T) \times \mathbb{R}^{d}\right)} .
\end{aligned}
$$




\section{References}

[1] Abergel, F. and Tachet, R., A nonlinear partial integro-differential equation from mathematical Finance, Discrete and Continuous Dynamical Systems - Series A (DCDS-A), 27(3): 907-917, 2010.

[2] Aronson, D. G., Bounds for the fundamental solution of a parabolic equation, Bull. Amer. Math. Soc., 73: 890-896, 1967.

[3] Bogachev, V. I., Krylov, N. V., Röckner, M., and Shaposhnikov, S., Fokker-Planck-Kolmogorov Equations, American Mathematical Society, 2015.

[4] Bossy, M., Jabir, J.-F. and Talay, D., On conditional McKean Lagrangian stochastic models. Probability theory and related fields, 151(1-2): 319-351, 2011.

[5] Bossy, M. and Jabir, J.-F., Lagrangian stochastic models with specular boundary condition. Journal of Functional Analysis, 268(6): 1309-1381, 2015.

[6] Bossy, M., Espina, J., Morice, J., Paris, C., and Rousseau, A., Modeling the wind circulation around mills with a Lagrangian stochastic approach, SMAI-Journal of Computational Mathematics, 2: 177-214, 2016.

[7] Bossy, M., Dupré, A., Drobinski, P., Violeau, L., and Briard, C., Stochastic Lagrangian approach for wind farm simulation, 2018. hal.inria.fr.

[8] Champagnat, N., and Jabin, P.-E., Strong solutions to stochastic differential equations with rough coefficients, To appear in Annals of Probability, 2018.

[9] Chorin A. J., Numerical study of slightly viscous flows, J. Fluid Mech., 57: 785-796, 1973.

[10] Durbin, P. A. and Speziale, C. G., Realizability of second moment closure via stochastic analysis, J. Fluid Mech. 280: 395-407, 1994.

[11] Evans, L. C., Partial Differential Equations, American Mathematical Institute, 1997.

[12] Figalli, A., Existence and uniqueness of martingale solutions for SDEs with rough or degenerate coefficients, Journal of Functional Analysis, 254(1): 109-153, 2008.

[13] Funaki, T., A certain class of diffusion processes associated with nonlinear parabolic equations, Z. Wahrsch. Verw. Gebiete, 67(3): 331-348, 1984.

[14] Guyon, J. and Henry-Labordère, P., The smile calibration problem solved, SSRN Electronic Journal, 2011.

[15] Gyöngy, I., Mimicking the one-dimensional marginal distributions of processes having an Itô Differential, Probability Theory and Related Fields, 71: 501-516, 1986.

[16] Jourdain, B. and Méléard, S., Propagation of chaos and fluctuations for a moderate model with smooth initial data, Ann. Inst. H. Poincaré Probab. Statist., 34(6): 726-766, 1998.

[17] Jourdain, B. and Reygner, J., Propagation of chaos for rank-based interacting diffusions and long time behaviour of a scalar quasilinear parabolic equation, Stoch. Partial Differ. Equ. Anal. Comput., 1(3): 455-506, 2013.

[18] Jourdain, B. and Zhou, A., Existence of a calibrated regime switching local volatility model and new fake Brownian motions, Preprint, 2016.

[19] Fournier, N., and Jourdain, B., Stochastic particle approximation of the Keller-Segel equation and twodimensional generalization of Bessel processes, The Annals of Applied Probability, 27(5): 2807-2861, 2017.

[20] Kohatsu-Higa, A. and Ogawa, S., Weak rate of convergence for an Euler scheme of nonlinear SDE's, Monte Carlo Methods and Applications, 3(4): 327-345, 1997. 
[21] Krylov, N. V., Lecture on Elliptic and Parabolic Equations in Sobolev Spaces, American Mathematical Society, 2008.

[22] Ladyženskaja, O. A., Solonnikov, V. A. and Uralćeva, N., Linear and Quasi-linear Equations of Parabolic Type, American Mathematical Society, coll. Translations of Mathematical Monographs, 1968.

[23] Lions, J.-L., Equations Différentielles Opérationnelles et Problèmes aux Limites, Grundlehren der mathematischen Wissenschaften, 1961.

[24] Méléard, S., Asymptotic behaviour of some interacting particle systems; McKean-Vlasov and Boltzmann models, Probabilistic models for nonlinear partial differential equations (Montecatini Terme, 1995), Lecture Notes in Math., 1627:42-95, 1996.

[25] Méléard, S., Monte-Carlo approximation for $2 d$ Navier-Stokes equations with measure initial data, Probab. Theory Relat. Fields 121: 367-388 (2001)

[26] Mishura, Y. S., and Veretennikov, A. Y., Existence and uniqueness theorems for solutions of McKean-Vlasov stochastic equations. Preprint, 2017.

[27] Oelschläger, K., A martingale approach to the law of large numbers for weakly interacting stochastic processes, The Annals of Probability, 12(2): 458-497, 1984.

[28] Oelschläger, K., A law of large numbers for moderately interacting diffusion processes, Zeitschrift für Wahrscheinlichkeitstheorie und verwandte Gebiete 69, no. 2: 279-322, 1985.

[29] Pope, S. B., Lagrangian pdf methods for turbulent flows, Annu. Rev. Fluid Mech., 26: 23-63, 1994.

[30] Pope, S. B., Turbulent Flows, Cambridge University Press, 11th edition, 2011.

[31] Stroock, D. and Varadhan, S. R., Multidimensional Diffusion Processes, Springer-Verlag, 1979.

[32] Sznitman, A. S., A propagation of chaos result for Burgers' equation, Probability Theory and Related Fields, 71, (4):581-613, 1986.

[33] Sznitman, A. S., Topics in Propagation of Chaos, École d'Été de Probabilités de Saint-Flour XIX—1989, p: 165-251, Lecture Notes in Math., 1464, Springer, 1989.

[34] Vasquez, J. L., The Porous Medium Equation, Oxford University Publications, 2006.

[35] Veretennikov, A., Yu., On strong solutions and explicit formulas for solutions of stochastic integral equations, Mat. Sb. (N.S.), 111(3): 434-452, 1980. 